\title{
Fabrication of resonant optical waveguide biosensors using electron gun depositions
}

\author{
Timothy Allen Cornell \\ West Virginia University
}

Follow this and additional works at: https://researchrepository.wvu.edu/etd

\section{Recommended Citation}

Cornell, Timothy Allen, "Fabrication of resonant optical waveguide biosensors using electron gun depositions" (2005). Graduate Theses, Dissertations, and Problem Reports. 1623.

https://researchrepository.wvu.edu/etd/1623

This Thesis is protected by copyright and/or related rights. It has been brought to you by the The Research Repository @ WVU with permission from the rights-holder(s). You are free to use this Thesis in any way that is permitted by the copyright and related rights legislation that applies to your use. For other uses you must obtain permission from the rights-holder(s) directly, unless additional rights are indicated by a Creative Commons license in the record and/ or on the work itself. This Thesis has been accepted for inclusion in WVU Graduate Theses, Dissertations, and Problem Reports collection by an authorized administrator of The Research Repository @ WVU. For more information, please contact researchrepository@mail.wvu.edu. 


\title{
FABRICATION OF RESONANT OPTICAL WAVEGUIDE BIOSENSORS USING ELECTRON GUN DEPOSITIONS
}

\author{
by \\ Timothy Allen Cornell \\ B.S.E.E, West Virginia University \\ Thesis submitted to the \\ College of Engineering and Mineral Resources at \\ WEST VIRGINIA UNIVERSITY \\ in partial fulfillment of the requirements for the degree of \\ Master of Science \\ in \\ Electrical Engineering
}

\section{Committee members:}

Dr. Dimitris Korakakis, Committee Chairperson

Dr. Lawrence A. Hornak

Dr. Mark Jerabek

Lane Department of Computer Science and Electrical Engineering

\section{Morgantown, West Virginia 2005}

Keywords: biosensors, electron beam deposition, FT-IR, ellipsometry 


\section{Abstract \\ Electron Beam Thin Film Depositions \\ Tim Cornell}

Biosensors are used to connect the chemical and physical worlds through the use of a transducer, allowing for quick detection of numerous biological agents. A new biosensor being developed, the resonant optical waveguide biosensor, uses power transfer between two optical waveguides to detect the presence of an antigen. The condition for power transfer is extremely sensitive to changes in index of refraction and allows the biosensor to have a high sensitivity to the antigen presence. Given the high sensitivity of the device, a high degree of accuracy in index of refraction and thickness is required from the fabrication process.

Fabrication of the biosensor transducer was performed from Electron Beam Physical Vapor Depositions. This thesis outlines the development of the protocol for the fabrication process and discusses the improvements made to the deposited films as new capabilities were added to the evaporation chamber such as substrate heat and Ion Beam Assisted Deposition (IBAD). Also discussed is the numerous characterization techniques used to optimize the fabrication protocol. 


\section{$\underline{\text { Table of Contents }}$}

Chapter One - Introduction

1.1) Biosensors 1
2
3
5

Chapter Two - Theory

2.1) Biosensors Operation 7

2.2) Vacuum Components 10

2.3) Vapor Depositions 14

2.4) Electron-Gun Physical Vapor Depositions 16

\begin{tabular}{ll}
$2.5)$ Ion Beam Assisted Depositions & 17 \\
\hline
\end{tabular}

\begin{tabular}{ll}
$2.6)$ Properties of Gases and Vapors & 21 \\
\hline 2.61$)$
\end{tabular}

2.6.1) Kinetic Theory of Gases and Vapors 22

2.6.2) Transport Theory 26

\begin{tabular}{ll}
$2.7)$ Surfaces & 27 \\
\hline 2.8$)$ & 29
\end{tabular}

2.8) Contamination and Substrate Cleaning 29

2.9) Characterization 31

2.9.1) Characterization using Fourier Transform Infrared Spectroscopy $\quad 31$

\begin{tabular}{ll} 
2.9.2) Characterization using Ellipsometry & 38 \\
\hline
\end{tabular}

Chapter Three - Experimental

3.1) Silicon Dioxide from Electron-Beam Depositions 4

3.1.1) Silicon Dioxide Depositions with Substrate Heat $\quad 53$

3.1.2) Silicon Dioxide Depositions with Ion Beam Assist $\quad 56$

\begin{tabular}{ll}
$3.1 .3)$ Silicon Dioxide Reactive Depositions & 60 \\
\hline
\end{tabular}

\begin{tabular}{ll} 
3.2) Sweep Pattern Selection & 61 \\
\hline 3.3$)$
\end{tabular}

3.3) Aluminum Oxide from Electron-Beam Depositions $\quad 65$

3.3.1) Aluminum Oxide Depositions with Substrate Heat 70

\begin{tabular}{ll} 
3.3.2) Aluminum Oxide Depositions with Ion Beam Assist & $\mathbf{7 3}$ \\
\hline
\end{tabular}

\begin{tabular}{ll} 
3.3.3) Aluminum Oxide Moisture Stability & 77 \\
\hline 3.4$)$
\end{tabular}

\begin{tabular}{lr}
$3.4)$ Structure Growth & 80 \\
\hline 3.41$)$
\end{tabular}

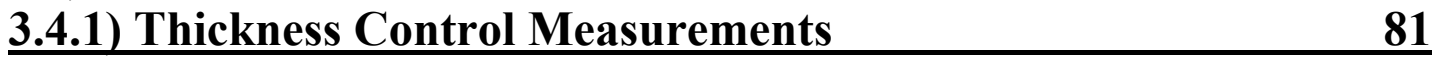

3.4.2) Ellipsometry, Prism Coupler, and Simulation Results $\quad 85$ 
Chapter Four - Conclusions

4.1) Improvements 90

4.2) Improved Thickness Control 90

4.3) Decreased Surface Roughness 91

4.4) Reactive Depositions of Aluminum Oxide 91

4.5) Ion Scrubbing, In-situ Surface Cleaning 91 


\section{Chapter One - Introduction}

\section{- Section 1.1}

\section{Biosensors}

The biosensor is a device that connects the biological world to the physical world [1]. Its three main components being the biological sensitive element (enzyme, antibody, etc.), the detector element which responds to changes in the biological element in some physical way (optical, electrical, magnetic, etc.), and the transducer in between which links the two. While many biological agents can be easily detected through more traditional means involving reagents, these processes are often time consuming and only possible in the laboratory environment. By including the receptor and the transducer into the biosensor device an analysis is completed quickly and can be performed in a variety of environments.

The traditional market for biosensors has been the detection of glucose for diabetes patients but is now finding a market in other fields [2]. They are currently in use for or being considered for the detection of a wide range of biological agents such as toxins, pathogens, and nutrients. The first commercial biosensor reached the market in 1975. Referred to as an amperometric biosensor it was used as a glucose analyzer by placing a silver anode and platinum cathode into a $\mathrm{KCl}$ and glucose solution. The reduction of oxygen from glucose oxidase at the cathode resulted in a current flow through the solution which was proportional to glucose concentrations. The same year biosensor research moved into the field of thermal biosensors which were used to measure the amount of heat generated by an exothermic reaction between an enzyme and a reactant. Also in 1975 biosensor research entered into the optical world with a fiber optic biosensor capable of detecting carbon dioxide or oxygen.

The resonant optical waveguide biosensor was first proposed by Don Lloyd and Harry Loats of Loats Associates Incorporated [3, 4]. This biosensor operates by sensing small changes in the index of refraction of the analyte located above two optical 
waveguides separated by a buffer layer of lower refractive index. An exponentially decaying electromagnetic wave, called an evanescent wave, which extends beyond the boundary of one waveguide and into the second waveguide allows for total power transfer back and forth between the two. This condition, known as coupled mode theory, is also very sensitive to the interaction of the evanescent wave with the layer above it, in this case the biolayer. The biosensor can be engineered so that this condition, known as resonance, only occurs when a specific biological agent has attached itself to the bioreceptor [3].

\section{- Section 1.2}

\section{Thin Film Fabrication}

A thin film is deposited onto a substrate when the properties required for a certain application are not obtainable from the thin film material in bulk.

\begin{tabular}{|l|l|}
\hline Property & Application \\
\hline Optical & $\begin{array}{l}\text { waveguides, antireflective } \\
\text { coatings }\end{array}$ \\
\hline Electrical & $\begin{array}{l}\text { insulating barriers, } \\
\text { semiconductors }\end{array}$ \\
\hline Mechanical & hardness, adhesion \\
\hline Chemical & $\begin{array}{l}\text { protective barriers against } \\
\text { oxidation of diffusion }\end{array}$ \\
\hline Magnetic & magnetic disks \\
\hline Thermal & heat sinks \\
\hline
\end{tabular}

Table 1.2.1 [5]

In addition, alternating layers of different thin film materials can often produce structures with completely new physical properties. Because of this, thin film processes have found their way into almost all manufacturing processes, from the automotive industry to cutting edge electrical and optical waveguide circuitry. 
In a thin film process, source material, which may be in a solid, liquid, vapor, or gas phase, is transported to the substrate where it attaches to the surface. For transport to take place from condensed source materials vaporization by heat or an energetic stream of electron, ions, or photons must occur. The properties of the transport phase can significantly affect the final properties of the thin film. If transport of the source material occurs through a high vacuum the material will arrive line-of-sight. This prevents oddly shaped substrate or side walls from photolithography processes from being covered and is often associated with physical vapor depositions. The final uniformity of the deposited film is dependent upon the geometry of the substrate holder and the chamber itself. Source material that is not transporting through a high vacuum (fluid-flow regime) will undergo many collisions with surrounding molecules before reaching the substrate. This allows for depositions on substrate with a variety of geometries. Thickness uniformity on the substrate is determined by gas flow patterns and diffusion.

Substrate conditions can result in serious modification of the thin film properties. Surface conditions (roughness, contamination, chemically active sites, crystallographic plane, etc.), reactivity of the transporting material, and additional energy input are the three principal factors of concern. Reactivity is dependent on the substrate and the material being deposited but is related to the "sticking coefficient" or the probability of a molecule that arrives at the substrate sticking to the surface and becoming part of the depositing film.

\section{- Section 1.2.1}

\section{Physical Vapor Depositions}

In Physical Vapor Depositions (PVD) the molecule sticks to the surface in a weakly bonded state known as physisorption. Numerous methods have been developed for performing these types of depositions.

Physical sputtering involves the acceleration of an ion towards a source target where several atomic collisions take place and one or more target atoms are ejected 
backwards from the target. A glow-discharge plasma is generated when a free electron is placed inside a gas and a electric field. The electron is accelerated through the gas due to the field and undergoes many collisions with gas molecules generating ions, and more electrons due to secondary electron emission. The resulting positive ions are accelerated away from the ion gun and towards the target where the targets atoms are sputtered.

Resistively heated thermal evaporators involve placing the source material into a small boat or crucible and passing a high current (several hundred amps) though it. Crucibles or boats are made of material with low electrical resistances and high melting temperatures such as tantalum, tungsten, or molybdenum and are good for thin film depositions when the material vaporizes at temperature below $1500^{\circ} \mathrm{C}$. Over time the molten material can weakly bond to the container or to an oxide layer formed on the container and the thermal expansions occurring during heat up and cool down may result in cracking of the container. As the container age's microcracks form and local hotspots occur resulting in vaporization of the container itself and contamination of the depositing film. Because of these limitations radiations shields are often used to prevent radiant heat loss and allow for depositions are lower power.

Radio frequency (rf) heated sources involve coupling rf energy into the source material or into a container holding the source material. This method has many of the same disadvantages as the resistively heat thermal evaporator when used with a conductive container but has been found to suffer less contamination when the energy is coupled directly into the source material.

Laser Ablation Deposition (LAD) involves using a high powered laser, usually a YAG or ArF, to deposit material in pulses. Using LAD high quality superconductors have been formed at low substrate temperatures. Depositions of patterns have also been achieved by shining the laser through a transparent substrate and vaporizing a film located near the substrates backside. LAD often produces vapor that is highly directions which makes it difficult to deposit on large areas. 
Electron beams are often required for the depositions of thin films from materials which vaporize above $1500^{\circ} \mathrm{C}$. In electron beam deposition a filament is held between $10-50 \mathrm{kV}$ below a copper hearth which contains the source material. A small potential is applied across the filament until enough current is passing through it so that electrons are thermionically emitted from it. The electrons are accelerated through the potential difference and steered towards the source material by magnetic deflection. The high energy electron bombardment can result in secondary electron emission from the source material leaving it with a positive charge after the electrons are quickly drawn towards ground. In dielectric substrates charge can build due to these positive ions and begin to repel ionized source material from the substrates surface. Also the high energy electrons can generate $\mathrm{x}$-rays when they strike the source material damaging the depositing films and substrates.

Chemical Vapor Depositions (CVD) occurring when the reaction of two products results in a deposited film and some byproduct such as the reaction of silane $\left(\mathrm{SH}_{4}\right)$ with oxygen to form silicon dioxide. An activation energy is required for the reactions to take place and can be added to the process in many different ways. Thermal CVD accomplishes this by raising the substrate temperatures to the required levels. A more common technique known as Plasma Enhanced Chemical Vapor Depositions (PECVD) overcomes the required activation energy through the use of charged particles from a glow-discharge plasma which allows for depositions at relatively low substrate temperatures while simultaneously allowing for ion bombardment for film structure improvements.

\section{- Section 1.2.2}

\section{Characterization Techniques}

The ability to accurately determine the chemical and physical properties of thin films is required for the optimization of the growth process. Thickness, refractive index, film intermix, surface roughness, stoichiometry, and material strain are common thin film characteristics of interest. 
IR spectroscopy uses the amount and location of absorption in the infrared spectrum to determine chemical bonding and molecular makeup in the films. Traditional methods involving the use of a monochromator and a slit to separate light from a broadband source are sometimes used but have mostly been replaced by interferometers in Fourier Transform Infrared Spectroscopy (FT-IR) which is capable of quicker analysis and higher spectral resolution.

The IR spectra of many thousands of different compounds are available in computer databases and IR data collection software can often rapidly identify compounds under investigation. If unable to make a positive match, the chemical bonds present can easily be extracted from a spectrum giving a good indication of the material present. This technique is particularly useful in vacuum systems where contamination control is critical. The IR spectrum of the thin film itself can be referenced out of the collected spectrum leaving only a spectrum of any contaminants that reside in the film.

Atomic Force Microscopy (AFM) is based on the forces experienced by a tip as it comes within a few angstroms of a surface. A tip of around $50 \mathrm{~nm}$ in radius is attracted by the van der Waals forces it experiences when it comes near or in contact with the film surface. Although the highest resolution images come from AFM when the tip comes into contact with the surface itself, it is sometimes necessary to restrict the amount of force on the surface for fear of damage. In tapping mode the AFM tip is vibrated above the surface of the sample, gently striking the surface each cycle but exerting less force on the surface than in contact mode. In non-contact mode the tip is again vibrated but is not allowed to come into contact with the film surface. While scanning the tip above the surface van der Waals forces change the amplitude of oscillation of the AFM tip.

Scanning Electron Microscopy (SEM) can be used to view a sample in an optical like manner but with the ability to resolve features that are much smaller than the wavelength of visible light. High energy electrons bombard the surface of the sample and secondary 
electrons are emitted. Secondary electron intensity and angle of emission are used to construct a visual image of the sample's surface morphology.

Ellipsometry is a powerful tool that allows for the determination thin film thickness, refractive index, and even microstructure. The focus of ellipsometry is to study the shape of elliptically polarized light that reflects off of a thin film or stack of thin films; or more specifically the ratio of amplitudes and phase difference between the parallel and perpendicular polarizations of the reflected light. By creating a general first estimate of the Fresnel reflection coefficients based on a model provided by the operator the ellipsometer can then slightly modify the operator's parameters to fit the model to the actual Fresnel reflection coefficients it has derived from the ratio and phase difference for the two polarizations. A more recent advance in ellipsometry techniques is the spectroscopic ellipsometer which collects data at many different wavelengths from the UV to the IR.

\section{Chapter Two - Theory}

\section{- Section 2.1}

\section{Biosensor Operation}

The resonant optical waveguide biosensor works by using two dielectric slab waveguides that are separated by a material of lower index all of which are deposited onto a substrate also of lower index.

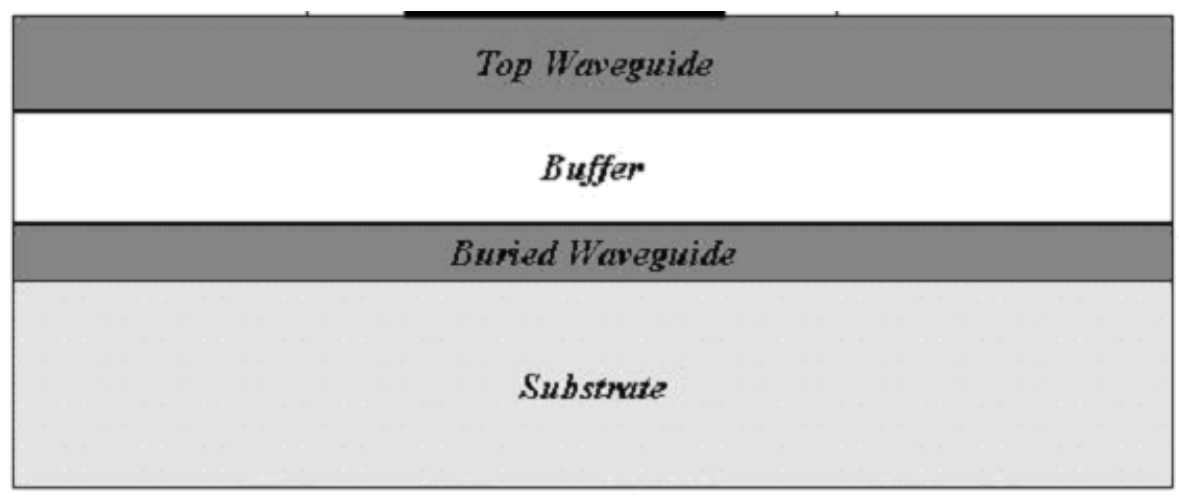

Figure 2.1.1 [6] 
For the resonant optical waveguide to work, light must propagate through the waveguide by total internal reflection. This occurs at all angles above what is known as the critical angle, which is given by

$$
\sin \theta_{c}=\frac{n_{2}}{n_{1}} \quad \text { Equation 2.1.1 }
$$

where

$\mathrm{n}_{1}=$ index of refraction of material light is traveling in

$\mathrm{n}_{2}=$ index of refraction of material just beyond the interface

If the material on either side of the slab waveguide is not of the same index then their will be a different critical angle for each reflection and the overall critical angle will be the greater of the two.

Although an electromagnetic wave will be totally reflected inside a waveguide when its incident angle is above the critical angle for both interfaces, due to lights wave nature the wave may not be sustainable. Electromagnetic waves can only propagate through a medium at certain angles known as "modes." At other angles the wave reflects off of one interface and overlaps with the incoming wave and destructively interferes. For the resonant cavity of a laser, the cavity length itself must be an integer multiple of one half the wavelength of the light inside the cavity

$$
L=\frac{m \lambda}{2} \quad \text { Equation 2.1.2 }
$$

For a standing wave to occur and the electromagnetic wave not to destructively interfere with itself. Optical waveguides are similar in that the phase shift of the wave in one complete round trip must be equal to an integer multiple of $2 \pi$.

$$
\Delta \phi=m 2 \pi \quad \text { Equation 2.1.3 }
$$




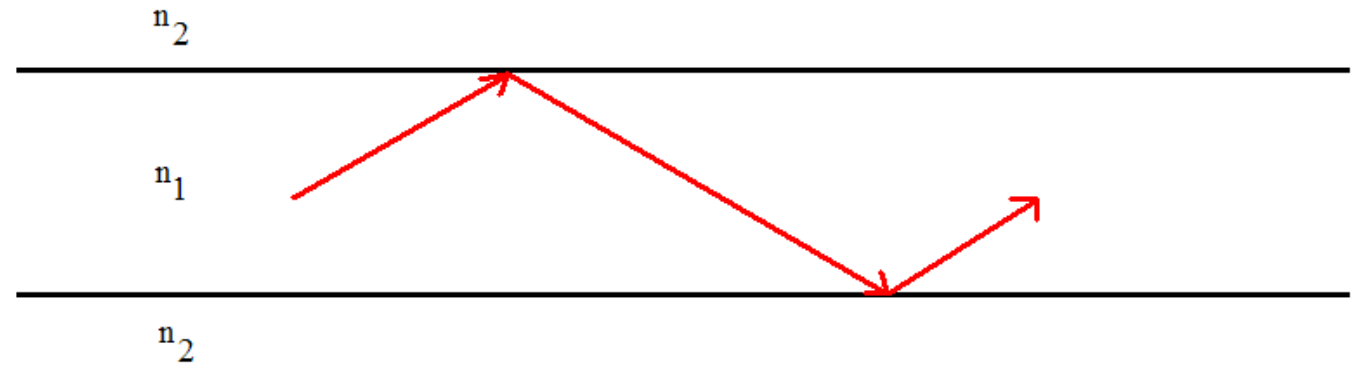

Figure 2.1.1

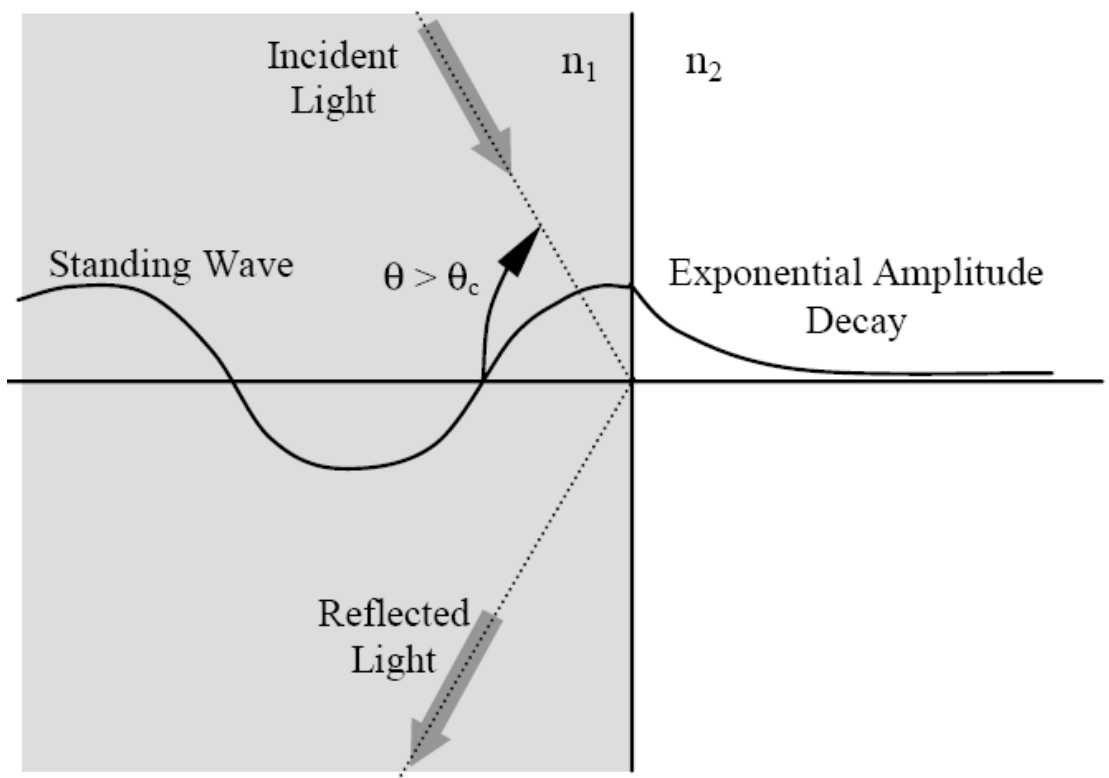

Figure 2.1.2 [7]

Even though at angles above the critical angle light is completely reflected from an interface, the wave traveling through a medium does not cease to exist beyond the interface. The exponentially decaying field extending beyond the interface is known as an evanescent wave and fades as

$$
e^{-\alpha z} \quad \text { Equation 2.1.4 }
$$

where

$$
\alpha=k_{0} \sqrt{n_{1}{ }^{2} \sin ^{2} \theta_{i}-n_{2}{ }^{2}} \quad \text { Equation 2.1.5 }
$$


The evanescent wave and Coupled Mode Theory are essential to the understanding of the resonant waveguide. If two dielectric waveguides are close enough together that the evanescent wave of one extends into the other, then Coupled Mode Theory finds that the electromagnetic wave can be transferred into the second waveguide after traveling some distance inside the first. The wave will then in turn travel some distance in the second waveguide before transferring back into the first waveguide. This resonant condition is very sensitive to the index of refraction of the medium just above the top waveguide which allows the sensor the ability to detect such small quantities of biological agents. This puts strict tolerances onto the biosensor manufacturing itself, because small changes in thicknesses or refractive indices could prevent the device from entering into its resonant condition when the agents to be detected are present.

\section{- Section 2.2}

\section{Vacuum Components}

Vacuum systems contain numerous components. For the greater than one percent in thickness accuracy that the biosensor requires it is imperative that all these components operate in a predictable and reproducible manner.

All vacuum systems consist of at least one type of pump. A mechanical pump, such as an oil sealed pump, dry pump, or a diaphragm pump, is often used to pump large amounts of gas out of a system. It is usually the first stage pump and is used to bring the chamber from atmosphere down to a specific pressure where a second pump will begin to operate. Sometimes referred to as positive displacement pumps, first stage pumps operate by capturing, compressing, and then expelling the gas collected [7].

Sorption pumps capture and hold gas molecules and are mostly used in high vacuum applications. A cryopump is type of sorption pump that uses progressively colder temperatures in the interior of the pump to trap gases, usually reaching temperatures down to $10-20$ Kelvin. If a gas solidifies at or above those temperatures it 
will form a solid and become trapped inside the pump. This is the case of many gases such as $\mathrm{O}_{2}, \mathrm{~N}_{2}, \mathrm{CO}_{2}$. For gases that do not solidify above the cryopumps operating temperatures it is still possible to trap the vapors by cryosorption. Even though the vapors may not condense, they can be cooled to such a degree that the molecules stick for a long periods of time when they reach the walls of the chamber. This process is exploited in the cryopump by a porous charcoal cylinder which provides greater surface area for the vapor molecules to stick to. Gases such as $\mathrm{H}_{2}, \mathrm{Ar}, \mathrm{Ne}$, and $\mathrm{He}$ are trapped in this manner.

Deposition of thin films with a high degree of accuracy would not be possible without some type of in-situ thickness monitoring. Optical measurements are extremely accurate ways to accomplish this but typically require a beam of light to enter into the chamber, reflect off the depositing film, and then exit the chamber to a detector. Optical measurements are also typically not accurate for films that are orders of magnitude in thickness above or below the wavelength of the light used. A more common way of insitu thickness monitoring is by monitoring the mass deposited on a small surface inside the chamber, often a small crystalline quartz wafer. Electrical contacts are made to the crystal and the deposition controller monitors the resonant frequency at which the quartz crystal is oscillating. As mass accumulates on the surface of the crystal the resonant frequency of the crystal will change, this can be modeled as a mass-spring oscillator.

$$
v_{r}=\frac{1}{T_{v}}=\frac{1}{2 \pi} \sqrt{\frac{k}{m}} \quad \text { Equation 2.2.1 }
$$

$v_{r}=$ resonant frequency, $\mathrm{Hz}$

$T_{v}=$ vibration period

$k=$ mass spring constant, $\mathrm{N} / \mathrm{m}\left(\mathrm{kg} / \mathrm{s}^{2}\right)$

$m=$ mass, $\mathrm{kg}$

and

$$
T_{v}=\sqrt{m_{q}}\left(1+\frac{1}{2} \frac{m_{f}}{m_{q}}\right) \text { Equation 2.2.2 }
$$


$m_{q}=$ initial mass of crystal

$m_{f}=$ final mass of crystal

This measurement requires a change from a mass measurement to a thickness. Therefore it is required that the density, $\mathrm{g} / \mathrm{cm}^{2}$, be known for the material being deposited. This can be a source of inaccuracy in the measurement if the density of the material is changing between depositions or even during deposition due to growth conditions. An account must also be made for the fact that it is not just the mass that is changing during deposition, the spring constant of the system overall is changing. This is observed as the acoustic impedance of the crystal/mass system and is known as the z-factor which is different from material to material. It must also be noted the quartz crystal will almost certainly be fixed to one position in the chamber and some correction factor must be determined to relate the thickness deposited at the stationary crystal to that of the rotating substrates.

The power of the crystal mass monitoring system is that it is a relative measurement, which in general is more accurate than absolute measurements. Common sources of inaccuracy in these measurements come from strain, which forms in the films, and from the temperature dependence of the quartz crystals. If strain is found in the films then quartz crystals will interpret this as a greater mass than is actually found on the crystal surface. A characteristic sign of this is a sudden negative shift in thickness when the stain is released in the films, often from cracking. Even greater sudden loss of thickness can be seen if the strain causes some of the film to crack and flake off of the crystal. For systems capable of monitoring from two different crystals located in the chamber at the same time it is possible to place crystals of different orientations inside the chamber. Growth on crystals of different orientation will often induce different amounts of strain in the films that will be detected as a difference in thickness.

Radiation due to multiple sources from inside the chamber can also affect the stability and accuracy of the thickness measurements. The greater the stability of the chamber temperature throughout the deposition process the greater the accuracy of the measurement overall can be considered. Water cooling is often added to crystal monitor 
heads to alleviate the sudden surge in radiation seen by the quartz crystal when the shutter is first open, often seen as a sudden drop into negative thicknesses. The crystal must absorb this heat and stabilize before accurate deposition rate can be achieved.

While crystal monitors are used mostly during the deposition process, a wealth of information can be obtained from them before and after the process itself. A sensitive measurement of sputtering rate can be determined from the crystals if an ion source is being used inside the chamber as long as care is taken to ensure that the monitor and electrical feedthroughs are correctly grounded. When a chamber is vented to atmosphere water may be absorbed into the films or oxidation may occur. In this case the crystal monitor can serve as a sensitive microbalance $( \pm 0.01 \mu \mathrm{g})$ for which porosity or water absorption can be determined.

Pressure gauges inside the chambers are primarily used to determine when one process can end and another can begin, (close roughing pump and open cryopump, begin deposition, etc.) but can also be used to determine pressure gradients inside the system if multiple gauges are present. Two simple pressure gauges that are often only used at low vacuum pressures are the thermocouple and Pirani gauge that are based on the removal of thermal energy by the gas still remaining inside the chamber. A voltage is placed across a simple coil and a thermocouple is attached. When negligible background gas is present inside the chamber a steady state is reached where energy lost from the heated coil is only from radiation. When enough background gases are present though they will conduct some heat away from the coil, which based on heat-transfer and fluid-flow models, can give the background pressures inside the chamber.

The ion gauge, which is often operated at pressures below $10^{-2}$ torr, uses an electron emitting filament, kept at positive potential, and a wire grid, kept at a higher positive potential, to determine background pressures. Electrons leave the filament due to field emission and are accelerated towards the wire grid due to the electric fields generated. Ionization of gas in the ion gauge creates an ionization current through the wire grid which is related to the emission current, ionization cross section, and gas 
concentration. The major drawback of the ion gauge is that different gases have different ionization cross sections and each ion gauge must be calibrated for the gas it will be used in. A ion gauge calibrated for air will not report a correct pressure reading in an oxygen rich atmosphere.

Shutters inside a vacuum chamber are placed to perform melt-ins of source material without depositing onto the substrates. A shutter is usually just a small flat disk or a cone shape if source material is diffusing around the shutter and making its way to the substrate.

Substrate heaters can be added to a vacuum chamber for chamber bake out and for substrate heating. Laser and electron emission can be used for heating but the most common technique is the use of radiation from a quartz lamp. Thermocouples are installed inside the chamber allowing for in-situ monitoring of the chamber temperature but are often only estimates as it is difficult to relate the temperature of the stationary thermocouple to the rotating substrates. Some materials, such as $\mathrm{SiO}_{2}$ are also very transparent in the IR which leads to further inaccuracies. Temperature sensitive tapes can be applied to the back of the substrates and are useful for determining the correlation between thermocouple temperature and substrate temperature.

\section{- Section 2.3}

\section{Vapor Depositions}

Vapor depositions can be spilt into two categories, Chemical Vapor Depositions (CVD) and Physical Vapor Depositions (PVD) [8]. In Chemical Vapor Deposition's gases are used as the source material rather than solid source materials. Condensation of the gases, and formation of the thin film, generally only occurs on surfaces that are above room temperature. Although a monolayer or two of gas may condense onto the chamber walls, condensation is generally restricted to the substrate which is often held at temperatures well above room temperature. The use of gases rather that solids as the 
source material and the ability to work at a variety or pressures allows operations in the two different process regimes, high-vacuum and fluid flow, where line of sight deposition is not required. The ability to operate in fluid-flow regime allows the gases to diffuse around oddly shaped geometries and condense on a variety of substrates, known as conformal coating, without the need to change sides of the substrate during deposition.

The classic chemical reactions that occur in CVD can be broken into five categories: pyrolysis, oxidation, hydrolysis, reduction, and displacement. In pyrolysis heat causes the deposition of one chemical compound into another such as in the reaction $\mathrm{SiH}_{4}(\mathrm{~g}) \rightarrow \mathrm{Si}(\mathrm{c})+2 \mathrm{H}_{2}(\mathrm{~g})$. Oxidation is the addition of oxygen or the removal of hydrogen from a substance, such as in the reaction

$\mathrm{SiH}_{4}(\mathrm{~g})+2 \mathrm{O}_{2}(\mathrm{~g}) \rightarrow \mathrm{SiO}_{2}(\mathrm{c})+\mathrm{H}_{2} \mathrm{O}(\mathrm{g})$. Hydrolysis is the process where a molecule is spilt into two by the addition of a water molecule, such as in the reaction $2 \mathrm{AlCl}_{3}(\mathrm{~g})+3 \mathrm{H}_{2} \mathrm{O} \rightarrow \mathrm{Al}_{2} \mathrm{O}_{3}(\mathrm{c})+6 \mathrm{HCl}(\mathrm{g})$. Reduction, the opposite of the oxidation reaction, is the removal of an oxygen or the addition of a hydrogen, such as in the reaction $\mathrm{WF}_{6}(\mathrm{~g})+3 \mathrm{H}_{2}(\mathrm{~g}) \rightarrow \mathrm{W}(\mathrm{c})+6 \mathrm{HF}(\mathrm{g})$. Finally, displacement is the reaction where one element replaces another in a compound or when the cations and anions of two compounds trade places, such as in the reaction

$\mathrm{Ga}\left(\mathrm{CH}_{3}\right)_{3}(\mathrm{~g})+\mathrm{AsH}_{3}(\mathrm{~g}) \rightarrow \mathrm{GaAs}(\mathrm{c})+3 \mathrm{CH}_{4}(\mathrm{~g})$. The main drawback of CVD can be seen in the reactions above; all of these reactions either use or produce as a byproduct a dangerous gas. A large segment of a CVD system will often be dedicated to safety, insuring that no leaks or valve failures occur.

The source gases used in CVD are generally not found in nature but are fabricated by reacting a desired element with a terminating radical, i.e. an atom or functional group with only one dangling bond. This fabrication often occurs at facilities other than the one where the CVD deposition will occur and the gas must be delivered to the facility. This fabrication and delivery increases the overall cost of CVD depositions as compared to PVD and provides a major entry route for contaminants. 


\section{- Section 2.4}

\section{Electron Gun Physical Vapor Depositions}

The basis of the electron beam gun is the electron emitting filament [9]. Electrons are thermoionicly emitted from a heated filament and accelerated through a $10 \mathrm{kV}$ voltage drop away from the filament. The electrons are then curled through an $180^{\circ}$ path rotation by a magnetic field which leads them towards the source material pockets. The combined force of electric and magnetic fields on the electron is given by

$$
\mathbf{F}=\mathbf{F}_{E}+\mathbf{F}_{B}=q_{e} \mathbf{E}+q_{e} \mathbf{v} \times \mathbf{B} \quad \text { Equation 2.4.1 }
$$

where $\mathbf{v}$ is the velocity of the electron given by

$$
v=\sqrt{\frac{2\left|q_{e}\right| V}{m_{e}}} \text { Equation 2.4.2 }
$$

$\mathrm{m}_{\mathrm{e}}$ is the mass of the electron, $9.11 \times 10^{-31} \mathrm{~kg}$ and $\mathrm{q}_{\mathrm{e}}$ the charge of the electron $-1.6 \times 10^{-19}$ C. The first force term in the equation, $\mathrm{q}_{\mathrm{e}} \mathbf{E}$, is the force that accelerates the electrons away from the filament towards the grounded source pockets, and is equal to the charge of the electron multiplied by the voltage drop between the gun and the pockets. The second is the force acquired by the electrons due to the large magnet inside the electron gun which produces a magnetic field perpendicular to the direction of the traveling electrons. The electrons are then curled due to the centrifugal force with a radius given by the cyclotron radius of an electron orbiting in a magnetic field which can be found by

$$
r_{c}=\frac{m_{e} v}{q_{e}|\mathbf{B}|} \quad \text { Equation 2.4.3 }
$$

where $v$ is the component of the electron velocity that is perpendicular to the magnetic field B. Using equation 2.4 .2 and an accelerating voltage of $10 \mathrm{kV}$ the velocity of an electron leaving the filament can be found to be $5.9 \times 10^{7} \mathrm{~m} / \mathrm{s}$. In a field of $10^{-2}$ webers $/ \mathrm{cm}^{2}$ (100 gauss) the radius of the electron path is found from equation 2.4.3 to be $3.4 \mathrm{~cm}$, a typical source to filament distance for an electron gun.

For all materials, at some point during the deposition, it is required to move the electron beam around the surface of the evaporant material. This is particularly true for 
materials such as $\mathrm{SiO}_{2}$ and $\mathrm{Al}_{2} \mathrm{O}_{3}$ because of their low thermal conductivity. If the beam is allowed to rest in one position it would quickly evaporate all the material in one location of the pocket and begin striking the bottom of the pocket. Scanning the beam is accomplished by two steering solenoid coils. The first coil, the longitudinal coil, produces a magnetic field in the same direction as the permanent magnet. This coil adds to the magnetic field and decreases the cyclotron radius which allows the electrons to be curled tighter and pushed towards the front of the pocket. The second coil, the latitudinal coil, produces a magnetic field that runs parallel to the plane of the copper hearth. This coil can produce a field pointing in either direction which creates a force pushing the electrons either to the left or to the right of the center of the evaporation source pockets.

In addition to the permanent magnet and the steering coils, four beam forming magnets are inside the electron gun. These magnets determine the size, shape, and intensity of the spot on the source pocket.

\section{- Section 2.5}

\section{Ion Assisted Depositions}

Ion Plating, or as it is more commonly referred to, Ion Assisted Deposition is the process of continually bombarding the depositing films with energetic particles, either ions or atoms. Advantages to this process are numerous, including in-situ substrate cleaning, increased adhesion, and modification of film properties such as index of refraction, film stress, and material stoichiometry [11,13]. Although the process that creates the thin films in a vacuum deposition can also be responsible for the creation of the energetic particles, more commonly ion generation is a separate process referred to as Ion Beam Assisted Deposition (IBAD).

For most ion sources the ionization process begins by the inducing highly energetic electrons into the gas to be ionized. For "hot cathode" sources the generation of electrons comes from the thermoelectron emitter, essentially the same as the electron-gun 
in an electron-beam evaporator. Current is passed through a filament until electrons traveling inside the filament gain enough thermal energy to break away from filament. This process is very popular in ion sources but has a major drawback that the ionization energies may drift as the ion source heats up. In contrast cold cathode ion sources operate without the need for a hot filament.

In cold cathode ion sources, gas enters into a compact chamber inside the ion source head where it becomes ionized due to a large electric field. The large applied voltage and small size of the ion source head force electrons to leave the cathode and travel through the ionized gas. The presence of a strong magnetic field inside the source head, due to the placement of several permanent magnets, prevents the electrons from traveling directly from the cathode to the anode. Due to the Hall Effect the electrons are instead steered in a circular fashion inside the source head. This increases the ionization by increasing collisions between electrons and gas molecules.

Ions leaving the ion source head are drawn towards the chambers walls which are held at ground. If non-conducting materials are being deposited inside the chamber electric charge will form on the inside of the chamber walls. The continuing bombardment of ions onto the substrate will result in charge build up and cause columbic repulsion of material from the substrate surface. To counteract this effect a hot filament is placed outside the ion source head which injects low energy electrons into the ion stream. These electrons do not neutralize the ions inside the beam but simply neutralize the ion stream as a whole leaving it a plasma.

When a plasma comes close to a substrate, a negative potential will form on the substrate. This potential, called the sheath potential, will form because of the more rapid loss of electrons from the plasma to the surface than the loss of ions. This potential will increase the force with which the ions strike the substrate surface and cause desorption of unwanted molecules from the surface, referred to as "ion scrubbing". If the energies of the impinging ions are great enough then they will be capable of physically sputtering the surface of the substrate. To increase momentum transfer between collisions and prevent 
any sorts of reactive processes this technique is usually only performed using large, inert atoms such as argon.

The improvements from IBAD result from the ability of the ions to transfer a portion of their momentum to the atoms or molecules on the surface of the depositing film. This energy transferred is referred to as the recoil energy as is given by the equation [12]

$$
T_{m}=\frac{4 m_{i} m_{t}}{\left(m_{i}+m_{t}\right)^{2}} E_{i} \quad \text { Equation 2.5.1 }
$$

$T_{m}=$ energy transferred

$m_{i}=$ impinging particle mass

$m_{t}=$ target particle mass

$E_{i}=$ initial energy of impinging particle

For an oxygen atom with a mass of $16 \mathrm{u}$ and an energy of $75 \mathrm{eV}$ which strikes an $\mathrm{Al}_{2} \mathrm{O}_{3}$ molecule with a mass of $101.9 \mathrm{u}$ the energy transferred is $35.19 \mathrm{eV}$. In contrast, if the impinging atom were an argon atom (mass equal to 40u) the energy transferred would have been $60.73 \mathrm{eV}$. While having the ion mass and the mass of the particles being deposited would produce the most efficient use of the ion source, one particle being within $50 \%$ mass of the other produces close to a $90 \%$ energy transfer which is acceptable in most instances. For this reason argon is one of the most common gases used ion sources, as it is the most common of the heavy inert gases. For processes that require reactive deposition, the use of the ion gas to form some other compound, a certain gas will be required and the reactive gas will be mixed with argon to increase the overall energy transfer between the ions and the surface particles.

Knowing the energy of the bombarding particles can give a great deal of insight into the deposition process. To that end, some ion sources will use a separate grid to steer ions of unwanted energies away from ion stream towards ground. Only ions of a certain energy or range of energies will be allowed to escape the ion source head. This design has the drawback of producing low ion current densities. Therefore most ion sources are of the "gridless" design where ions of a range of energies are produced. Unfortunately the spectrum of energies produced is not easily gathered and requires 
characterization to be performed on the system that the ion source is installed on. In addition, the energies of the ions that leave the ion source are really of no interest to the process engineer. The value of most concern is the energy with which the ion has when it reaches the sample surface. If the ion suffers any collisions during transport with background gases it will lose some energy. Energy loss will also occur if the ion becomes exposed to an electric field. This is very important with the deposition of dielectrics as the films often have some charge to them due to the electron beam deposition or from the ion stream itself.

When the ion arrives at the sample surface and collides with an absorbed particle that particle will gain a portion of the impinging particles' energy. The absorbed particle may now be freed from any intermolecular bonding that was occurring before and move around on the substrate. It may also desorb from the substrate and reabsorb at a different location on the substrate or it may leave the substrate completely. The ion source has given the atoms on the film's surface enough energy so that they may move around and fall into microscopic features such as pits or smooth out any bumps that have formed on the surface.

A high quality thin film should have a density close to that of the bulk material, low stress, and none of the gas used for the ion stream incorporated into the film. While adding IBAD to a deposition process has many benefits it is possible to increase the energy of the ions to a point where they begin to harm the overall deposition process. Bombardment of a thin film with overly energetic ions can increase the diffusion of the new material into the underlying film or substrate, reducing the abruptness of the interface. Bombarding ions will also heat the top few monolayers of the material increasing interdiffusion between an interface. While that may be advantageous in select instances, for optical coating where sharp transitions in the films are needed this harms the functionality of the device.

In addition to increasing the density of a film ion bombardment can also increase the compressive stress inside a film. If this stress increases beyond a critical point the 
films adhesion will decrease; cracks may form or the film may separate itself all together from other films or the substrate. Cracks and other problems can also occur if the ions become implanted inside the films. The gas used to form the ions will remain inside the films and increase the porosity of the films. The implanted gas may make its way outside of the film at a later time through the formation of a crack. This is particularly noticeable if the substrate temperature is kept to low; higher substrate temperatures will often allow any gas that sticks inside a pit in the depositing film to desorb from the film.

Contamination is another undesirable effect when performing IBAD [13]. The sputtering of material will not be restricted to only the substrate or the deposited films. Sputtering of the chamber walls often occurs and results in an increase of contaminants inside the chamber. Chamber wall conditioning with several nanometers of material before depositions begin is a common technique to prevent sputtering of contaminates from chamber walls.

\section{- Section 2.6}

\section{Properties of Gases and Vapors}

For a given volume and for a given temperature the pressure where as many molecules leave a condensed phase as return to the condensed phase is called the substances vapor pressure [5]. This is an important value in the theory of evaporation as it is related to the deposition rate of the process and whether the material is likely to sublime or form a liquid phase.

In practice a vapor pressure of $10^{-2}$ torr is required for a useful evaporation rate. Once this occurs the flux distribution of the vapor cloud can be modeled as a cosine distribution.

$$
\frac{d m}{d A}=\frac{E}{\pi r^{2}} \cos \phi \cos \theta \text { Equation 2.6.1 }
$$

$\mathrm{dm} / \mathrm{dA}=$ mass per unit area

$\mathrm{E}=$ total mass evaporated

$\mathrm{r}=$ distance from source to substrate 
$\Phi=$ angle from source - substrate line

$\Theta=$ angle from the normal to vaporizing surface

\section{- Section 2.6.1}

Kinetic Theory of Gases and Vapors

What occurs between the time the evaporate leaves the source and arrives at the surface of the substrate plays a major role the final characteristics on the deposited film. The status of the material and the events that occur to it during this period are referred to as the kinetic theory of gases.

The energy of a group of identical but distinguishable particles is modeled by the Maxwell-Boltzmann distribution as given by

$$
f(E)=e^{\left(\frac{-E}{k t}\right)} \text { Equation 2.6.2 }
$$

where $\mathrm{f}(\mathrm{E})$ is the probability of finding a particle at an energy $\mathrm{E}, \mathrm{T}$ is temperature, and $\mathrm{K}$ is Boltzmann's constant and is equal to $1.38 \times 10^{-23} \mathrm{~J} / \mathrm{K}$ or $8.62 \times 10^{-5} \mathrm{eV} / \mathrm{K}$. This energy distribution provides for maximum entropy for a closed system. The more energy a single particle obtains the fewer possible distributions there are for the remaining particles. Therefore probability decreases with increasing energy.

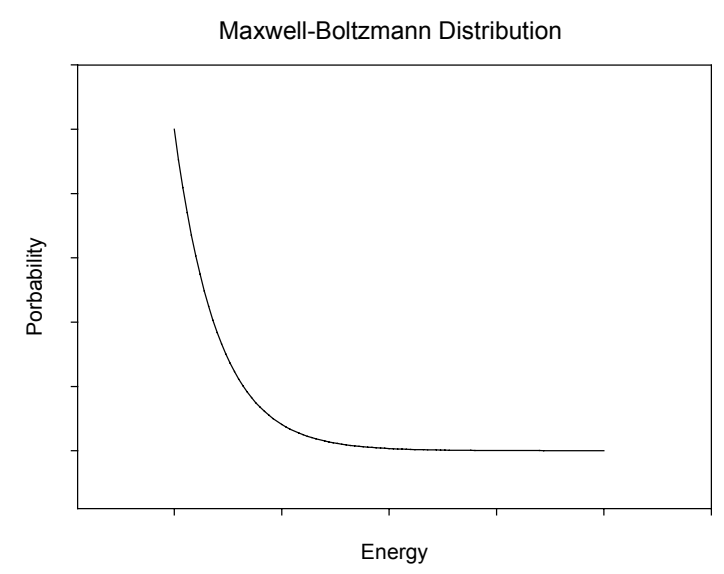

Figure 2.6.1 
The Maxwell-Boltzmann distribution becomes important in vapor depositions when some thermally activated process occurs [9]. In this case it is useful to determine the number of particles in the system that have an energy above the minimum energy required to activate the process, referred to as the activation energy and labeled $E_{a}$. First, the number of particles between energy $\mathrm{E}$ and $\mathrm{E}+\mathrm{dE}$ is known to be

$$
N f(E) \frac{d E}{k T} \quad \text { Equation 2.6.3 }
$$

where $\mathrm{N}$ is the total number of particle in the system. When integrating this between infinity and the activation energy the equation takes the form

$$
\int_{E_{a}}^{\infty} \exp \left[\frac{-E}{k T}\right] \frac{d E}{k T}=\exp \left[\frac{-E_{a}}{k T}\right] \quad \text { Equation 2.6.4 }
$$

which gives us the fraction of particles in the system at greater than or equal to $E_{a}$.

The velocity of the impinging particle is related to its energy and is important in determining the overall flux of the depositing vapor. The energy of a particle is related to velocity though translation kinetic energy which is the equation

$$
\begin{gathered}
E=\frac{m v^{2}}{2} \quad \text { Equation 2.6.5 } \\
\text { or } E=\frac{m\left(v_{x}{ }^{2}+v_{y}{ }^{2}+v_{z}{ }^{2}\right)}{2} \quad \text { Equation 2.6.6 }
\end{gathered}
$$

for three dimensional space. Using the Boltzmann distribution and assuming that the total energy found through it is related to translation energy (contributes to velocity) the probability of occupancy, which will be used to derive velocity, for a space $d_{x}, d_{y}, d_{z}$ is

$$
F\left(v_{x}, v_{y}, v_{z}\right)=\frac{\exp \left[-m\left(v_{x}{ }^{2}+v_{y}{ }^{2}+v_{z}{ }^{2}\right) / 2 k T\right]}{\int_{-\infty-\infty}^{\infty} \int_{-\infty}^{\infty} \int_{-\infty}^{\infty} \exp \left[-m\left(v_{x}{ }^{2}+v_{y}{ }^{2}+v_{z}{ }^{2}\right) / 2 k T\right] d v_{x} d v_{y} d v_{z}} \quad \text { Equation 2.6.7 }
$$

The numerator is simply the Boltzmann's distribution while 


$$
E=\frac{m\left(v_{x}^{2}+v_{y}^{2}+v_{z}^{2}\right)}{2} \quad \text { Equation 2.6.8 }
$$

the denominator is the normalization for the volume in question. The velocity in now derived from switching from Cartesian to polar coordinates and taking the limit as an infinitesimally small sphere, now

$$
F(v)=\frac{\exp \left[-m v^{2} / 2 k T\right]}{\int_{0}^{\infty} \int_{0}^{2 \pi} \int_{0}^{\pi} \exp \left[-m v^{2} / 2 k T\right] v^{2} \sin \theta d \theta d \varphi d v} \quad \text { Equation 2.6.9. }
$$

From these the most probably speed, the average speed, and the root-mean-square speed are found. The most probably speed occurs when the derivative of the velocity distribution is zero, which is

$$
v_{p}=\left(\frac{2 k T}{m}\right)^{1 / 2} \quad \text { Equation 2.6.10. }
$$

The average speed is found my integrating the probability and is equal to

$$
v_{a v}=\left(\frac{8 k T}{\pi m}\right)^{1 / 2} \quad \text { Equation 2.6.11 }
$$

and the rms found by the square root of the probability and is equal to

$$
v_{r m s}=\left(\frac{3 k T}{m}\right)^{1 / 2} \quad \text { Equation 2.6.12. }
$$

With these values the impingement rate, or the rate of collisions with the wall per unit area per unit time, can be calculated. Assuming the $x-z$ plane to be the wall the calculation only requires accounting for the y-component of the velocity. For a particle to strike the wall within a given time $\delta t$ and assuming a velocity $v_{y}$ the particle must be located within some area $v_{y} d t$ away from the wall. Integrating the velocity distribution over all $v_{x}$ and $v_{z}$ the expression becomes

$$
\left(\frac{m}{2 \pi k T}\right)^{1 / 2} \exp \left(\frac{-m v_{y}^{2}}{2 k T}\right) \quad \text { Equation 2.6.13. }
$$


Multiply this by the y-component of the velocity, the number of particles per volume (n), the time $\delta t$ and then integrating over the entire $\mathrm{v}_{\mathrm{y}}$ region the total number of particles striking the wall is

$$
N_{\text {total }}=n \delta t\left(\frac{k T}{2 \pi m}\right)^{1 / 2} \quad \text { Equation 2.6.14. }
$$

Dividing it now by time gives the impingement rate

$$
z=n\left(\frac{k T}{2 \pi m}\right)^{1 / 2} \quad \text { Equation 2.6.15 }
$$

and in polar form

$$
j_{\Omega}(\theta, \varphi)=\int_{0}^{\infty} n \cdot v \cos \theta \cdot v^{2} F(v) d v=\frac{n \cos \theta v_{a v}}{4 \pi} \quad \text { Equation 2.6.16. }
$$

The ideal gas law is the most useful tool available for theoretically determining the pressure based on the kinetic theory of gases. The pressure is due solely to the transfer of momentum from the particles to the chamber walls. The value is derived from taking the particles with velocity between $\mathrm{v}_{\mathrm{y}}$ and $\mathrm{v}_{\mathrm{y}}+\mathrm{dv}_{\mathrm{y}}$ times the momentum transfer per collision per unit time

$$
d P\left(v_{y}\right)=\frac{n F_{y} d v_{y} \cdot v_{y} \delta t \cdot 2 m v_{y}}{\delta t} \quad \text { Equation 2.6.17 }
$$

and total pressure equals

$$
P=n k T \quad \text { Equation 2.6.18 }
$$

Mean free path of a particle inside a vacuum chamber is the distance on average that the particle will travel before it collides with another particle. This value has significant importance in film formation, especially along the impinging direction. The size of the particles under consideration plays a significant role in the calculation, the size of interest here is the collision cross section and is simply the diameter of a sphere, $\pi d^{2}$. The volume that the particle sweeps through is at a rate of $\pi d^{2} v_{a v}$ and encounters other particles at a rate $\pi d^{2} v_{a v} n$. Taking into account that the particles have a relative speed to one another the collision rate is given by

$$
r=\sqrt{2} \pi d^{2} v_{a v} n \quad \text { Equation 2.6.19. }
$$


and the mean free path is simply the inverse times the average velocity

$$
\lambda=\frac{1}{\sqrt{2} \pi d^{2} n} \quad \text { Equation 2.6.21. }
$$

A more useful form of this equation allows the determination of the mean free path of an impurity gas inside a host gas. For this the mean free path is

$$
\lambda_{2}=\frac{1}{\pi\left(d_{21}\right)^{2}}\left[\frac{1+\left(v_{1 a v}\right)^{2}}{\left(v_{2 a v}\right)^{2}}\right]^{1 / 2} n_{1} \quad \text { Equation 2.6.22 }
$$

where the impurity is particle 2 and the host gas is particle 1 . The relative velocity for the gas is now

$$
v_{r e l}=\left[\left(v_{2 a v}\right)^{2}+\left(v_{1 a v}\right)^{2}\right]^{1 / 2} \quad \text { Equation 2.6.23 }
$$

and the average collision cross section is now

$$
d_{21}=\frac{d_{2}+d_{1}}{2} \text { Equation 2.6.24. }
$$

\section{- Section 2.6.2}

\section{Transport Theory}

In a PVD process the material leaving the source often has a mean free path that is much larger than the distance from the source to the substrate [8]. This means that the material will often reach the substrate line of sight and no collisions with the chambers' contaminant gas molecules will occur. Determination of the mean free path is of such importance in vacuum systems because this establishes the behavior of the molecules inside the chamber. Inside the chamber the evaporated material can either behave as a high vacuum process or a fluid-flow process. Determination of this is based upon the value known as the Knudsen number which is given by the ratio of the mean free path to source to substrate distance

$$
K_{n}=\frac{l}{L} \quad \text { Equation 2.6.27 }
$$

where $l$ is the mean free path and $\mathrm{L}$ is the distance between the substrate and the source material. For values of $\mathrm{K}_{\mathrm{n}}>1$ the process is considered high vacuum and the molecules 
or atoms will flow independent of each other. For $\mathrm{K}_{\mathrm{n}}<0.01$ the process is a fluid-flow process. For $\mathrm{K}_{\mathrm{n}}$ values in between, the specific activities of the vapor cloud inside the chamber are complicated to determine.

\section{- $\quad$ Section 2.7}

\section{Surfaces}

A thin films' properties have a strong correlation to the properties of the substrate below it; a rough substrate will produce a rough film surface, more activation sites will increase adhesion to the substrate, etc. Therefore a reproducible film requires a reproducible substrate [8]. Many different types of substrate are available, metals are used when an electrically conductive substrate is needed or when an easily machined material is required. All metals except for gold form some degree of a native oxide on their surface which can affect film growth. Due to the high surface energy of these oxides other particles will often be absorbed to lower the surface energy. This is a significant source for contaminants when using metal substrates. Ceramics and glasses are used when a substrate with a low thermal and electrical conductivity is required or when the optical properties of the substrate are of concern. Polymers can also be used for substrates. Polymers are essentially long chains of smaller groups known are monomers which are usually made of carbon and hydrogen. Bond lengths, orientations, and strengths can all be varied in polymers to produce a wide range of substrate characteristics.

Modification of a substrates' surface occurs before a deposition to optimize the surface conditions for the deposition. Outgassing of a substrate arises when a species inside the material moves from the interior to the surface and then vaporizes. Many atmospheric gases or solvents that were used to clean the substrate are often found inside the pores of the substrate and are often the most significant component of the outgassing. If these vapors become trapped inside the substrate due to the depositing films, cracks or bubbles may occur when the gases are forced out of the substrate for some reason, such as annealing. This undesirable effect can be avoided by making sure all outgassing species have left the substrate before deposition. This is accomplished by a sensitive 
weight-loss measurement, mass spectrometer, or IR absorption spectrum taken through the chamber.

Outdiffusion of a material occurs when a group leaves the bulk and reaches the surface but does not vaporize, which occurs to stainless steel at high temperatures. This outdiffused material is now considered a contaminant on the surface of the substrate. Measurement of outdiffusion is much more difficult to measure than outgassing and often requires some chemical analysis of the surface.

A smoother substrate surface will produce a smoother deposited film and steps to smooth the substrate before deposition are often performed. Mechanical polishing can be used when the degree of smoothness is not beyond the micron scale but often damages the top few monolayers of a crystalline surface. For even smoother surfaces chemical etching can be performed to preferentially remove the high points on the surface.

Roughening a substrate surface can be performed to increase adhesion of the depositing film to the surface. The depositing particles fill in the holes on the substrate creating an intermix or transition layer between the two materials. Roughening can be accomplished by mechanical polishing with a higher grit size or by blasting the substrate with an abrasive or by chemical means. Chemically etching occurs more rapidly at preferential sites such as grain boundaries, certain crystal facets, or other defects. For many metals, surface roughening can occur by intentionally oxidizing the surface and then etching the oxide, leaving the underlying roughened metal surface.

Surface activation is the process of increasing the chemical reactivity of the surface to increase the adhesion of the depositing film. For polymers, an oxygen plasma above the surface of the substrate can increase adhesion due to the formation of the acidic $\mathrm{C}=\mathrm{O}$ groups. For nonconducting materials such as glasses and ceramics increased surface activation can occur when electronic charges sites are generated. The use of radiation or bombardment of a charged particle can leave the substrate surface with some electrical potential; oppositely charged depositing molecules will be attracted to the 
surface increasing the likelihood of bonding. Ion bombardment further increases surface activation by increasing surface roughness on the nanometer scale by sputtering material from the substrate surface.

\section{- Section 2.8}

\section{Contamination and Substrate Cleaning}

A contaminate is any particle that interferences with the depositing thin film formation, properties, or stability in a negative away. Removal of these contaminate is an integral part of the thin film deposition process. A contaminate may cover the entire substrate surface, such as the formation of an oxide layer or the adsorption of hydrocarbons, or it may only cover a small area as with dust particles or fingerprints. Cleaning is the removal of these surfaces contaminates and is divided into to categories: ex situ which occurs before placement in the vacuum chamber and in situ which occurs inside the vacuum chamber.

A variety of ex situ cleaning methods are available, the method chosen depends on the substrate and its ability to withstand the process itself. Removal of gross contaminates from the substrate surface can be accomplished by abrasive cleaning by blasting the surface with glass beads or by polishing the surface with a very rough material such as steel wool or sand paper. These methods can leave a previously polished surface extremely rough and implant the abrasive material into the substrate.

Chemical etching can remove surface contaminates from a substrate in a very selective manner. Acid baths are used for metals to remove the native oxide that naturally occurs on their surface but have the negative effect of introducing hydrogen atoms into the surface causing it to become brittle or leaving the surface hydrogen terminated, and hydrophilic, or hydroxyl terminated and hydrophobic. Solvents are generally less aggressive than acids are used to dissolve the contaminate into its solution. Often a cleaning solution will consist of a mixture of polar solvents, which dissolve polar contaminates such as ionic salts, or nonpolar solvents, which dissolve nonpolar 
contaminates such as oil. Many of these solvents themselves also leave a contaminate film on the surface which must also be removed by either a different solvent or by heating the substrate and evaporating the solvent from the substrate.

In situ cleaning is intended to remove the contaminates that have arrived at the substrate surface between the time the substrate left its ex situ cleaning and arrived into the substrate chamber. Plasma cleaning of a substrate involves cleaning with a reactive gas, such as oxygen or hydrogen, to produce compounds which can be more easily removed by the vacuum chamber's pump, i.e. hydrocarbons to $\mathrm{CO}$ or $\mathrm{CO}_{2}$.

Ion scrubbing involves bombarding the substrate surface with inert energetic ions. A momentum transfer occurs between the ions and contaminants, desorbing them from the surface. When this process does not provide the energy needed to remove the contaminants then physical sputtering of the surface can be used. This process involves removing the surface layer of the substrate along with the contaminates. Because of this, sputtering is regarded as a universal contaminant remover; however some particles such as hydrocarbons can me more difficult to remove due to their shape. When sputtering it is possible the gas used can react with the substrate or become implanted into the substrate. It is common to see the substrate heated during sputtering in an attempt to outgas the sputtering gas and any other gas that has incorporated itself into the substrate before being loaded into the vacuum chamber.

Once the film has been removed from the vacuum chamber it is more susceptible to contamination than the substrate was. Deposited films will often have more surface area due to porosity and columnar film morphology and more area for contaminants to become lodged. Almost all materials will react in some way with the atmosphere when brought out of the vacuum chamber and the film surface will be changed. A common example is the formation of a native oxide on deposited metals. Many evaporation systems are designed with numerous source pockets so multiple films can be deposited without the need to break vacuum. 


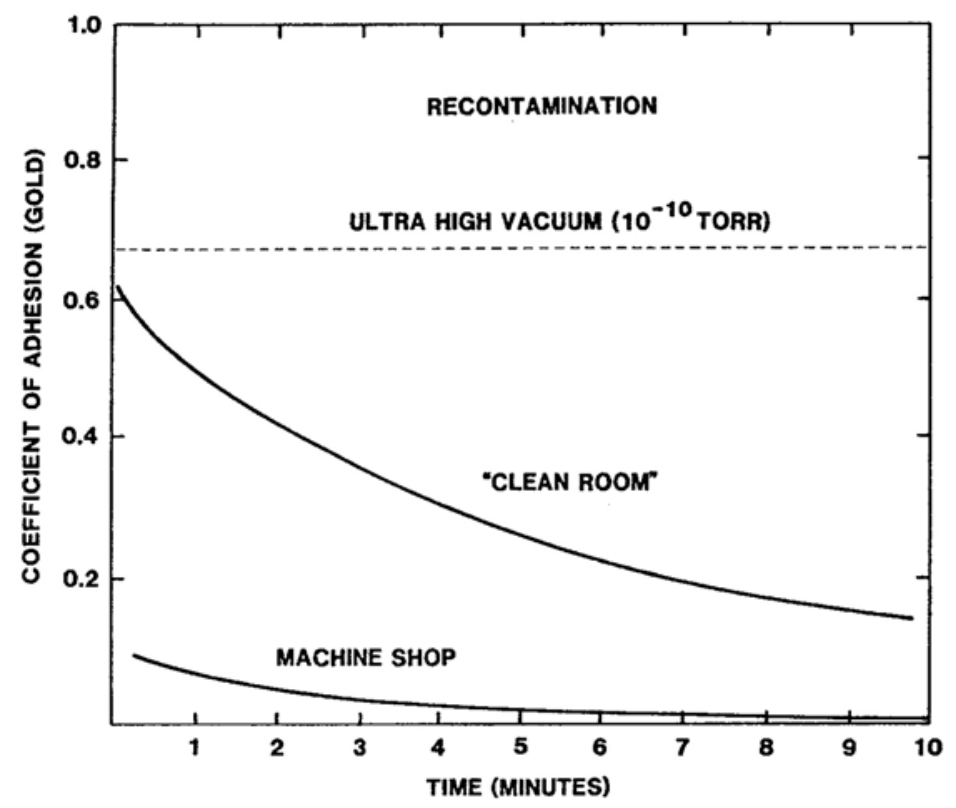

Figure 2.8.1 [8]

\section{- Section 2.9 \\ Characterization}

Characterization is process of determining the physical and chemical properties of a material in a reproducible way. Characterization determines the effect process variables have on the properties of the materials and establishes the performance criteria of the system for satisfactory results. Further characterization of the materials produced is often the first indicator when something has gone wrong with the deposition process.

\section{- Section 2.9.1}

\section{Characterization Using Fourier Transform Infrared Spectroscopy}

Infrared Spectroscopy is a power tool to determine the structure and purity of a compound. In IR Spectroscopy electromagnetic waves of wavelengths between $780 \mathrm{~nm}$ to $300,000 \mathrm{~nm}$ are sent to reflect off of or transmit through a sample so that an absorption 
spectrum is collected. Periods of high absorption in an IR spectrum, referred to as peaks, correspond to the energy that is present between two of the atoms in the compound.

Atoms that are adjacent to one another are not fixed to a single position but vibrate around some equilibrium position. These vibrations that occur are a characteristic of the weight of the atoms involved and the strength of the bonding and are referred to as the "vibrational modes" of the material. These vibrations between atoms can be split into two basic categories; stretching, where two or more atoms or groups of atoms contract and release, and bending, where atoms or groups move while holding some central position. The two classes of vibrations are further broken down into sub categories of vibrations based on symmetric or asymmetric and in-plane or out-of-plane vibrations.

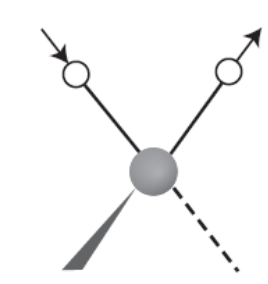

asymmetrical stretching

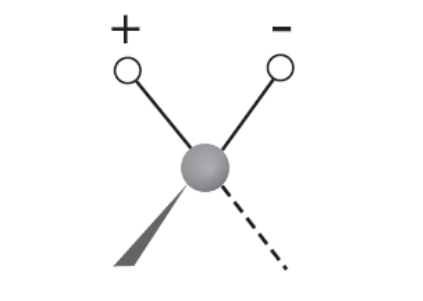

twisting, or bending out-of-plane

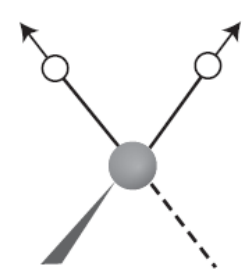

symmetrical stretching

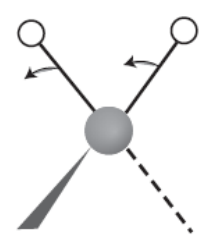

rocking, or bending in-plane

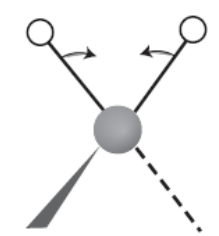

scissoring, or bending in-plane

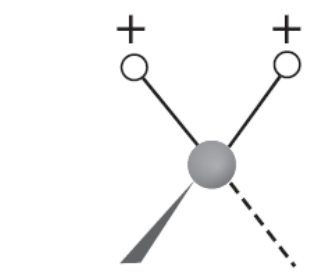

wagging, or bending out-of-plane

Figure 2.9.1 [11]

The number of vibrations that any molecule will have is derived from the number of degrees of freedom that correspond to that molecule. Degrees of freedom are broken into three categories, translational, rotational, and vibrational [14]. Translational movements occur when the atom or molecule as a whole moves in one of the three $\mathrm{x}, \mathrm{y}$, and $\mathrm{z}$ dimensions, rotational movements refer to when the molecule as a whole rotates around some axis, and vibrational degrees of freedom refer to some change in bond 
distance or angle between atoms in the molecule. For a molecule with $n$ atoms there are $3 n$ degrees of freedom, one for each of the three directional axes. For a single atom the number of degrees of freedom found will be three, one for each of the translations directions. For a molecule that contains two atoms the number of degrees of freedom increases to six, again, three of these are lost to translational movements. Assuming that the molecule is symmetric two of the last three are considered rotational modes. The rotation around the axis which goes through the bond does not count since the molecule is completely symmetric surrounding that axis. The last of the degrees of freedom is considered a vibrational mode. If the molecule were not symmetric then three of the degrees of freedom would still be lost to translation modes but now the last three modes would all be lost to the rotational modes since a nonzero moment of inertia would exist for rotation around all axes. In general, a molecule has $3 n-6$ vibrational modes for a nonlinear molecule and $3 n-5$ vibrational modes for linear molecule. In practice this value is often much higher than the number of modes or "peaks" that are seen in an IR spectrum. A bending motion that is in-plane has all the same characteristics as a bending out-of-plane and is labeled as a degenerate mode since they both represent the same energy. Also not all vibrational modes are IR active. For a vibrational mode to be considered IR active a change in the dipole moment, a product of charge and distance of separation, must occur. This is not seen in bonds formed by the same atom such as $\mathrm{N}_{2}$, $\mathrm{H}_{2}$, and $\mathrm{O}_{2}$ as the electron cloud is symmetric and equally shared between both atoms.

Finding the energy associated with bonds is determined by Hooke's Law, the law which relates the energy of a harmonic oscillator where a spring is holding two masses together. The basic form of Hooke's Law is

$$
v=\frac{1}{2 \pi} \sqrt{\frac{k}{m}} \quad \text { Equation 2.9.1 }
$$

$v=$ frequency of vibration

$\mathrm{m}=$ mass of objects

$\mathrm{k}=$ force constant of spring or bond

and $\mathrm{k}$ for the harmonic oscillator is

$$
E=\frac{1}{2} k x^{2}=h v \quad \text { Equation 2.9.2 }
$$


This form of the equation is not particularly useful as the mass of the two atoms will not be equal and no consideration is giving to the quantum nature the displacement of the bond (x) must take. Because the vibrational motion is quantized the energy must take on the form

$$
E=\left(n+\frac{1}{2}\right) h v \quad \text { Equation 2.9.3. }
$$

and the new form of Hooke's Law is found to be

$$
\bar{v}=\frac{1}{2 \pi c} \sqrt{\frac{f\left(m_{1}+m_{2}\right)}{\left(m_{1}+m_{2}\right)}} \quad \text { Equation 2.9.4 }
$$

$\bar{v}=$ frequency of oscillation $\left(\mathrm{cm}^{-1}\right)=\frac{v}{c}$

$\mathrm{m}_{1}$ and $\mathrm{m}_{2}=$ mass of atoms one and two

$\mathrm{c}=$ speed of light $(\mathrm{cm} / \mathrm{s})$

$\mathrm{f}=$ force constant of bond $($ dyne $/ \mathrm{cm})$

The new unit introduced above, $\mathrm{cm}^{-1}$, is referred to as wavenumber and is often used in spectroscopy as it is linear with an increase in bond energy. This model works well for two atoms that are independent from any other atoms but for most molecules this is not the case. When one atom has several vibrational modes with different atoms the modes become coupled and peak locations become shifted. It is this deviation from the normal that provides the greatest insight into what is occurring at the atomic level for a given material. A peak shifting from its nominal value is used as an indicator of numerous things such as bond strain or contamination.

The FT-IR, Fourier Transform Infrared spectrometer is the latest incarnation of the infrared spectrometer. In the past there have been numerous methods to collect an IR spectrum but all have revolved around the concept of using quasimonochromatic light shown through a sample and a detector placed on the opposite side of the sample. The FT-IR provides two main advantages over these traditional spectrometers: first FT-IR systems collect data at all wavelengths at once, the need for prisms or gratings to split a broadband light source is removed and two; due to the way data is collected FT-IR systems can make use of filters to increase the signal-to-noise ratio. 
FT-IR systems can be spilt into four general sections, source, interferometer, samples, and detector.

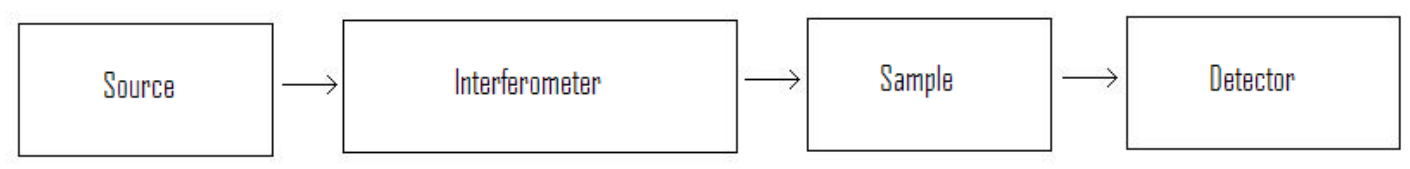

Figure 2.9.2

FT-IR sources are made of some type of heated ceramic. Either a conductive ceramic is used and current is passed through it or a heated filament wrapped around a piece of ceramic is used. As with all hot sources emission of photons increases with temperature and for stable results the source is usually seated inside a protective covering to prevent wind drafts from affecting the source's temperature. A typical FT-IR source is kept around $1000^{\circ} \mathrm{C}$.

The interferometer is the central part of the FT-IR. This is the component that allows the system to essentially look at all wavelengths of light at once.

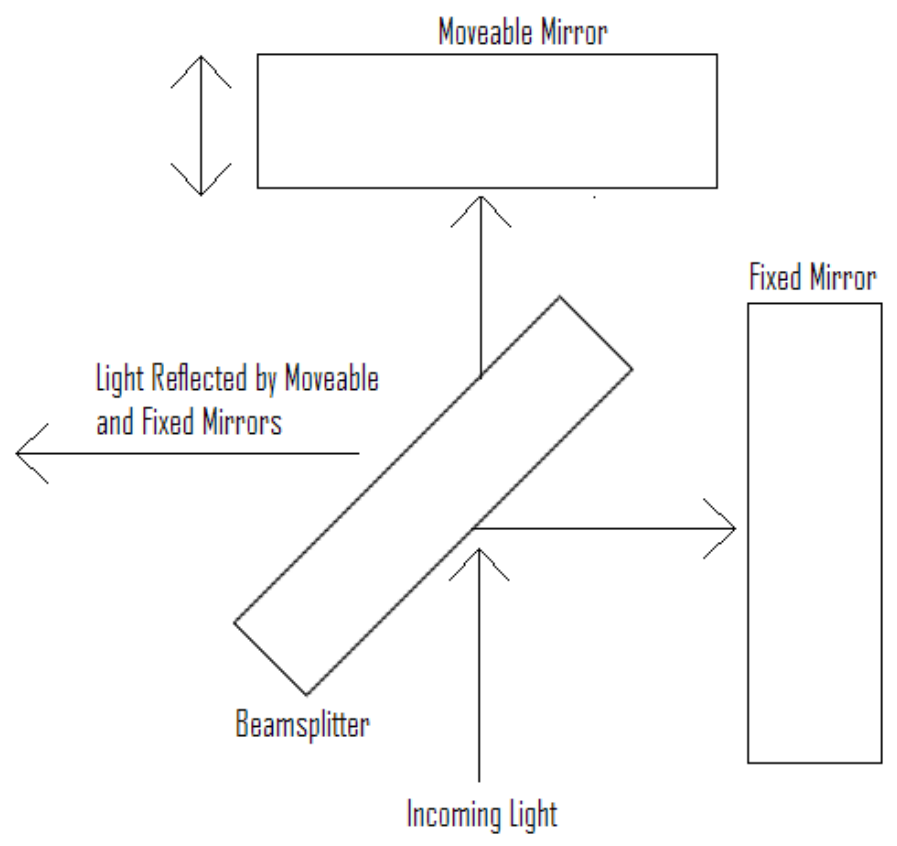

Figure 2.9.3 
The basic Michelson Interferometer has three components, a fixed mirror, a moving mirror, and a beamsplitter. Beamsplitters are used to split incoming light into two rays, one transmitted through and one reflected off of the beamsplitter. The two paths of light will then reach one of two mirrors, be reflected, and travel back towards the beamsplitter. The light will again either be reflected off of or transmitted through the beamsplitter. The ray of light that is of interest for the interferometer is the light ray that is traveling away from the incoming light and the two mirrors, this beam of light consists of what was two separate beams; one which was reflected $90^{\circ}$ from the beamsplitter, reflected $180^{\circ}$ from the fixed mirror and then transmitted through the beamsplitter and a second beam which was transmitted through the beamsplitter reflected $180^{\circ}$ off of the moving mirror and then reflect $90^{\circ}$ off of the beamsplitter. Assuming that the distance traveled by the ray is equal then there should be no difference in the beams when they recombine beyond the beamsplitter. All the different wavelengths of light are completely in-phase and constructive interference occurs. This location of zero path difference between the two light rays is known as the centerburst of an interferogram. An interferogram is a graph of intensity of resulting light versus displacement of the moving mirror. As the mirror moves off of the position where the path difference between the two rays of light is zero destructive interference between the light rays will occur. Once the mirror has traveled so far that the second ray of light has a phase shift of $2 \pi$ the light will again constructively interfere.

$633 \mathrm{~nm}$ Wavelength Light in Interferometer

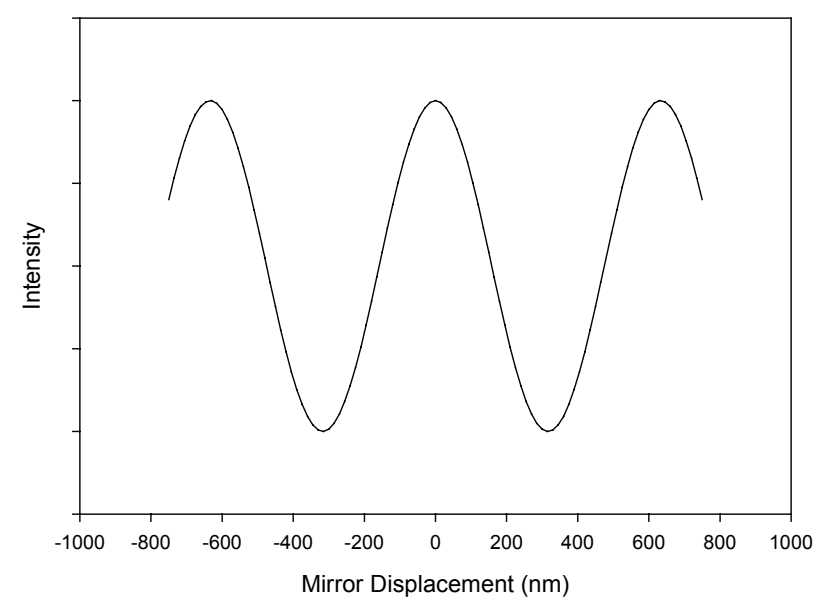

Figure 2.9.4 
If light of more than one wavelength is traveling through the interferometer then a phase shift of $2 \pi$ will not occur at the same mirror displacement for each wavelength and a graph of intensity versus mirror displacement starts to take the shape of a typical interferogram.

$633 \mathrm{~nm}, 850 \mathrm{~nm}, 1100 \mathrm{~nm}$, and $1700 \mathrm{~nm}$ Wavelength Light in Interferometer

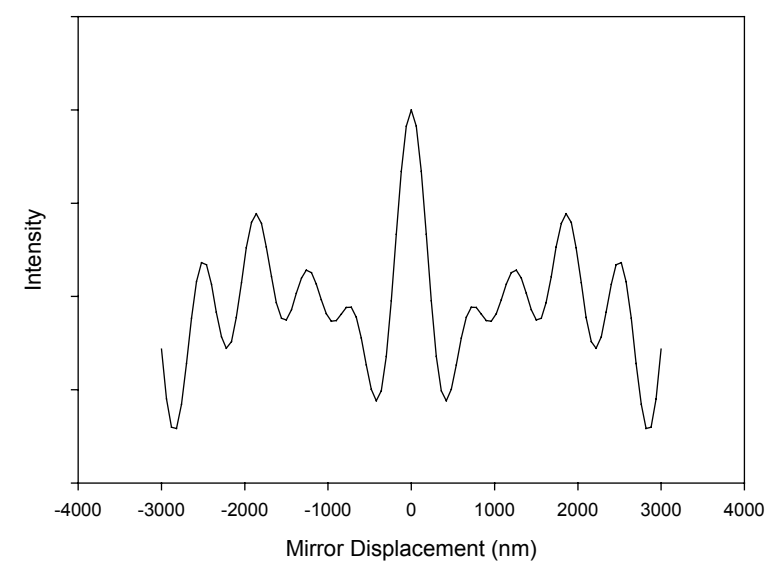

Figure 2.9.5

When all wavelengths of light generated by the source are present the interferogram takes the shape

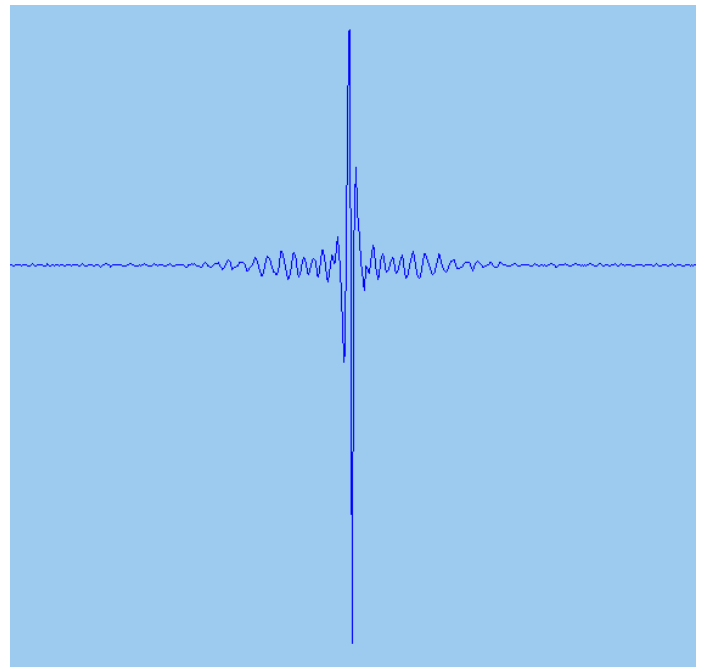

Figure 2.9.6 
Collecting the interferrogram and relating that to mirror displacement is critical to the operation of an FT-IR system. Small changes in mirror displacement must be detected, the system must accurately move a mirror a distance of only a few hundred nanometers. To accomplish such accuracies the FT-IR will send a beam on monochromatic light along with the IR source beam. This light beam, usually a $633 \mathrm{~nm}$ HeNe laser, goes through the same constructive and destructive interference as the IR light traveling through the interferometer. The mirror is therefore not stepped a certain distance but is allowed to continuously move back and forth. The points where the $\mathrm{HeNe}$ light completely disappears is referred to as a zero crossing and allows the FT-IR system to accurately determine the position of the moving mirror. It is at this point that an analog-to-digital converter inside the system reads the signal level on its detector and stores it. This interferogram will now be discrete-time Fourier Transformed which converts this mirror-distance versus signal graph to a frequency versus signal graph.

\section{- Section 2.9.2}

\section{Characterization using Ellipsometry}

Ellipsometry is a powerful tool using polarized light to study thin films and a materials surface [15]. By studying the polarization of light that is reflected off a surface, information can be gained about the properties of the reflective surface if the original polarization of the light was known. A plane electromagnetic wave traveling in the zdirection can be described by the equation [13]

$$
E(z, t)=E_{o} \sin \left(-\frac{2 \pi}{\lambda}(z-v t)+\zeta\right) \quad \text { Equation 2.9.5 }
$$

where

$\lambda=$ wavelength of the wave

$v=$ velocity of wave

$E_{o}=$ wave amplitude

$\xi=$ phase

Looking down the z-axis at the $\mathrm{x}-\mathrm{y}$ plane the electromagnetic wave appears as shown in figure 2.9.7, 


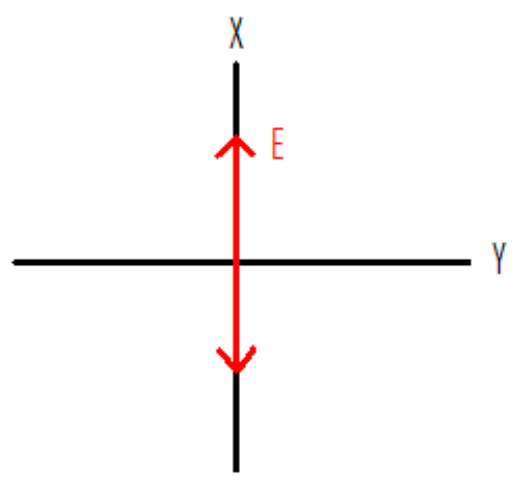

Figure 2.9.7

which is called linearly polarized light. If the angle of the linearly polarized electromagnetic wave does not fall on one of the axes we have arbitrarily chosen as our coordinate system then the wave can be broken into $\mathrm{E}_{\mathrm{x}}$ and $\mathrm{E}_{\mathrm{y}}$ components.

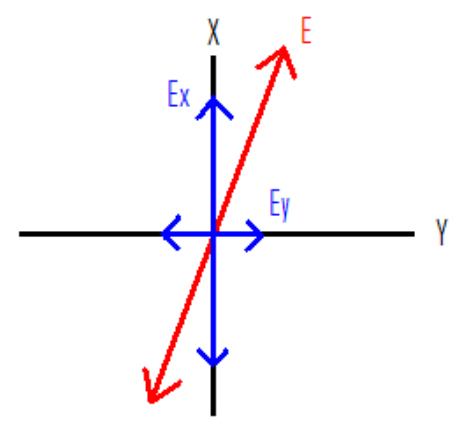

Figure 2.9.8

Although broken into two different waves, these waves represent one linearly polarized traveling wave and are in-phase and traveling in the same direction.

When two or more waves are traveling in the same direction but do not share common amplitude or phase elliptically polarized light occurs. 


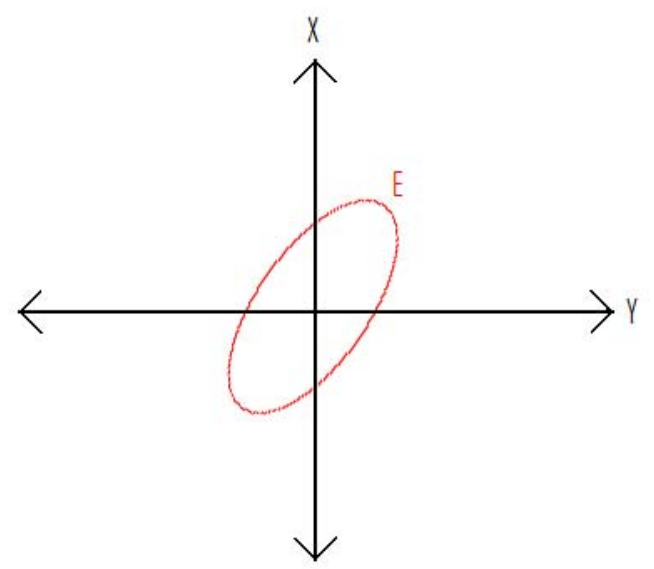

Figure 2.9.9

When linearly polarized light reflects off an interface a change in the polarization direction may occur. For samples with multiple interfaces such as a thin film on a substrate, light will be reflected off of those interfaces with different phases and amplitudes and will sum to form an elliptically polarized light wave. Studying the shape of this ellipse is the theory of ellipsometry.

The two linear components of the incident light use in ellipsometry are referred to as spolarized (perpendicular to plane of incident) and p-polarized (parallel to the plane of incidence). At angles other than $0^{\circ}$ and $90^{\circ}$ the reflection of s-polarized light and ppolarized light will not be the same. The reflection coefficients for a bulk substrate are

$$
R^{p}=\frac{n_{s} \cos \theta_{a}-n_{a} \cos \theta_{s}}{n_{s} \cos \theta_{a}+n_{a} \cos \theta_{s}} \quad \text { Equation 2.9.6 }
$$

and

$$
R^{s}=\frac{n_{a} \cos \theta_{a}-n_{s} \cos \theta_{s}}{n_{a} \cos \theta_{a}+n_{s} \cos \theta_{s}} \quad \text { Equation 2.9.7 }
$$




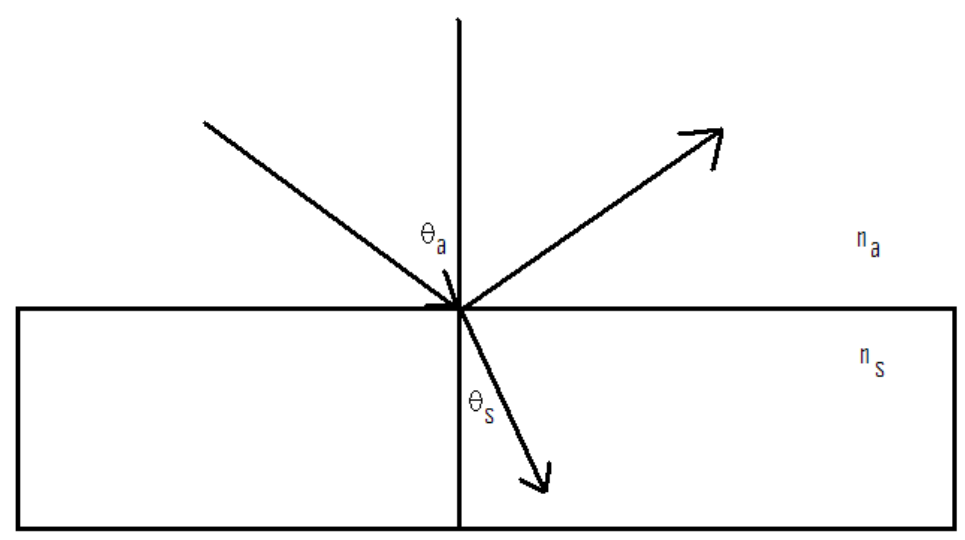

Equation 2.9.10

This ratio is found in the ellipsometer through the equation

$$
\rho=\frac{R_{p}}{R_{s}}=\tan (\psi) e^{i \Delta} \quad \text { Equation 2.9.8, }
$$

$\psi$ being the ratio of the amplitudes and $\Delta$ being the phase difference which the ellipsometer finds by using the shape of the elliptical light wave returned to the detector [16].

A reflection will occur for every interface the incident light reaches, when multiple thin films are added on top of a substrate the overall reflections coefficients can be found by matrix multiplication of successive layer and interface matrices.

$$
\begin{array}{cc}
I_{a b}=\left(\frac{1}{t_{a b}}\right)\left[\begin{array}{cc}
1 & r_{a b} \\
r_{a b} & 1
\end{array}\right] & \text { Equation 2.9.10 } \\
L=\left[\begin{array}{cc}
e^{j \beta} & 0 \\
0 & e^{j \beta}
\end{array}\right] \quad \text { Equation 2.9.11 } \\
\beta_{i}=2 \pi\left(\frac{d_{i}}{\lambda}\right) n_{i} \cos \theta_{i} & \text { Equation 2.9.12. }
\end{array}
$$

$\mathrm{I}_{\mathrm{ab}}=$ interface matrix for $\mathrm{a}-\mathrm{b}$ interface

$\mathrm{L}=$ layer matrix

$\beta_{\mathrm{i}}=$ phase shift due to $i^{\text {th }}$ layer 
$\mathrm{d}_{\mathrm{i}}=$ thickness of $\mathrm{i}^{\text {th }}$ layer

$\theta_{\mathrm{i}}=$ angle of incidence for light on $\mathrm{i}^{\text {th }}$ layer

$\mathrm{n}_{\mathrm{i}}=$ index of refraction of $\mathrm{i}^{\text {th }}$ layer

$r_{a b}=$ reflection coefficient of $i^{\text {th }}$ interface

$t_{a b}=$ transmission coefficient of $i^{\text {th }}$ interface

In many instances the index of refraction has a complex component. This component is directly related to the amount of power absorbed by the material per unit time and unit volume, or loss. Although often the real and imaginary components of the index of refraction are considered independently they are not. The Kramers-Kronig method relates the these two values using the equations [17]

$$
\begin{array}{cc}
f_{1}(\omega)=\frac{2}{\pi} \int_{0}^{\infty} \frac{\omega^{\prime} f_{2}\left(\omega^{\prime}\right) d \omega^{\prime}}{\omega^{2}-\omega^{\prime 2}} & \text { Equation 2.9.13 } \\
\text { and } & \\
f_{2}(\omega)=\frac{2}{\pi} \int_{0}^{\infty} \frac{f_{1}\left(\omega^{\prime}\right) d \omega^{\prime}}{\omega^{2}-\omega^{\prime 2}} & \text { Equation 2.9.14 }
\end{array}
$$

where

$$
\mathrm{f}(\omega)=\mathrm{f}_{1}(\omega)+i \mathrm{f}_{2}(\omega)=\varepsilon(\omega) / \varepsilon_{\mathrm{o}}-1 \quad \text { Equation 2.9.15 }
$$

This is important in optical measurements where the material has a large absorption value, as n will show a strong dependence on the Kramers-Kronig relation.

For a structure which has multiple layers the number of variable that need to be solved for becomes too large to accurately determine the value for each variable. Instead of solving for unknowns, modeling software requires the operator to create a model of what the structure most likely resembles, the software does not solve for a value but simply performs a regression analysis on the model the user has created to determine the most accurate values based on the data it has collected.

Because the values determined by the ellipsometer are found from a mathematical model some type of regression analysis must be performed to optimize the model. The regression analysis attempts to bring to zero the mean square error (MSE) between the 
experimental data and the data from the model. The MSE value is calculated from the function

$$
M S E=\frac{1}{2 N-M} \sum_{i=1}^{N}\left[\left(\frac{\psi_{i}^{\bmod }-\psi_{i}^{\exp }}{\sigma_{\psi}^{\exp }}\right)^{2}+\left(\frac{\Delta_{i}^{\bmod }-\Delta_{i}^{\exp }}{\sigma_{\Delta}^{\exp }}\right)^{2}\right]
$$

$\mathrm{N}=$ number of wavelengths

$\mathrm{M}=$ number of unknowns in model

$\sigma=$ standard deviation of experimental data points

Solving the model requires reducing this value to its absolute minimum; several methods area available to accomplish this minimization [12]. Although several more advanced algorithms are known, many of them are derived from the gradient method which views the MSE as surface in M-dimensional space. The gradient of the MSE surface is a vector with $\mathrm{M}$ components. To move along the gradient, and towards the minimum value, all components of this vector are modified by some multiple of the gradient.

The degree to which an operator can trust the values solved for by the ellipsometer modeling software depends on how much the operator knew about the sample beforehand. Ellipsometric modeling software includes several options which place constraints on the models parameters. A common technique in spectroscopic ellipsometry is to force the model to fit the parameters not at every wavelength but for parameters of a functional based model. For dielectric thin films a common functional based model is the Cauchy model which assumes that the real and imaginary portions of the index of refraction decrease with wavelength based on the functions

$$
\begin{gathered}
n=A_{n}+\frac{B_{n}}{\lambda^{2}}+\frac{C_{n}}{\lambda^{4}} \quad \text { Equation 2.9.17 } \\
\quad \text { and } \\
k=A_{k} e^{-B_{k} \lambda} \quad \text { Equation 2.9.18. }
\end{gathered}
$$

Instead of solving for $\mathrm{n}$ and $\mathrm{k}$ values at every wavelength the modeling software will solve only for $A_{n}, B_{n}, C_{n}, A_{k}$, and $B_{k}$. 
More advanced modeling options are available in modeling software which can be used to study surface morphology and interdiffusion of thin films. The Effective Medium Approximation (EMA) model is used in many instances when a material is composed of two or three different materials. The simplest form is the linear approximation method

$$
\widetilde{\varepsilon}=f_{A} \widetilde{\varepsilon}_{A}+f_{B} \widetilde{\varepsilon}_{B}+f_{C} \widetilde{\varepsilon}_{C}
$$

where $\widetilde{\varepsilon}_{A}, \widetilde{\varepsilon}_{B}$, and $\widetilde{\varepsilon}_{C}$ are the complex dielectric functions of the materials and $\mathrm{f}_{\mathrm{A}}, \mathrm{f}_{\mathrm{B}}$, and $\mathrm{f}_{\mathrm{C}}$ are the fractions of the mixed materials. $\widetilde{\varepsilon}$ is the resulting dielectric function.

Although not extremely accurate, the algorithm is often used for quick calculations.

More computationally expensive methods such as the Maxwell-Garnett and Bruggemann EMA's are commonly seen in model software packages.

Thin films less than $10 \mathrm{~nm}$ are considered "very thin films" and are more difficult to characterize in ellipsometry [18]. Although still very sensitive to the thin film itself, a correlation between index of refraction and thickness is present at these thickness and it is impossible to determine both by ellipsometry alone without some assumption being made. A graph of the $\psi$ and $\Delta$ values for a film between 10 to $80 \mathrm{~nm}$ and indexes of 1.46 to 2.2 on a silicon wafer it can be seen that these values are very sensitive to the $10 \mathrm{~nm}$ film, especially the phase difference coefficient which has changed almost 30 degrees between the silicon wafer and the silicon wafer with the $10 \mathrm{~nm}$ oxide layer.

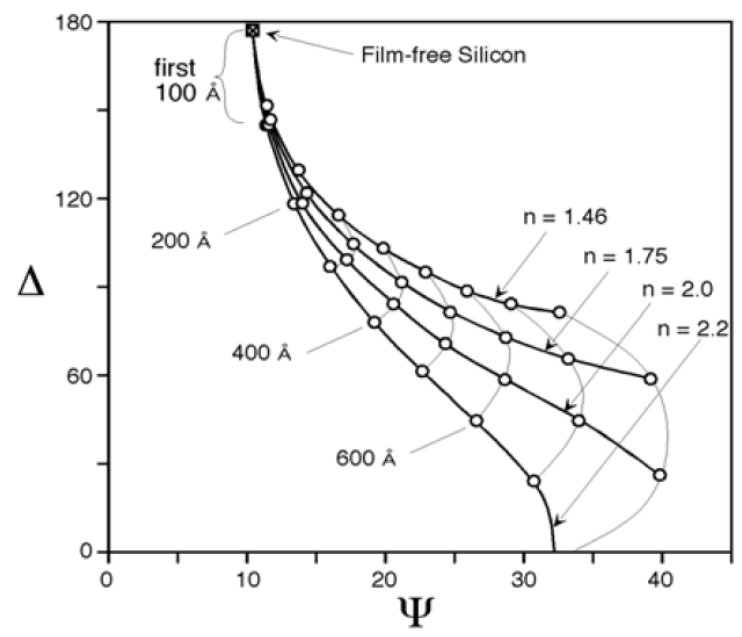

Figure 2.9.11 
For thicknesses above $10 \mathrm{~nm}$ a unique solution is available for all the different thicknesses and indexes. Unfortunately no unique solution is available at the $10 \mathrm{~nm}$ thickness for the various indexes and it is generally not possible to distinguish between correct and incorrect optical constants for very thin films. The most useful solution in these instances is to use the most accurate optical constants available and then fit for an "effective thickness." The "effective thickness" will have some error due to the error in the optical constants but will be reproducible and give good results when relating the thickness of one film to the thickness on another.

\section{Chapter Three - Experimental}

\section{- $\quad$ Section 3.1}

\section{Silicon Dioxide from Electron-Beam Deposition}

The first component of my research was the characterization of silicon dioxide thin films grown from the Temescal BJD-2000 electron gun deposition chamber. Our goal was to grow $\mathrm{SiO}_{2}$ layers with reproducible refractive indices and thicknesses as well as stability to environmental conditions. For simplicity the initial characterization of the layers was performed by depositing the $\mathrm{SiO}_{2}$ onto silicon wafers. Wafers were degreased in acetone for five minutes and then in methanol for five minutes. To remove any native oxide the wafers would then be etched in a 1:1 $\mathrm{H}_{2} \mathrm{O}$ :HF solution and then rinsed in $\mathrm{H}_{2} \mathrm{O}$ for 30 seconds. The wafers would then be loaded directly into the deposition chamber and pump down would begin.

Experiments were conducted after a pump down of more than eight hours. The wafer holder was rotated at $20 \mathrm{rpm}$ and depositions occurred at $10.00 \mathrm{kV} \pm 0.04 \mathrm{kV}$ accelerating voltage using $99.999 \%$ purity $\mathrm{SiO}_{2}$ source material which was refilled after each deposition. Over fifty samples where performed in this way over the course of 
several months in an attempt to understand the growth conditions that most affected the deposited $\mathrm{SiO}_{2}$ films. The standard operating procedure was a pressure of 3.3x10-7 torr, $70 \mathrm{~mA}$ emission current to a thickness deposition of $1 \mu \mathrm{m}$. Most samples where grown using this method in an attempt to determine the repeatability of the system but in several depositions we deviated from these parameters to see what effect this would have on the films.

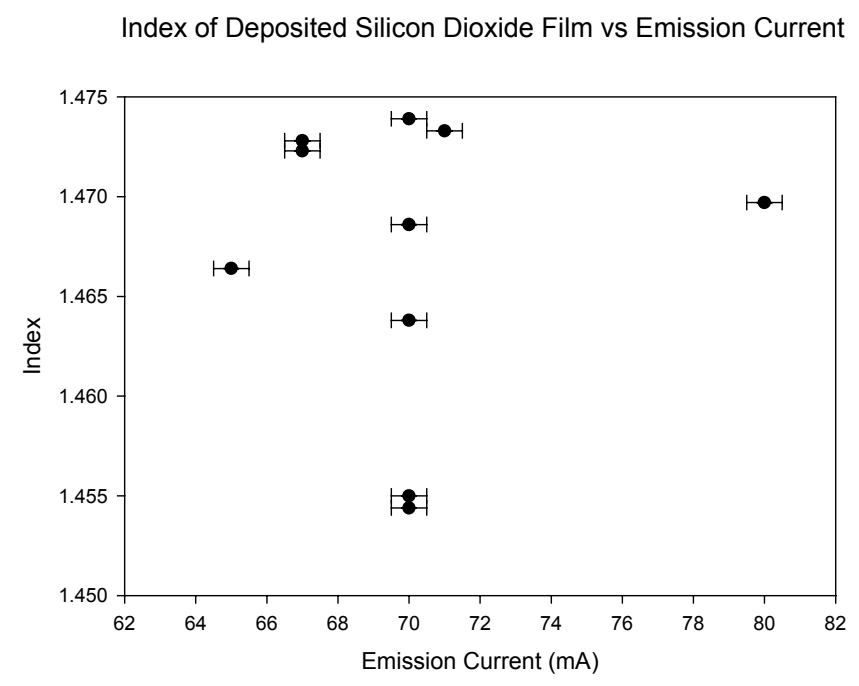

Figure 3.1.1

Chamber Pressure vs. Index of Deposited Film

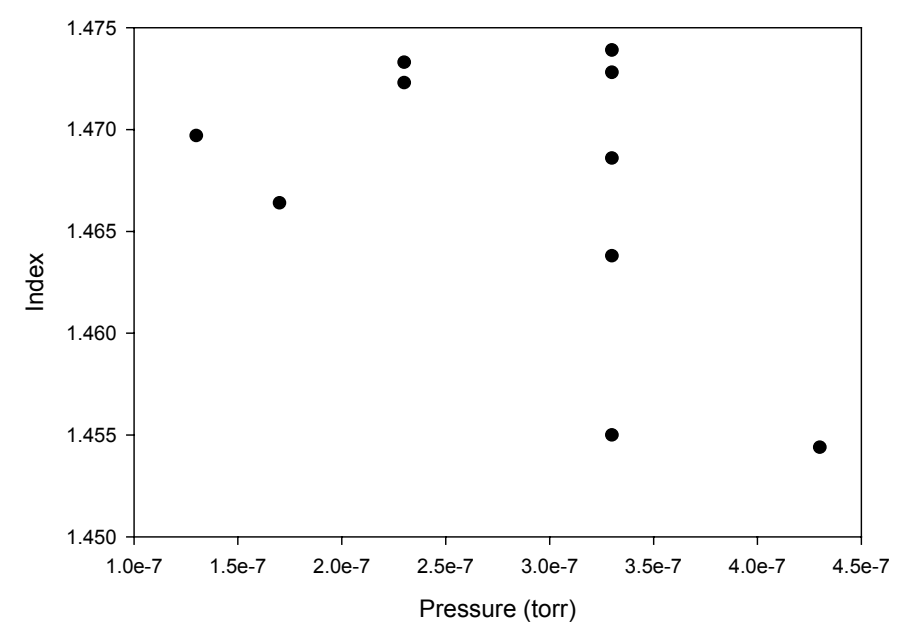

Figure 3.1.2 
Thickness of Silicon Dioxide Films vs Index of Films

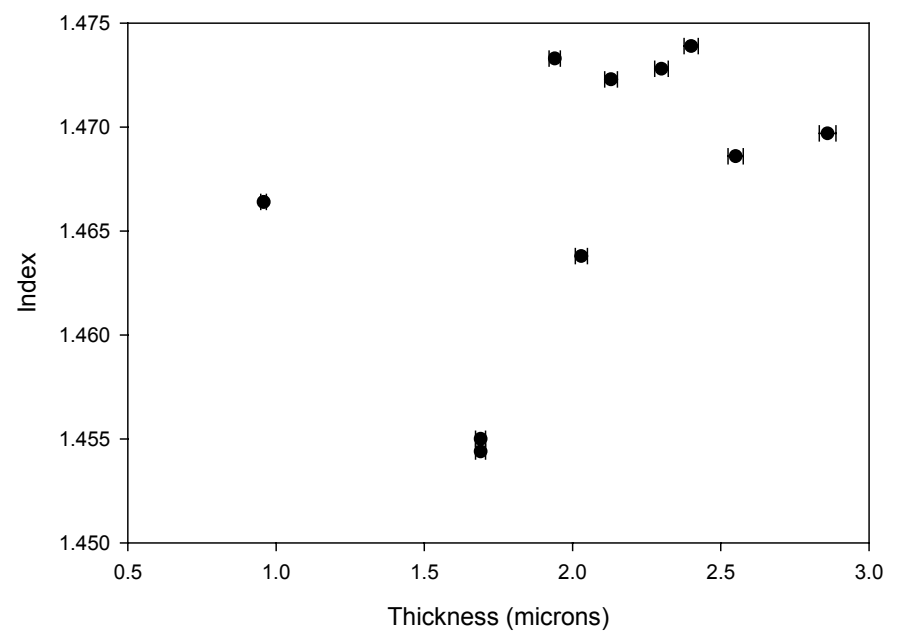

Figure 3.1.3

\begin{tabular}{|c|c|}
\hline$\underline{\text { Sample }}$ & $\underline{\text { Index @ } 633 \mathrm{~nm}}$ \\
\hline 032 & 1.4754 \\
\hline 033 & 1.4780 \\
\hline 047 & 1.4739 \\
\hline 050 & 1.4742 \\
\hline 074 & 1.4544 \\
\hline 075 & 1.4486 \\
\hline Std. Dev & 0.0125 \\
\hline
\end{tabular}

Table 3.1.1

No obvious trend came out of any of these plots. In addition to these parameters we also looked at less obvious criteria such as runs since cyropump regeneration and relative humidity the day the samples was loaded, and the amount of material on the chamber walls but attempts to relate these parameters to the index of refraction where never successful.

The infrared spectrum of silicon dioxide provided a great deal of information about the stoichiometry and stress in the films [19]. Specifically the Si-O in phase stretch 
peak located around $1080 \mathrm{~cm}^{-1}$. A good reference for $\mathrm{SiO}_{2}$ material was the spectrum collected from a thermally oxide wafer.

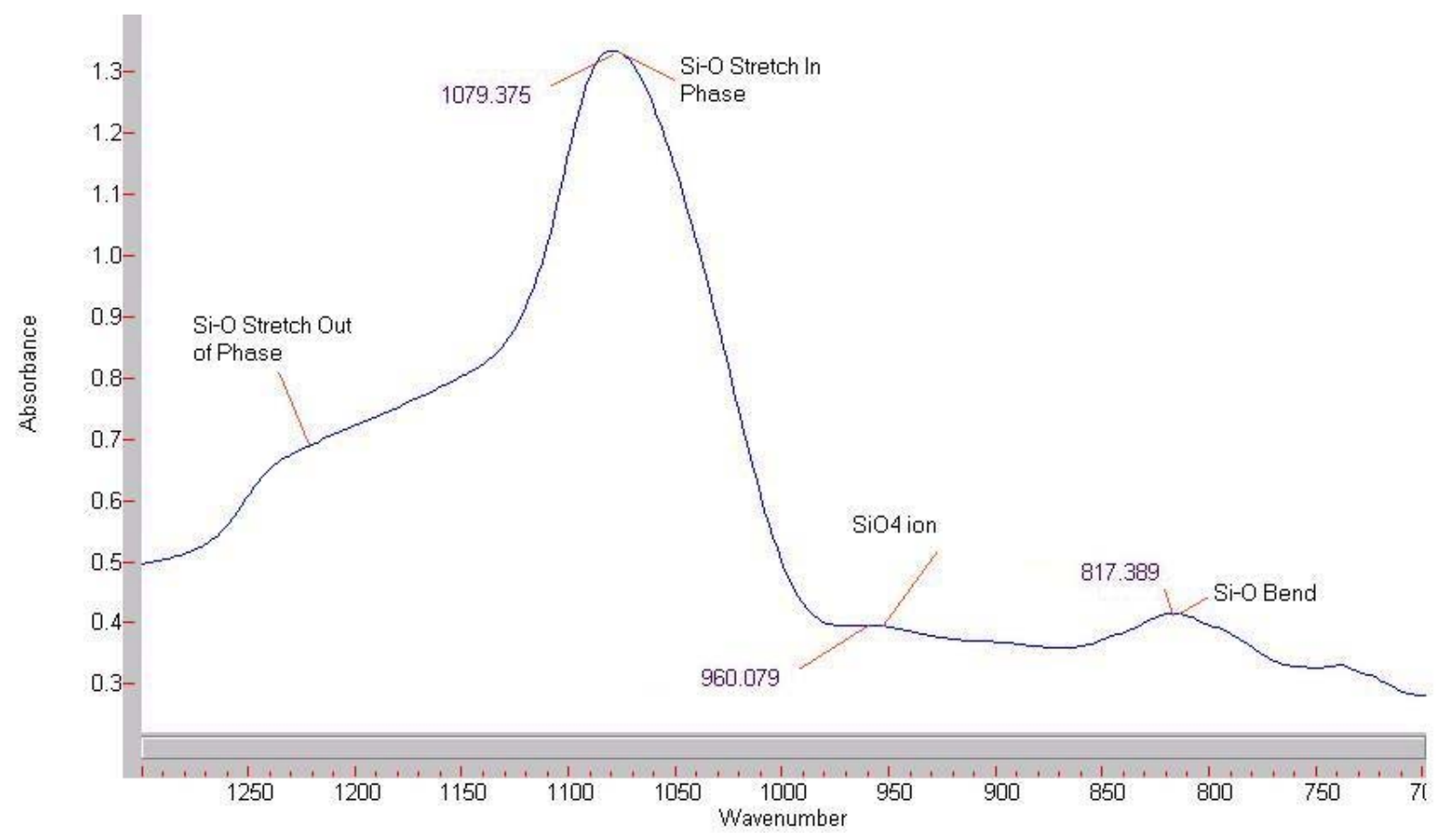

Figure 3.1.4

A thermally grown oxide that is fully relaxed will have a O-Si-O bond angle of $144.7^{\circ}$, a Si-Si distance of $1.60 \AA$, and a Si-O in phase stretch located at $1078.5 \mathrm{~cm}^{-1}$. It is well know that the Si-Si distance and bond angle are related to the strain in the film while these parameters are related to the location of the $\mathrm{Si}-\mathrm{O}$ in phase stretch. The reason for the strain in the films is questionable but is often attributed to a film stoichiometry of less than 1:2 in $\mathrm{SiO}_{2}$.

While performing FTIR studies on the $\mathrm{SiO} 2$ samples it became clear that a shift was occurring in the $\mathrm{Si}-\mathrm{O}$ in phase stretch over time. This effect would be harmful for the biosensor as stability in the environment is a key criteria. 


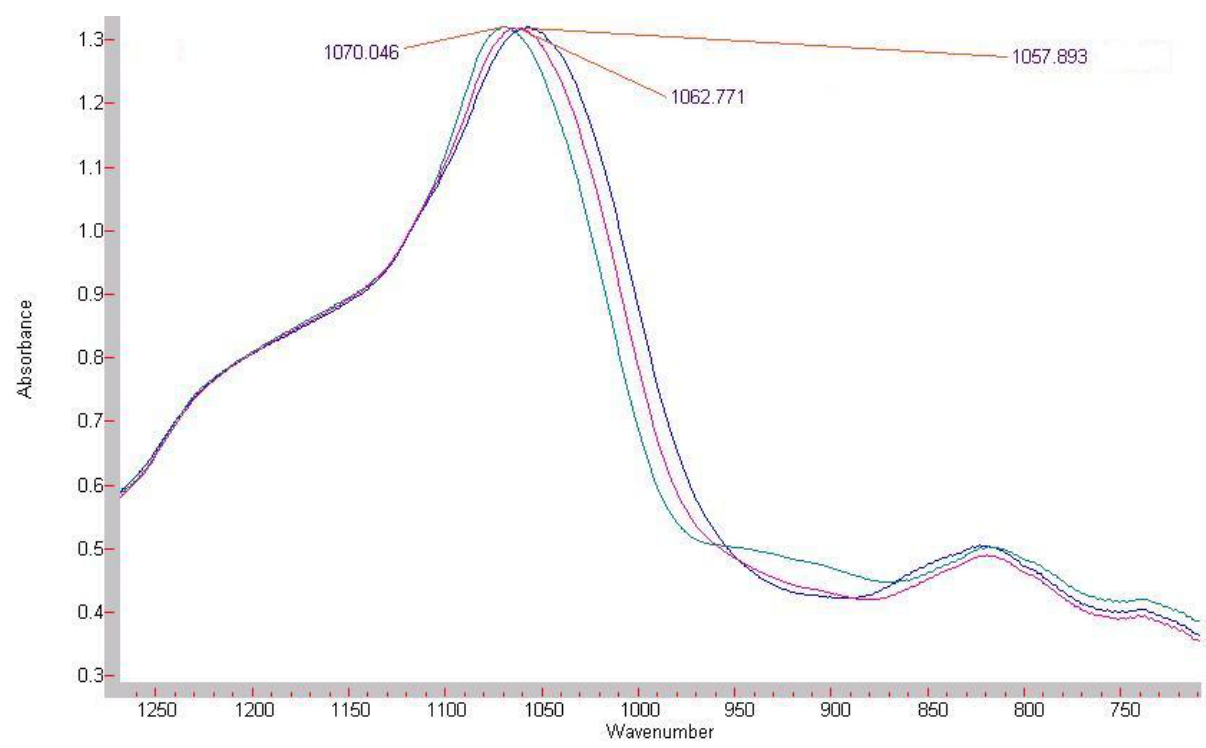

Figure 3.1.5

These three scans of the same sample taken at 0 hours $\left(1057.893 \mathrm{~cm}^{-1}\right), 24$ hours $\left(1062.711 \mathrm{~cm}^{-1}\right)$, and 48 hours $\left(1070.046 \mathrm{~cm}^{-1}\right)$ after they were removed from the deposition chamber show that strain in the film is decreasing as the Si-O in-phase stretch moves closer towards its nominal value of $1078.5 \mathrm{~cm}^{-1}$. There were several possibilities as to why this was occurring: oxidation of the films from $\mathrm{O}_{2}$ in atmosphere, water absorption or desorption from the film, or and a natural relaxation of the $\mathrm{SiO}_{2}$ molecules over time were all considered. In an attempt to study this, three samples where grown in the e-beam chamber during one deposition at $5 \mathrm{~nm} \pm 0.5 \mathrm{~nm}$ per second, $3.3 \times 10^{-7}$ torr, and substrate temperature of $200{ }^{\circ} \mathrm{C}$. FTIR scans were taken in transmission mode at a resolution of $1 \mathrm{~cm}^{-1}$ with a liquid nitrogen cooled MCT detector and an average of 64 scans. Sample A, a reference sample, was removed and taken directly to the FTIR and ellipsometer for study, sample B was placed into a beaker of deionized water for one hour before being taken to the FTIR and ellipsometer for study, and sample $\mathrm{C}$ was left inside the vacuum chamber for 47 hours before it was removed and taken to the FTIR and ellipsometer for study. 


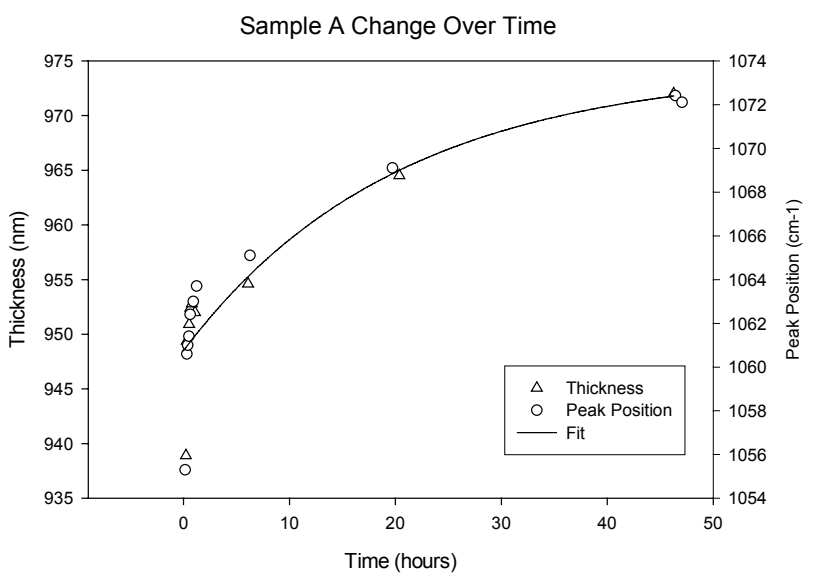

Figure 3.1.6

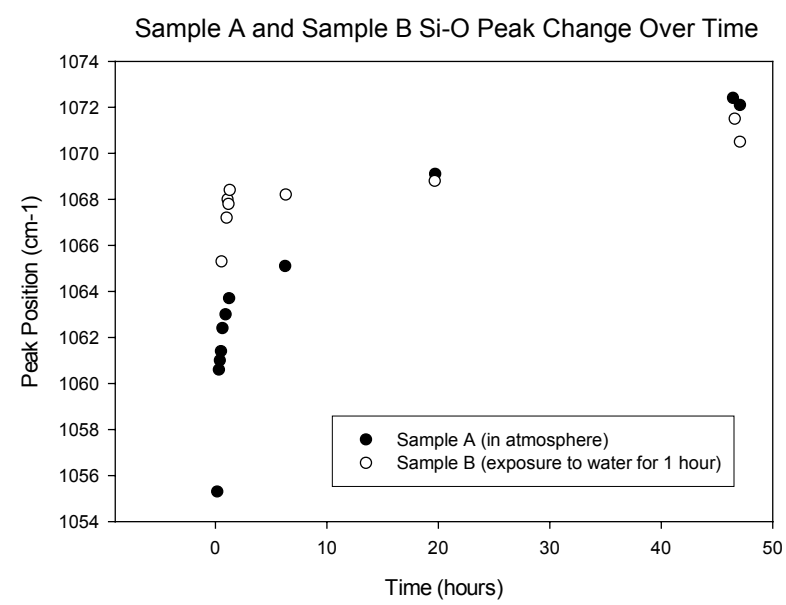

Figure 3.1.7

Sample A and Sample B Thickness Change Over Time

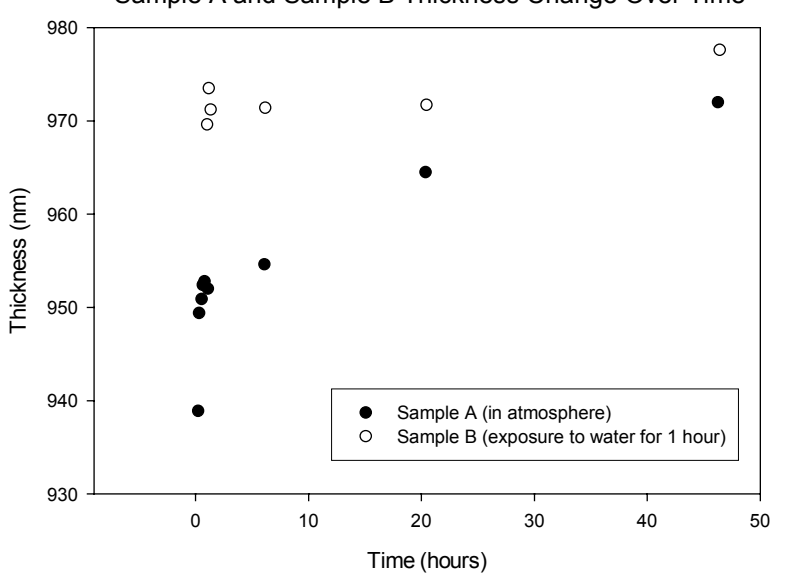

Figure 3.1.8 
The behavior of A showed that a release in strain inside the film was strongly related to an increase in the thickness of the films, in this case about two percent.

Relating B to A it was seen that the deionized water had accelerated the aging effect.

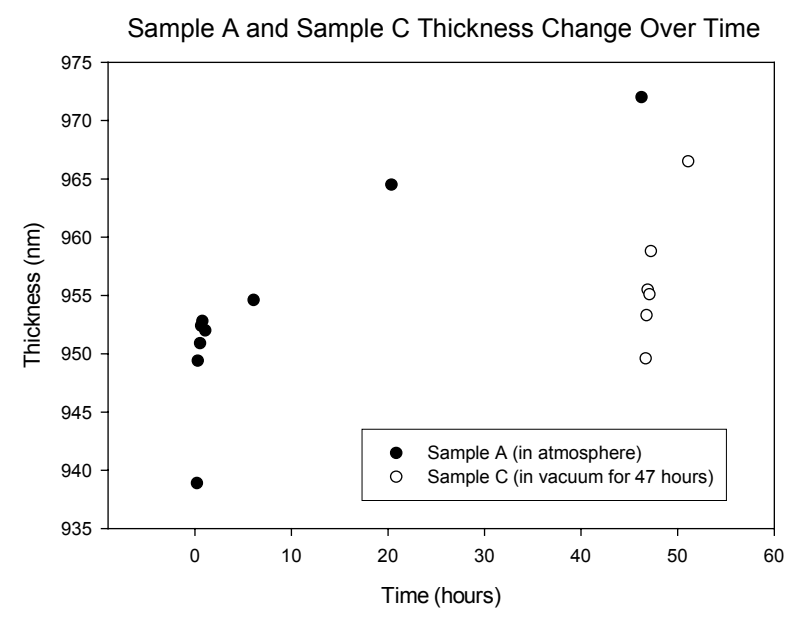

Figure 3.1.9

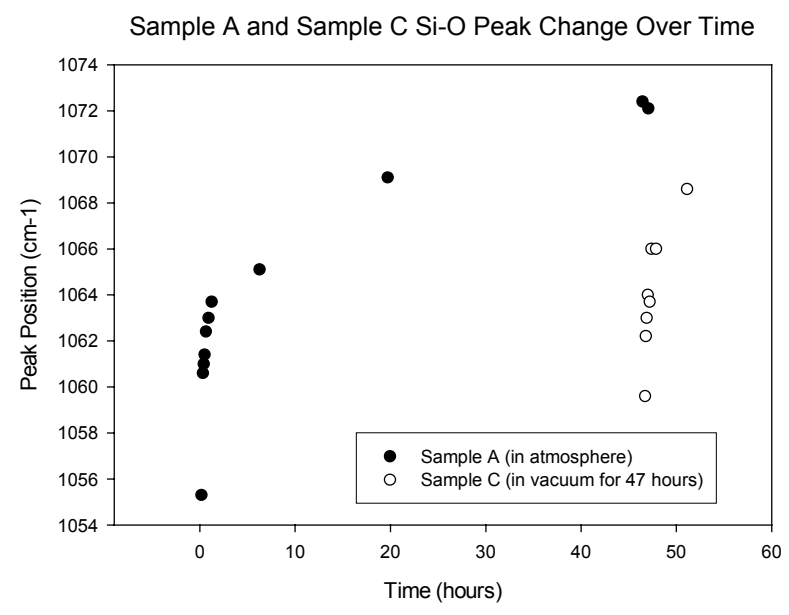

Figure 3.1.10

C showed the exact opposite trend as B; during the 47 hours inside the vacuum chamber almost no change in the thickness or the peak location occurred. The small change that was noticed probably occurred during the time A and B were removed and the chamber was pumping back down. 
Another growth using the same parameters as those for samples A, B, and C was performed. This sample, $\mathrm{D}$, was capped with a $95 \mathrm{~nm}$ layer of $\mathrm{Al}_{2} \mathrm{O}_{3}$ before removal from the chamber. It was scanned immediately after removal and then again scanned seven days later. After this scan the D was placed in deionized water for four hours and then scanned again. These three scans are all within the $1 \mathrm{~cm}^{-1}$ resolution they were collected at $\left(1044.858-1045.514 \mathrm{~cm}^{-1}\right)$ and no shift can be found during this period. The fourth scan was collected 100 days later, its location was found to be $1052 \mathrm{~cm}^{-1}$. This shift was attributed to a release in the stain of the film over time which occurred at a rate much slower than the rate of $\mathrm{SiO}_{2}$ film exposed to atmosphere.

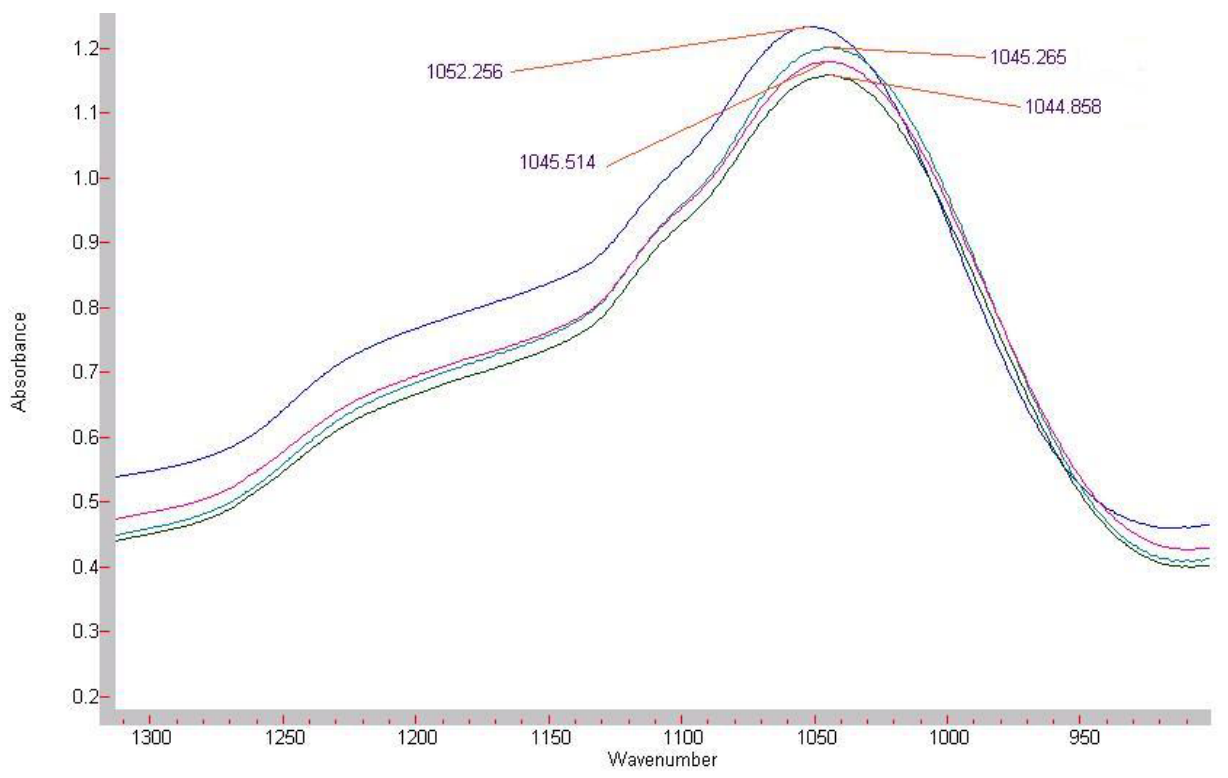

Figure 3.1.11

The final thing that the experiments attempted to prove was that this process is irreversible. A new sample, E, was grown with the same deposition conditions as A through D. E was allowed to age exactly as A had up until day 15. At day $15 \mathrm{E}$ was placed back into the e-beam vacuum chamber and pumped down to $2.7 \times 10^{-7}$ torr for three days. After $\mathrm{E}$ was removed scans were performed in an attempt to witness reversal of the process and a shift of the peak away from its nominal position. Although a small negative shift was noticed between day 15 and day 18 this shift was within the $1 \mathrm{~cm}^{-1}$ resolution of the scan and should not be attributed to anything genuine. 


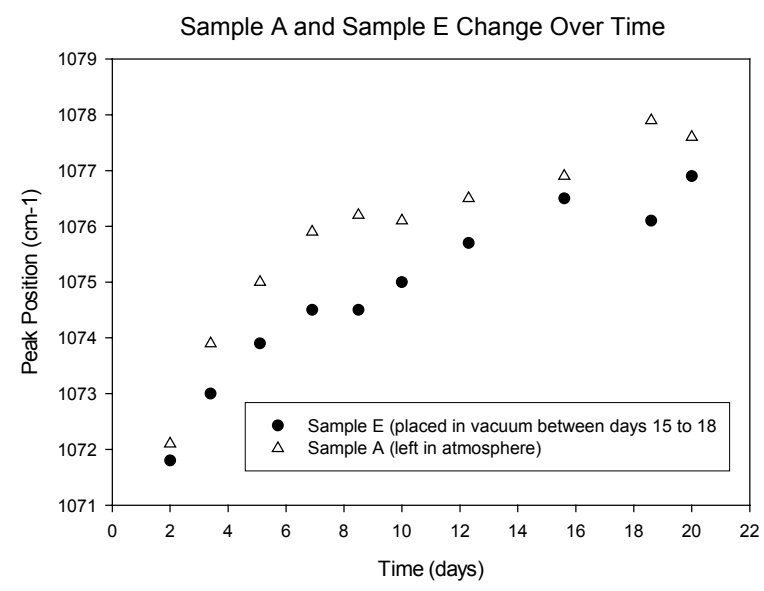

Figure 3.1.12

Determining the reason for these shift and thickness changes was the key to stopping them. Some work has related this process to the water in atmosphere creating $\mathrm{Si}-\mathrm{OH}$ bonds from strained $\mathrm{Si}-\mathrm{O}$ bonds and then reverting back to $\mathrm{Si}-\mathrm{O}$ bonds which are less strained [20]. Others have attributed the strain to water inside the films, desorption of water over time from the film allows it to relax [21]. Clearly in the case of e-beam grown $\mathrm{SiO}_{2}$ films, desorption of water from the film did not cause relaxation, on the contrary the presence of water accelerated the process.

\section{- $\quad$ Section 3.1.1 \\ Silicon Dioxide Depositions with Substrate Heat}

Based on the instability seen in the $\mathrm{SiO}_{2}$ films it was believed that the films grown were very porous. A more dense film would most likely stop or slow the absorption of water into the films. While annealing the films after deposition would increase the density of a porous film, post-deposition annealing can cause cracking when vapor inside the film expands. For higher quality film growth, elevated substrate temperatures are used. This allows surface molecules to diffuse around on the surface and fill any pits that have occurred on the film surface.

The equation 


$$
\Lambda=a \sqrt{\frac{v_{o s} n_{o}}{J_{r}}} e^{-E_{s} / 2 R T} \quad \text { Equation 3.1.1 }
$$

provides a mathematical model explaining why higher substrate temperatures provide better film quality. The diffusion length, $\Lambda$, of the molecule is related to the temperature of the substrate, $\mathrm{T}$, and the deposition flux $\mathrm{J}_{\mathrm{r}}$. The value $v_{\mathrm{os}}$ is the vibrational energy of the molecule in question, although this value only requires attention when the substrate temperature is high enough to move the molecule from its ground vibrational state. For the temperatures used in the e-beam deposition chamber this does not occur and the value is assumed to be $10^{13}$. As the molecule diffuses around the surface it reaches areas of higher and lower surface energy. The value $a$ is the distance between two sites of lower surface energy and $n_{o}$ is the number of these sites per cubic centimeter.

The density of amorphous silicon dioxide is $2.97 \mathrm{~g} / \mathrm{cm}^{3}$. To determine the flux rate for a $10 \AA / s$ deposition we use

$$
\frac{2.97 \frac{\mathrm{g}}{\mathrm{cm}^{3}}}{60 \frac{\mathrm{amu}}{\mathrm{mc}} \cdot 1.66 \times 10^{-24} \frac{\mathrm{g}}{\mathrm{amu}}}=3.0 \times 10^{22} \frac{\mathrm{mc}}{\mathrm{cm}^{3}}
$$

which is the number of molecules per cubic centimeter. The flux is then

$$
3.0 \times 10^{22} \frac{\mathrm{mc}}{\mathrm{cm}^{3}} \cdot 10^{-7} \frac{\mathrm{cm}}{\mathrm{s}}=3 \times 10^{14} \frac{\mathrm{mc}}{\mathrm{cm}^{2} \cdot \mathrm{s}},
$$

assuming no desorption is occurring. If assuming that a site of least surface energy occurs at every $\mathrm{SiO}_{2}$ molecule or between $\mathrm{SiO}_{2}$ every molecule, then the number of surface sites for adsorption is the number of molecules on the surface of the cube used for molecular density so $n_{o}=9.6 \times 10^{14}$ and the distance between them is

$$
a=\frac{1}{\sqrt[3]{3.0 \times 10^{22} \mathrm{~cm}^{3}}}=3.2 \times 10^{-8} \mathrm{~cm} .
$$

$\mathrm{E}_{\mathrm{s}}$ (around $20 \mathrm{~kJ} / \mathrm{mol}$ for amorphous $\mathrm{SiO}_{2}$ ) is the activation energy for surface diffusion on the $\mathrm{SiO}_{2}$ film surface, this value is always much less than the desorption activation energy for $\mathrm{SiO}_{2}$ since bonds are only partially broken as the molecule moves around the surface. $\mathrm{R}$ is the gas constant $0.00831 \mathrm{~J} / \mathrm{mol} \cdot \mathrm{K}$ and varying $\mathrm{T}$ between $273{ }^{\circ} \mathrm{K}$ and 573 ${ }^{\circ} \mathrm{K}$ we find 


\section{Diffusion Length of Silicon Dioxide as a Function of Substrate Temperature}

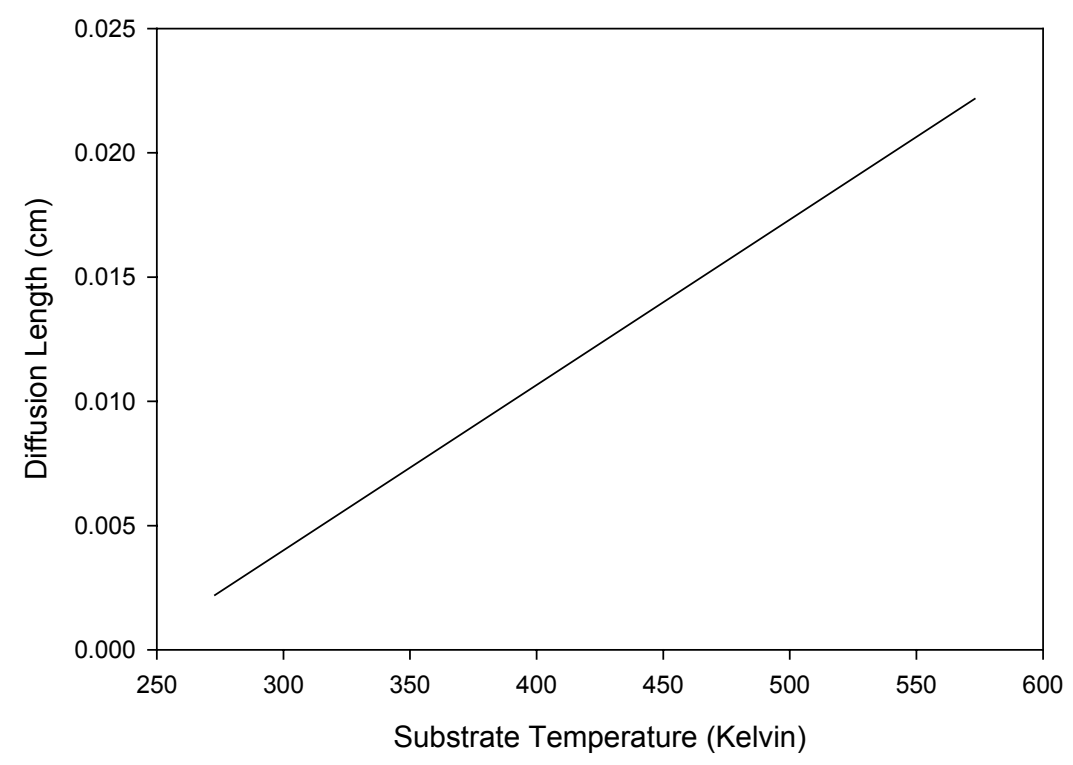

Figure 3.1.13

By increasing the substrate temperature the diffusion length of the $\mathrm{SiO}_{2}$ molecules on the surface has increased from $0.0022 \mathrm{~cm}$ to $0.022 \mathrm{~cm}$. This increased mobility should produce denser films with higher indexes while also reducing the amount of empty space within the films for contaminants to reside. This should in turn produce better overall reproducibility in $\mathrm{SiO}_{2}$ film growth.

The substrate heater gave us one more parameter that could improve film quality. A "bakeout" is simple taking the chamber to a higher temperature than the deposition will be performed at in an attempt to remove contaminants from the surface before deposition. A chamber backout of $290{ }^{\circ} \mathrm{C}$ for ten minutes before lowering the temperature to $280{ }^{\circ} \mathrm{C}$ and starting the deposition allows the chamber pressure to reach pressures lower than it would have been able to achieve if no bakeout would have been performed. 


\begin{tabular}{|l|l|}
\hline$\underline{\text { Sample }}$ & $\underline{\text { Index } @, 633 \mathrm{~nm}}$ \\
\hline S202 & 1.4699 \\
\hline S204 & 1.4669 \\
\hline S206 & 1.4418 \\
\hline S208 & 1.4589 \\
\hline S209 & 1.4690 \\
\hline$\underline{\text { Std. Dev. }}$ & 0.0117 \\
\hline
\end{tabular}

Table 3.1.2

\section{- $\quad$ Section 3.1.2}

\section{Silicon Dioxide Depositions with Ion Beam Assist}

Although the index variations for these samples was still too high for our devices the use of a substrate heater showed promise. A good rule of thumb for improvement in deposited films is to use a substrate temperature of one-third the melting temperature of the material, which for $\mathrm{SiO}_{2}$ is $1713{ }^{\circ} \mathrm{C}$. This means a substrate temperature of $571{ }^{\circ} \mathrm{C}$ would have been a good starting point when attempting to optimize the $\mathrm{SiO}_{2}$. Unfortunately the e-beam deposition chamber was only able to operate at temperatures $<$ $300{ }^{\circ} \mathrm{C}$. A different method to couple energy into the depositions process was needed.

The addition of the Denton Vacuum CC-105 Cold Cathode Ion Source to the ebeam chamber for Ion Beam Assisted Deposition (IBAD) allowed us to add many new deposition parameters, including gas flow into the chamber, drive current of the ions, and neutralizing current. It is well known that the depositions of dielectrics using an e-beam chamber produce porous, non-stoichiometric films. The $\mathrm{CC}-105$ ion source can produce an oxygen ion stream to increase film density and stoichiometry.

The two most important parameters with any ion source flux rate and drive voltage $\left(V_{D}\right)$ which is dependent on the system configuration, drive current, properties of 
the gas in use, gas flow rate, and background pressure. Although the ion source sets $V_{D}$ based on the drive current $\left(I_{D}\right)$ given by the operator, $V_{D}$ can be changed considerably by changing these other parameters. Regardless of the operating parameters the quantities of interest are the energy with which the ions are striking the surface (in $\mathrm{eV}$ ) and the ion flux $\left(\mu \mathrm{A} / \mathrm{cm}^{2}\right)$. The ion source's distance from the center of the chamber, distance from substrate holder, and tilt angle all play important roles in determining these two parameters and ion energy and flux are only determined experimentally.

If ions where allowed to continually strike the substrate a charge would occur and new ions would begin to be repelled. In addition, a positive charge buildup on the chamber walls will cause arcing to occur inside the chamber creating electrical noise in the crystal monitor and possibly tripping the electron guns interlocks. The neutralizing current $\left(\mathrm{I}_{\mathrm{N}}\right)$ and neutralizing voltage $\left(\mathrm{V}_{\mathrm{N}}\right)$ parameter on the ion source controls the amount of current that flows through a filament located just above the head of the ion source and prevents charging from the ions. Electrons enter into the positively charge ion stream and create an electrically neutral plasma. According to literature and the ion sources manual this parameter has no effect of film deposition as long as it is high enough to provide stable ion source and electron gun operation. The user set the $I_{N}$ value and the ion source would adjust the $\mathrm{V}_{\mathrm{N}}$ to continually provide the set value. Because the filament in located directly inside the ion stream its lifetime is very short. When $V_{N}$ reaches $>19 \mathrm{~V}$ filament burnout is imminent.

The use of the ion source required us to rethink the deposition parameters used in the past. The ion source itself produced an appreciable amount of heat which resulted in a steady rise in substrate temperature during deposition. In an attempt to keep the chamber conditions constant throughout the depositions a high substrate temperature was now required. For a typical $1 \mu \mathrm{m} \mathrm{SiO}_{2}$ deposition at different beginning substrate temperatures the final substrate temperatures are listed below. 


\begin{tabular}{|c|c|}
\hline $\begin{array}{c}\text { Beginning Substrate } \\
\underline{\text { Temp }\left({ }^{\circ} \mathrm{C}\right)}\end{array}$ & $\frac{\text { Final Substrate }}{\underline{\text { Temp }\left({ }^{\circ} \mathrm{C}\right)}}$ \\
\hline 100 & 157 \\
\hline 200 & 244 \\
\hline 260 & 271 \\
\hline 280 & 282 \\
\hline
\end{tabular}

Table 3.1.3

For the ion source to be effective the deposition rates were slowed. Because the ion source was off the center axis and the films may not experience the same amount of ion bombardment at different locations in its rotation a deposition of only a few monolayers per revolution was required. Failure to do this would have resulted in alternating layers of different densities inside the film.

Experiments performed to optimize the $\mathrm{SiO}_{2}$ with IBAD growths occurred at a deposition rate of $3.5 \AA / \mathrm{s}$, chamber bakeout at $290{ }^{\circ} \mathrm{C}$ for ten minutes, substrate temperature at deposition of $280{ }^{\circ} \mathrm{C}$, chamber pressure of $8.5 \times 10^{-5}$ (which occurred with a $\mathrm{O}_{2}$ flow rate of $17.5 \mathrm{sccm}$ ), rotation of $50 \mathrm{RPM}$, a neutralizing current of $23.0 \mathrm{~A}$, and a deposition thickness of $1 \mu \mathrm{m}$.

\begin{tabular}{|l|l|}
\hline$\underline{\text { Sample }}$ & $\underline{\text { Index @,633nm }}$ \\
\hline 262 & 1.4637 \\
\hline 263 & 1.4666 \\
\hline Std. Dev. & 0.0021 \\
\hline
\end{tabular}

Table 3.1.4

The sample pool was too small to accurately calculate the standard deviation for $\mathrm{SiO}_{2}$ samples using $\mathrm{SiO}_{2}$ source material and the ion source. The ion source gave us the ability to deposit samples from silicon source material and reactively deposit $\mathrm{SiO}_{2}$. Only 
two samples were grown from $\mathrm{SiO}_{2}$ source material before we switched to the reactive deposition method which is discussed below.

In addition to these characteristics we were also very interested in what would now happen to the Si-O in-phase stretching peak over time. A denser film which has a stoichiometry closer to 1:2 for Si:O should be more stable. This was the case for sample 262 whose FTIR spectrum is below.

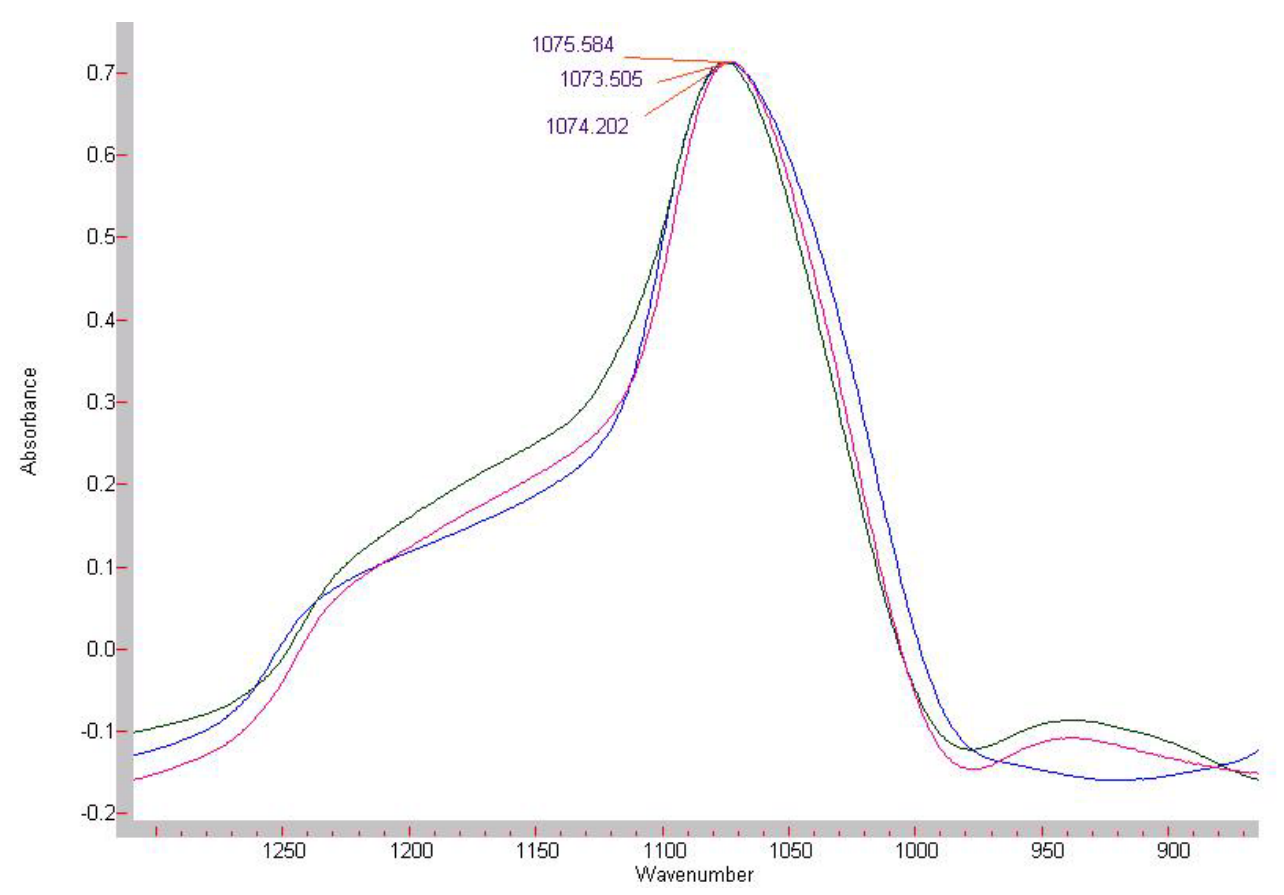

Figure 3.1.14

Although some variation in the FTIR spectrum was noticed and the Si-O stretching peaks were still not at their optimal $1078.5 \mathrm{~cm}^{-1}$ peak the ion source made a great deal of improvement. 


\section{- $\quad$ Section 3.1.3}

\section{Silicon Dioxide Reactive Deposition}

A typical e-beam deposition involves sweeping the beam itself around the source material before deposition begins. The material liquefies and forms a molten pool. Any contaminants in the source material with a higher vapor pressure will be ejected out and stick to the shutter. After a melt in period the beam sweep can be slowed, or even stopped, in the center of the molten pool of source material. Material is evaporated from the center of the pool forming a small pit and new material from the edge flows in to replenish this pit. This technique most accurately represents the model used to characterize and build e-beam chambers, line-of-sight deposition from a point source. Depositing silicon dioxide using an e-beam chamber is considerably different from this ideal case. The thermal characteristics for $\mathrm{SiO}_{2}$ prevent the material from entering into the liquid phase during deposition, the top layer of $\mathrm{SiO} 2$ pellets will fuse together but will not form a molten pool. Deposition flux will fluctuate throughout the deposition as the beam encounters the void between source pellets. In addition the flux change will not be the same for all directions inside the chamber. This introduces error into the correlation between the stationary thickness monitor and the rotating substrates.

A solution to this is to use silicon metal, which does form a molten pool when heated with the e-beam, and perform a reactive deposition with oxygen ions. These depositions where performed at $3.5 \AA / \mathrm{s}$, a chamber bakeout at $290^{\circ} \mathrm{C}$ and deposition at $280^{\circ} \mathrm{C}, 50 \mathrm{RPM}$ substrate rotation, 23.0 A neutralizing current, 2.25 A drive current, 144 $\mathrm{V}$ drive voltage, $17.5 \mathrm{sccm} \mathrm{O}_{2}$ flow which forced a chamber pressure of $8.5 \times 10^{-5}$. This technique produces more accurate thickness than the depositions performed from $\mathrm{SiO}_{2}$ source material but I do not feel that enough depositions performed from $\mathrm{SiO}_{2}$ using similar parameters have been performed to make a mathematical comparison.

Although the depositions from $\mathrm{SiO}_{2}$ with the use of the IBAD produced FTIR spectrum showed very little strain inside the films, it appears that the reactive method produced even less stress inside the films. 


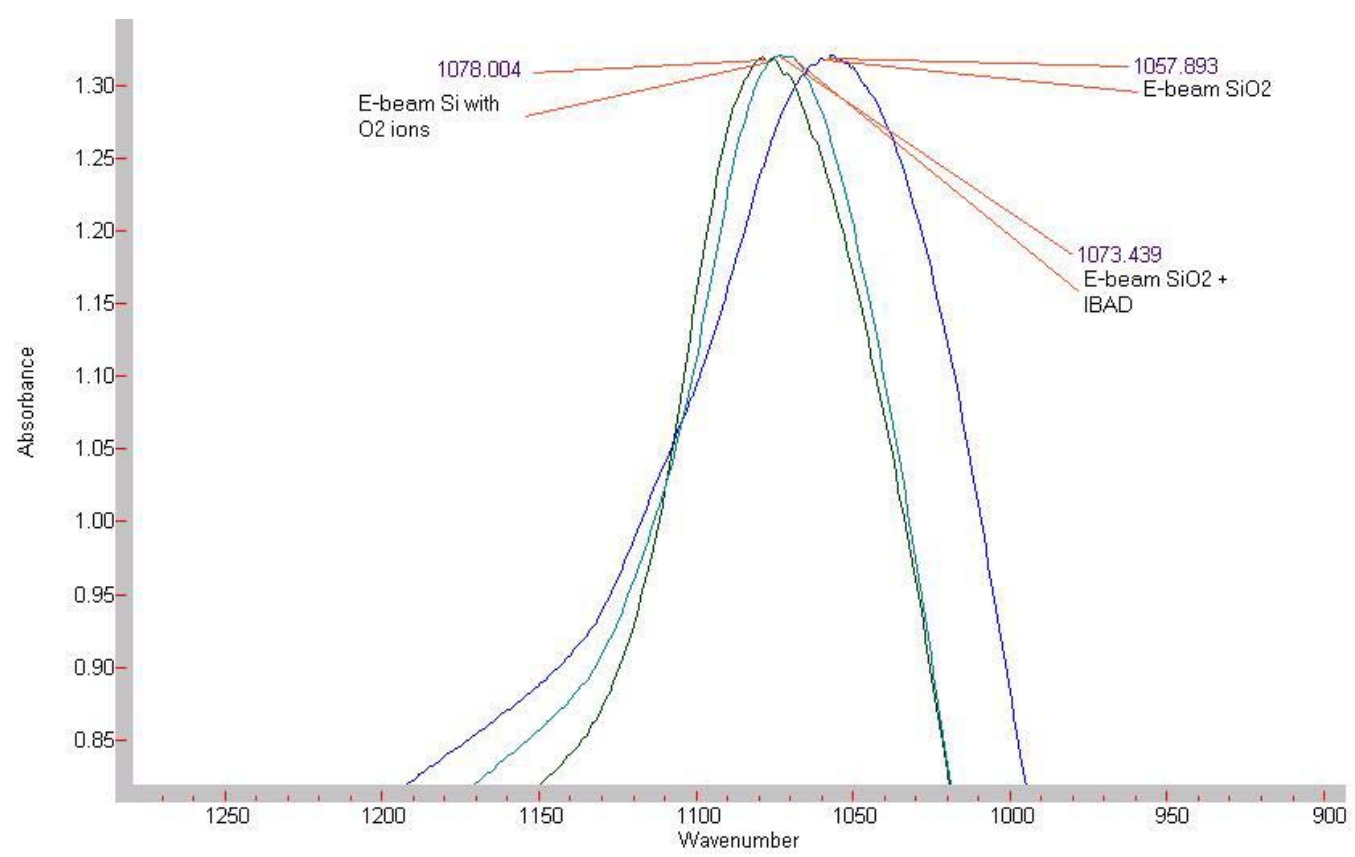

Figure 3.1.15

In addition no peak shift has been detected in the reactively grown $\mathrm{SiO}_{2}$ while samples from $\mathrm{SiO}_{2}$ source with IBAD did move slightly over the course of several months.

\begin{tabular}{|l|l|}
\hline Sample & $\underline{\text { Index }}$ \\
\hline 283 & 1.4768 \\
\hline 284 & 1.4794 \\
\hline 287 & 1.4742 \\
\hline 315 & 1.4762 \\
\hline Std. Dev. & 0.0021 \\
\hline
\end{tabular}

Table 3.1.5

\section{- $\quad$ Section 3.2}

\section{Sweep Pattern Selection}

As discussed before, silicon dioxide material can be difficult to evaporate due to the inability to create a molten pool of source material. It is therefore necessary to create a sweep pattern that will continue throughout the deposition and will evaporate from all 
points on the source material surface equally. If this failed to occur, the electron beam would drill through the source material and begin striking the graphite liner or copper hearth resulting in contamination or damage to the electron gun itself. Although the gun sweeper allows the operator to rapidly create new sweep patterns these are usually only for simple geometries such as circles or figure eight's.

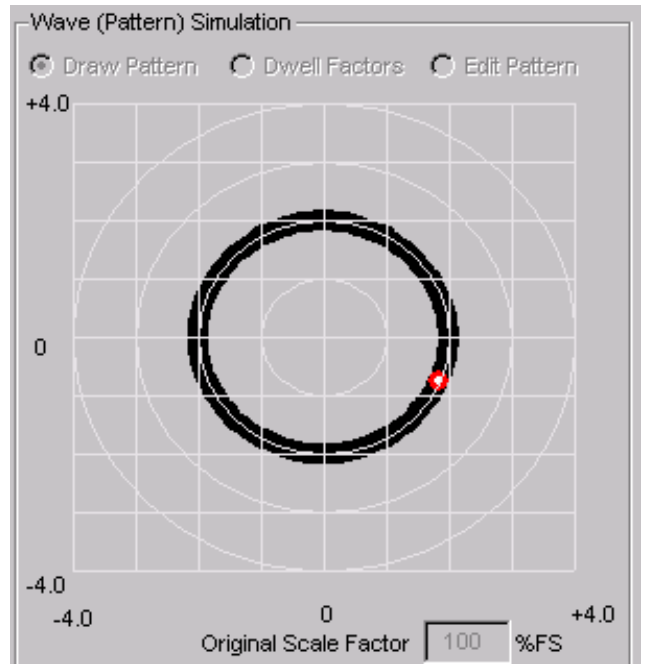

Figure 3.3.1

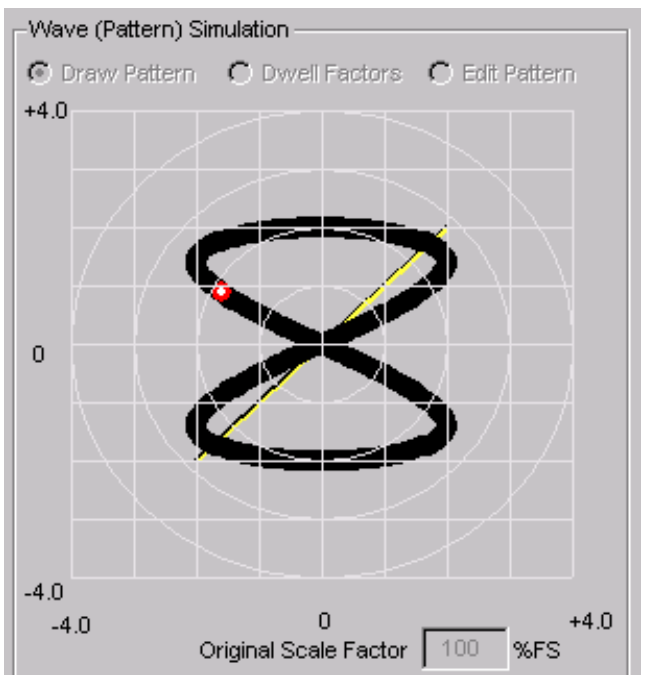

Figure 3.3.2 


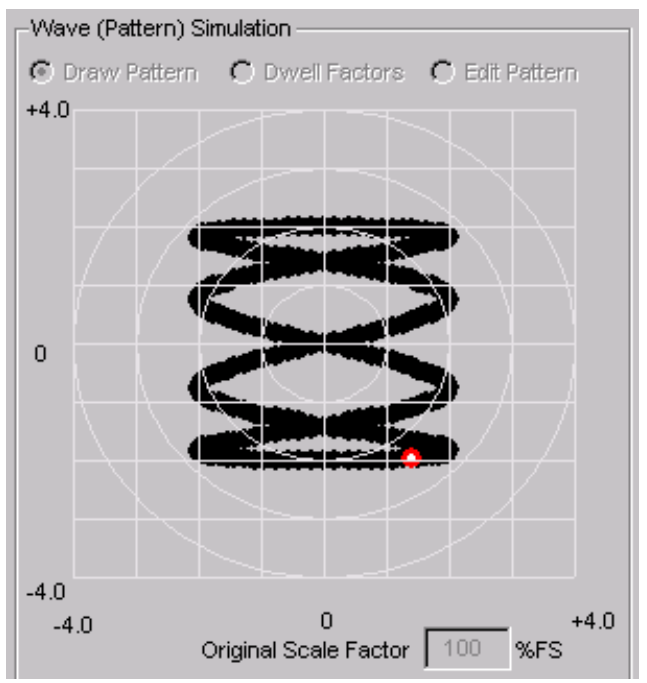

Figure 3.3.3

The patterns all assume that the electron beam itself has a constant shape over the entire pocket which is not the case. The beam itself is focused at the top center of the crucible and becomes more diffused as it travels away from that position. The designer of the ebeam chamber, BOC Edwards/Temescal, provided us with a model for a sweep pattern for subliming materials.

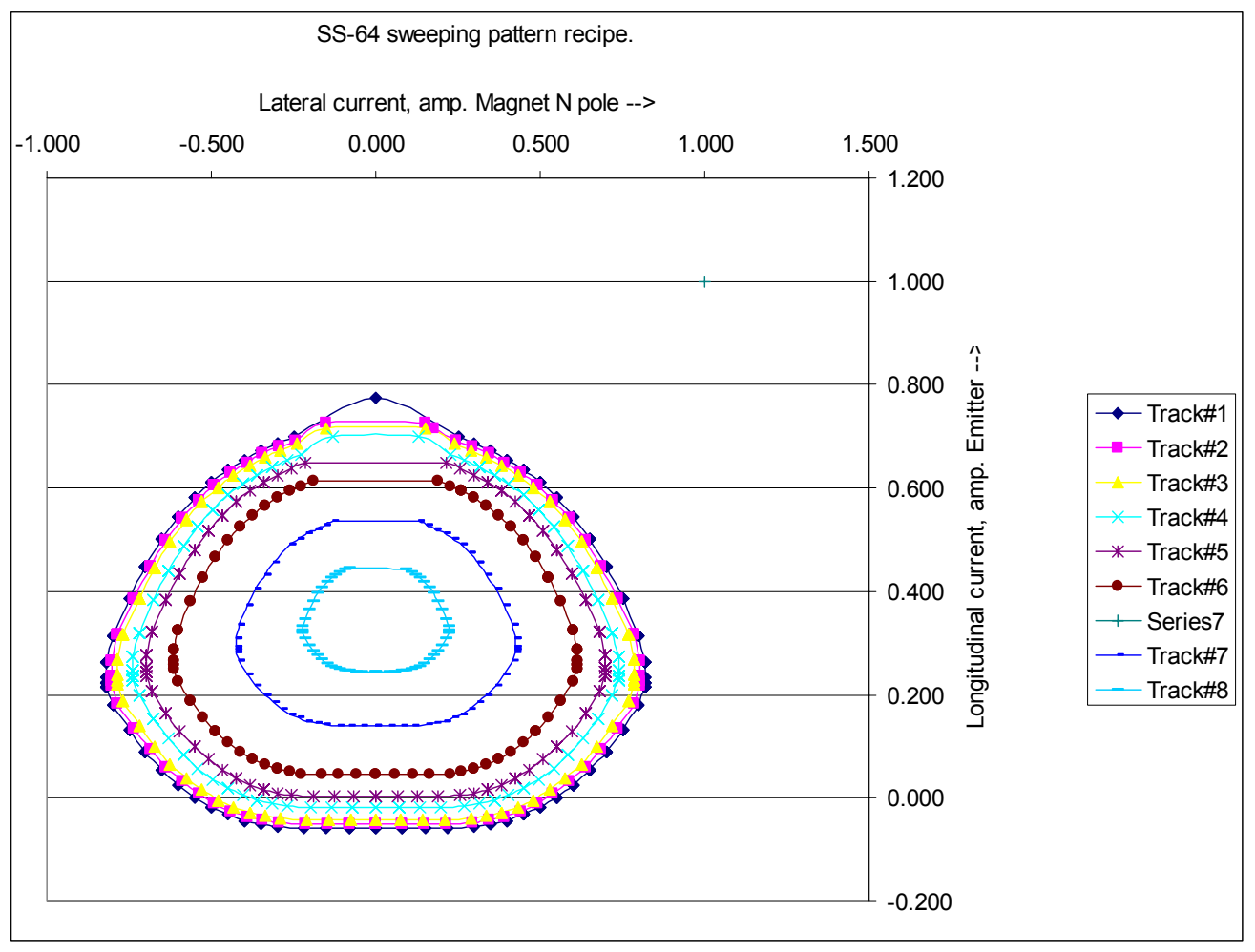

Figure 3.3.4 
For the gun this sweep pattern was created on the default position of the electron beam was center/front of the pocket. The beam moves around to many more points when it is closer to the front to prevent the focused beam from drilling through the source material. As the beam moves towards the back of the pocket it is moved less. At this position the beam is more defused and can reside longer at a single position. Also the beam traverses this track eight times, each time moving closer towards the center while staying less time at each track.

This provides a good model for what was required for the sweep pattern but would not work for our system which had its default position as the center/back. Also since the pocket for the system the model was created on was much larger, eight consecutively smaller tracks were not required for the smaller pockets. A more useful sweep pattern would be one that was inverted along the x-axis.

Inverted Temescal Sweep

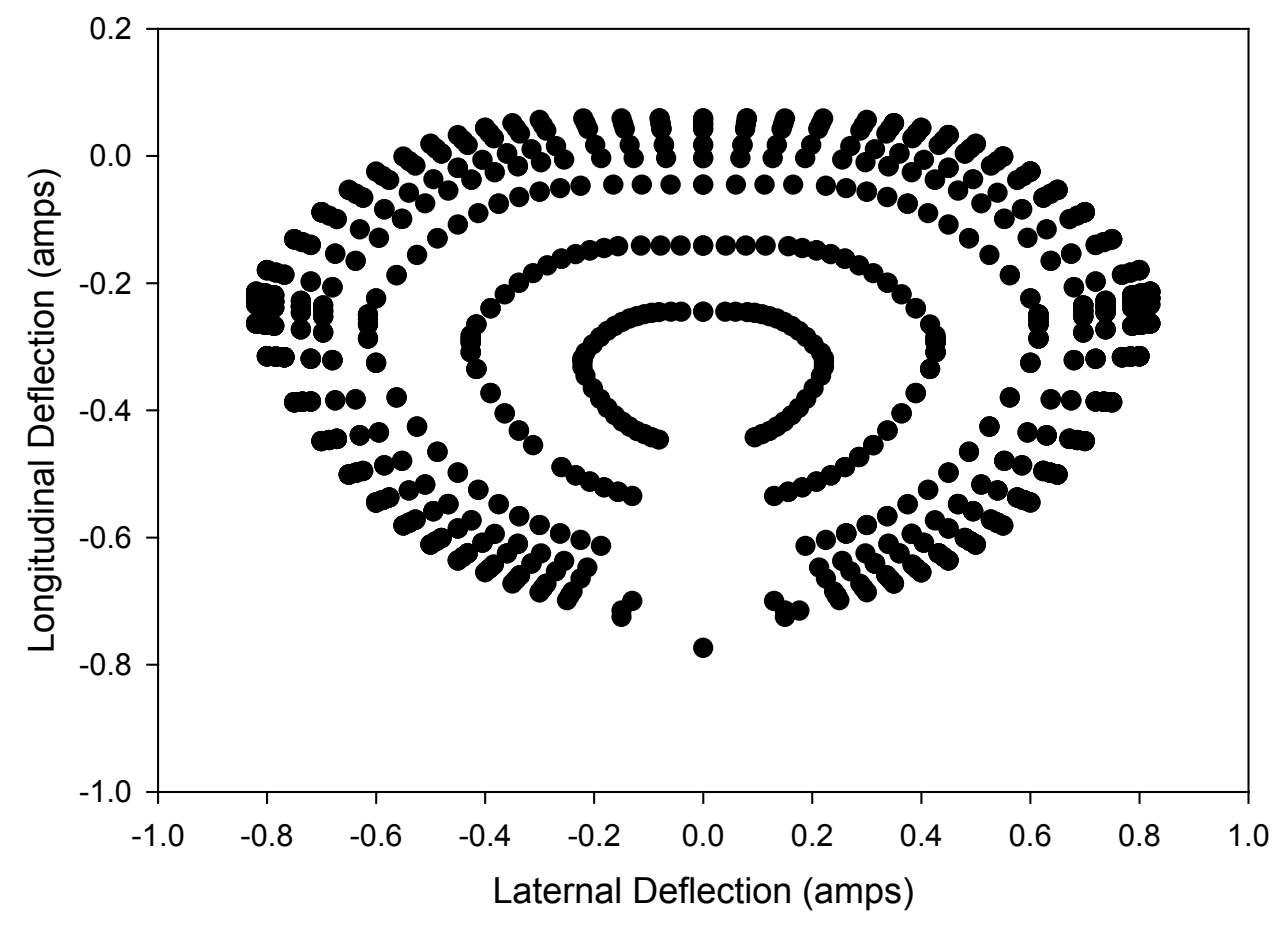

Figure 3.3.5 
All eight tracks were unnecessary and only track one (outer most track), track 3, and track 6 were kept and loaded into the electron guns sweeper. Using the handheld sweep adjuster, which allowed in-situ adjustments of $\mathrm{x}$ and $\mathrm{y}$ amplitudes and center positions, this pattern was fit to the deposition chamber and gave acceptable results but would occasionally drill through the source material in the back of the pocket if the material was not densely packed. Adjustments to the beam forming magnets inside the electron gun itself decreased the focus of the beam towards the back of the pocket and reduced the amount of drilling even further.

\section{- $\quad$ Section 3.3 \\ Aluminum Oxide from Electron-Beam Depositions}

These layers were where the light would be guiding and the ability of the layers to do so was another characterization criterion that was looked at. These depositions were initially performed on high pressure oxide (HIPOX) wafers which were cleaned in a five minutes acetone and five minutes methanol degreasing. In an attempt to achieve an understanding of the deposition parameters that were affecting the growths we performed numerous depositions under different conditions. Some trends were clearly seen, such as the fact that pressure inside the chamber at the beginning of the deposition had an effect on the resulting index. A lower pressure would result in a longer mean free path and therefore denser films. 


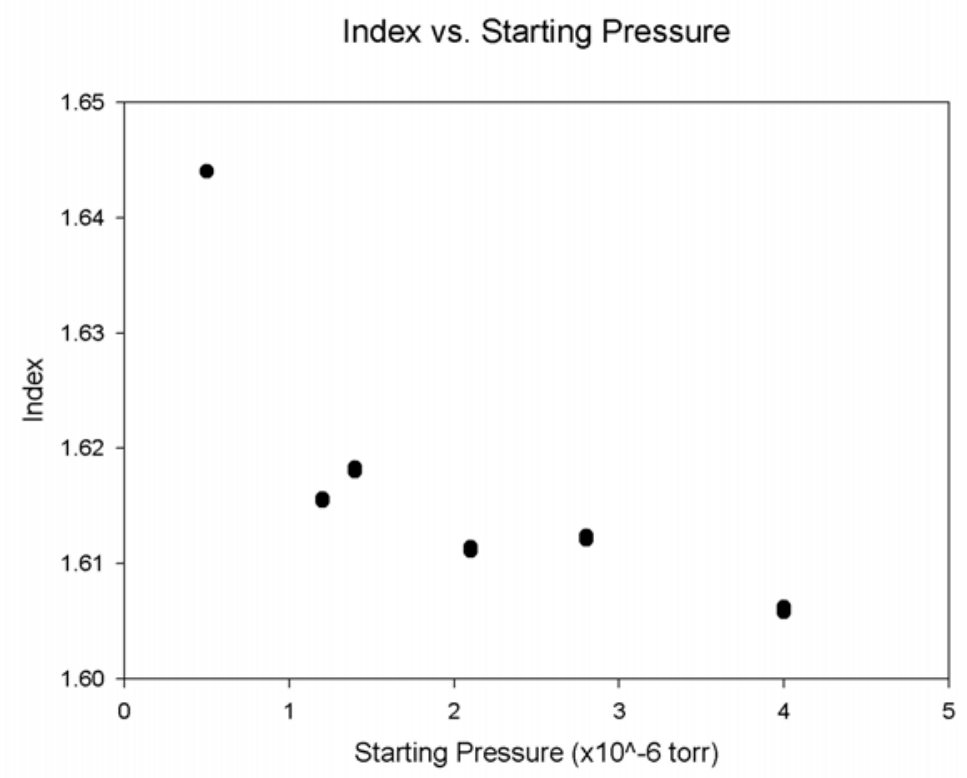

Figure 3.3.1

This trend was later found to be irreproducible, although in general a lower pressure would result in a higher index it appeared there were numerous parameters affecting the $\mathrm{Al}_{2} \mathrm{O}_{3}$ depositions. A study of seven samples grown during the same time period with pressure between $2.0-3.0 \times 10^{-6}$ torr, deposition rate of $10 \AA / \mathrm{s}$, and substrate rotation of 20 RPM showed a high standard deviation in the refractive indices amongst the films.

\begin{tabular}{|c|c|}
\hline Sample & Index of Refraction @ 633nm \\
\hline 127 & 1.6058 \\
\hline 129 & 1.6121 \\
\hline 131 & 1.6184 \\
\hline 133 & 1.6111 \\
\hline 135 & 1.6156 \\
\hline 143 & 1.6461 \\
\hline 150 & 1.6388 \\
\hline Std. Dev. & 0.0152 \\
\hline
\end{tabular}

Table 3.3.1 
Samples 127 through 135 fell in the region expected based on the previous graph. For unknown reasons some samples, such as 143 and 150, had drastically different indices even though growth conditions were believed to have remained consistent.

When placed in the prism coupler an $\mathrm{Al}_{2} \mathrm{O}_{3}$ film that guided well would show a streak visible across the entire surface of the substrate.

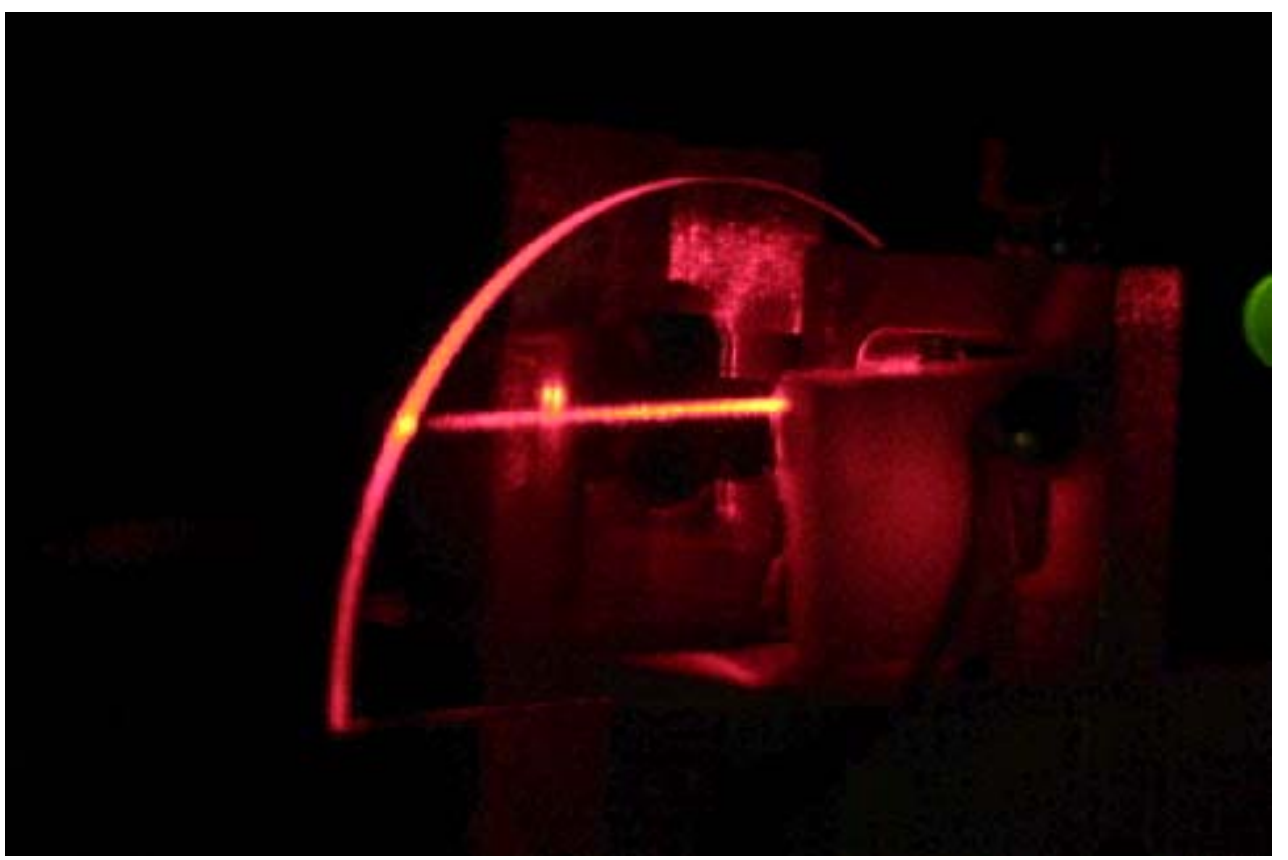

Figure 3.3.2

No scattering could be seen from contaminates inside the film and the losses appeared to be small. Unfortunately most of the films produced would not appear like this. Many were weakly guiding or had a large amount of scattering. 


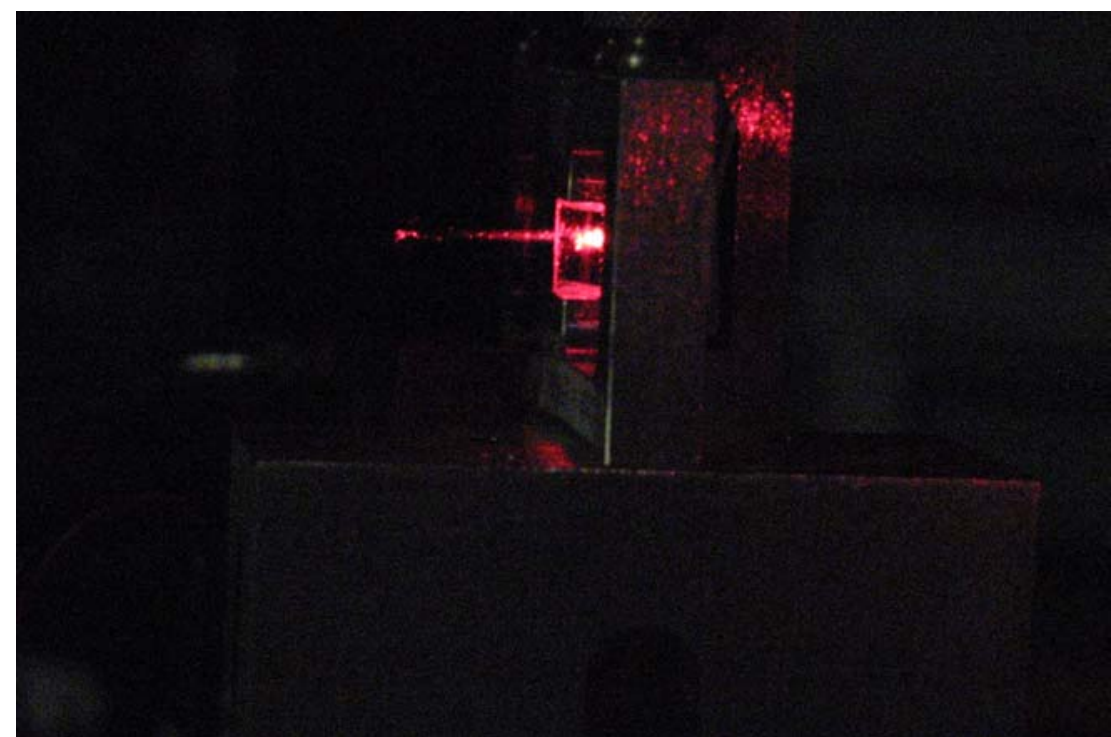

Figure 3.3.3

Slight Guiding

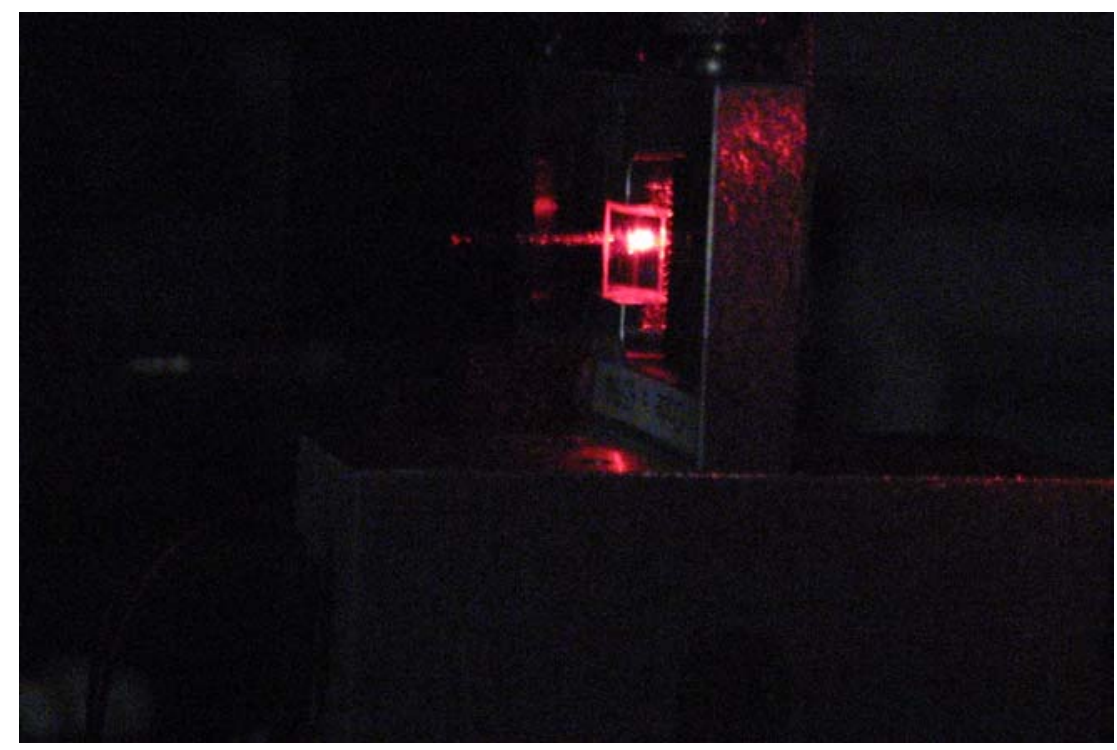

Figure 3.3.4

Slight Guiding 


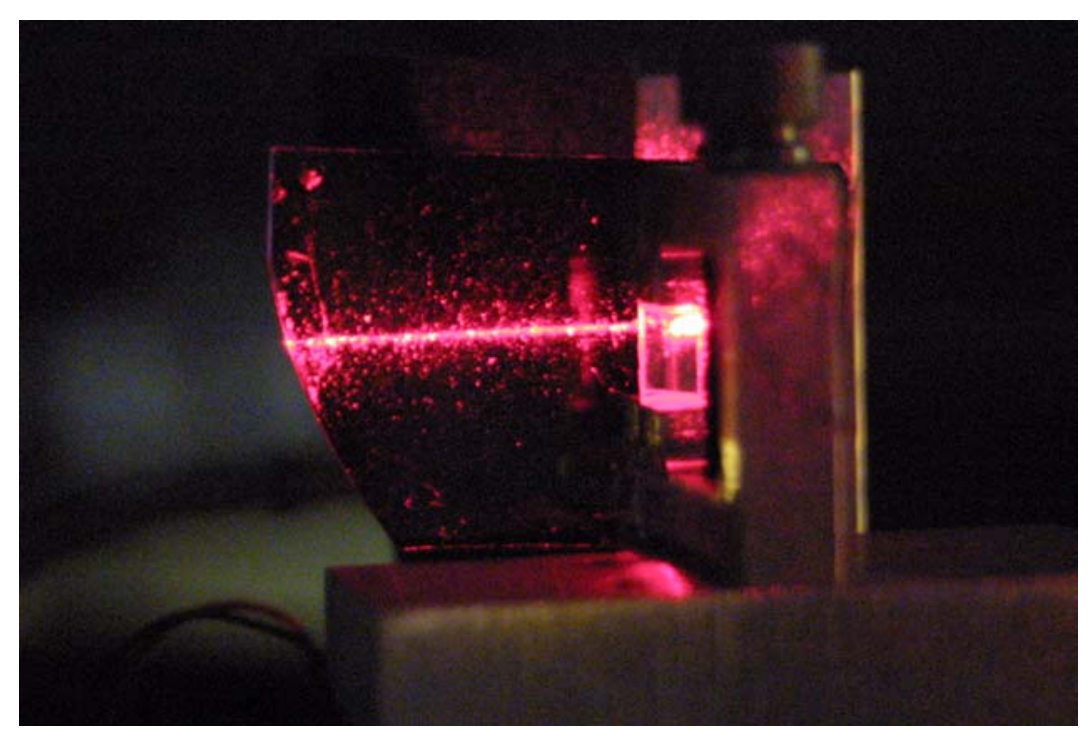

Figure 3.3.5

Guided well but with scattering

It was rarely possible to see any streak coming from the prism. Parameters such as chamber pressure, growth rate, source material age, amount of material on chamber walls, and the relative humidity of the day the sample was loaded were all considered possibilities for why some samples guided and some didn't. When no trend could be found an attempt was made to see if any contamination was present in the films. We believed that it was possible that carbon from the graphite liners or gold deposited on the chamber walls from previous depositions was entering into the films. Energy Dispersive Spectroscopy (EDS) was performed on a sample to see if any contamination was present. 


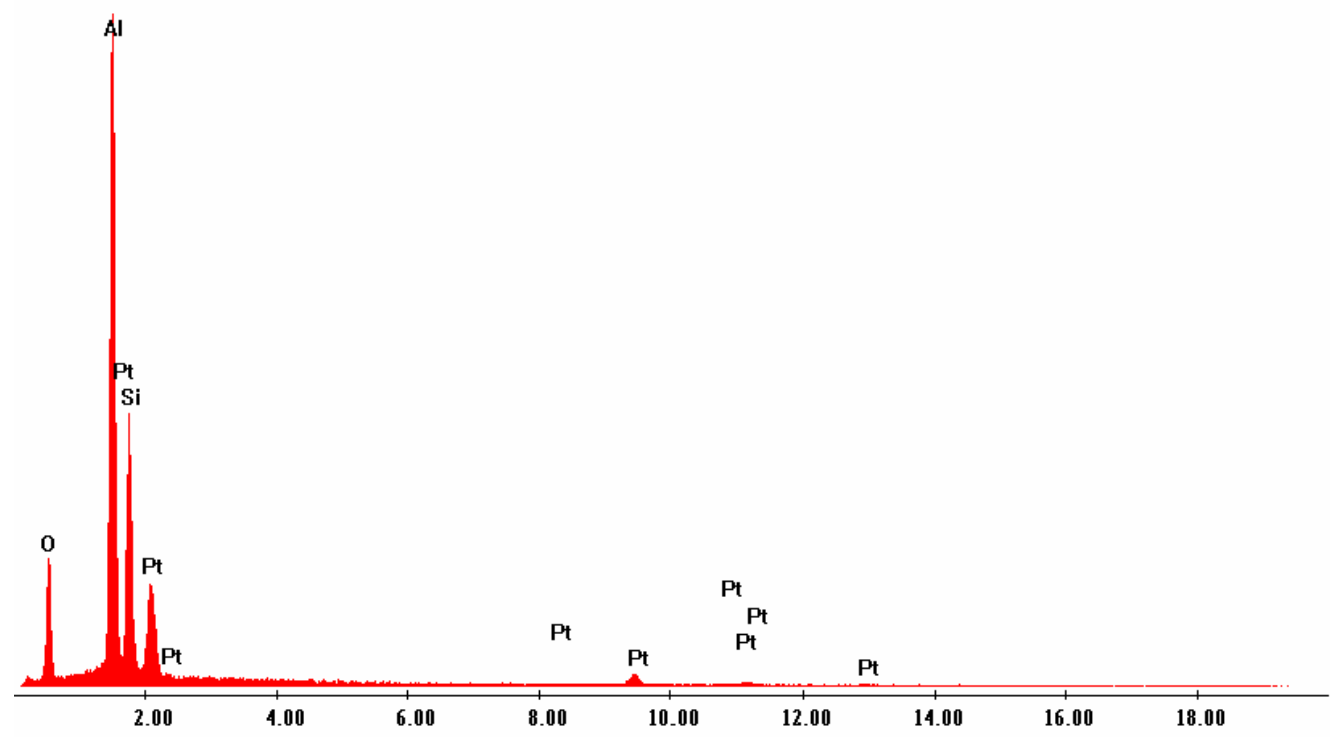

Figure 3.3.6

The platinum used to prepare the sample for EDS could clearly be seen as well as the silicon from the substrate, negligible carbon and no gold was detected.

\section{- $\quad$ Section 3.3.1 \\ Aluminum Oxide Depositions with Substrate Heat}

When the ability to perform depositions with substrate heat was added to the system new depositions were performed to characterize the system and attempt to bring greater index reproducibility and increased guiding by the samples. Using a selection of samples deposited on degreased borofloat glass wafers at pressures between $2.3-2.9 \mathrm{x}$ $10^{-6}$ torr, $290^{\circ} \mathrm{C}$ substrate temperature, and a deposition rate of approximately $10 \AA / \mathrm{s}$ we saw a decrease in the standard deviation in refractive indices when compared to those performed without substrate heat. 


\begin{tabular}{|l|l|}
\hline Sample & Index @ 633nm \\
\hline 243 & 1.6599 \\
\hline 251 & 1.6519 \\
\hline 254 & 1.6522 \\
\hline 249 & 1.6539 \\
\hline Std. Dev. & 0.0037 \\
\hline
\end{tabular}

Table 3.3.2

Comparing these samples with those without the substrate heater we saw that the mean index of refraction for samples had increased from 1.6211 to 1.6545. On occasion samples produced with deposition parameters thought to be exactly the same as the samples listed above would produce indices considerable different from the mean, such as sample 242 which had an index of refraction of 1.6924. No explanation could be found for why this was occurring.

With the addition of the thermocouple that was installed with the substrate heater it was possible to monitor chamber temperatures during a deposition without the use of substrate heat. During a typical eight minute deposition of $\mathrm{Al}_{2} \mathrm{O}_{3}$ material the temperature of the substrate would rise to over $200^{\circ} \mathrm{C}$ due to radiation from the hot source material. The effect on depositions that the substrate heater was having on the new samples was also occurring in samples without substrate but not until well into the deposition. Ellipsometric graded index measurements on a sample with and without substrate heat showed the effect. 
Effect of Heat on Depositions

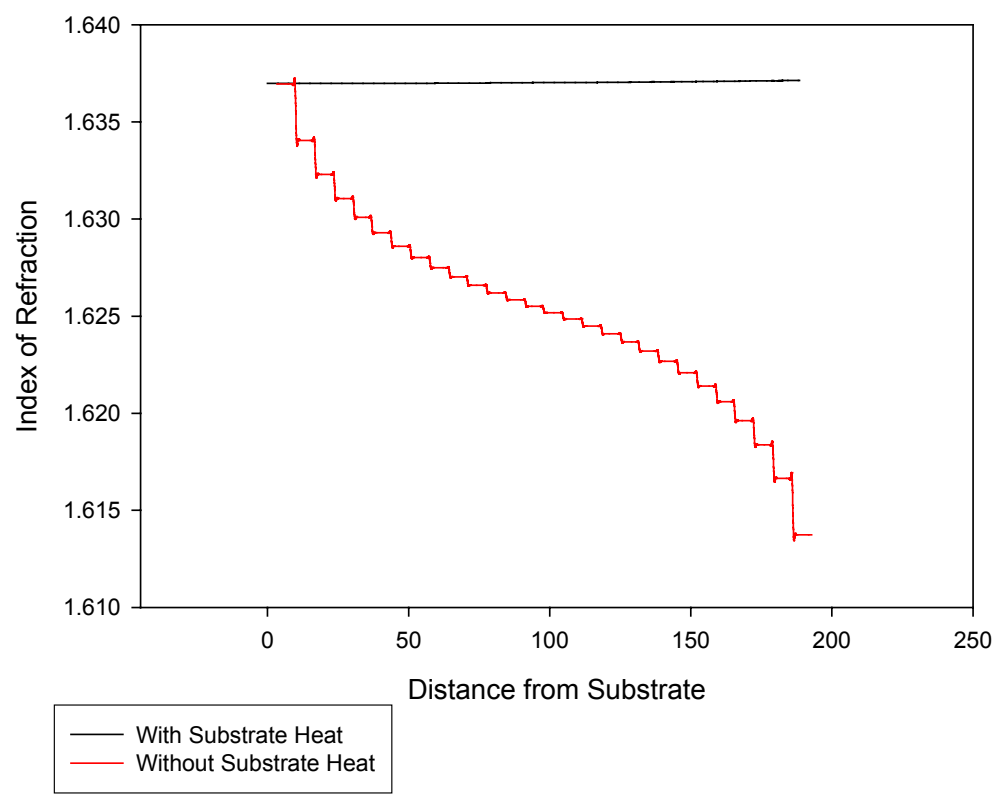

Figure 3.3.7

Although the effect of heat on the deposition could clearly be seen, it was puzzling that the index of refraction of the material was going lower as the substrate temperature rose. This was counterintuitive since the substrate heat would create a denser material with a higher index of refraction. This could be explained by outgassing of contaminates from the chamber walls as the chamber heated up or that the morphology of the film at low temperatures promoted porous film growth throughout the deposition.

The effect of substrate heat on the guiding of the $\mathrm{Al}_{2} \mathrm{O}_{3}$ films was poor. For all the aluminum oxide samples that were produced with substrate heat (samples 202 to 260) only two were considered to "guide well." The fact that the number of samples that guided with substrate heat was lower than the percentage that guided without the substrate heater it was unlikely the inability of the films to guide was due solely to the density of the films. Having previously ruled out contamination as a possibility it now seemed that the loss of an oxygen atom from the $\mathrm{Al}_{2} \mathrm{O}_{3}$ molecules was the most likely cause of the poor guiding. EDS on a sample grown with the substrate heat showed a trend of less oxygen and more aluminum with greater incident electron energy (corresponds to a greater penetration depth). 


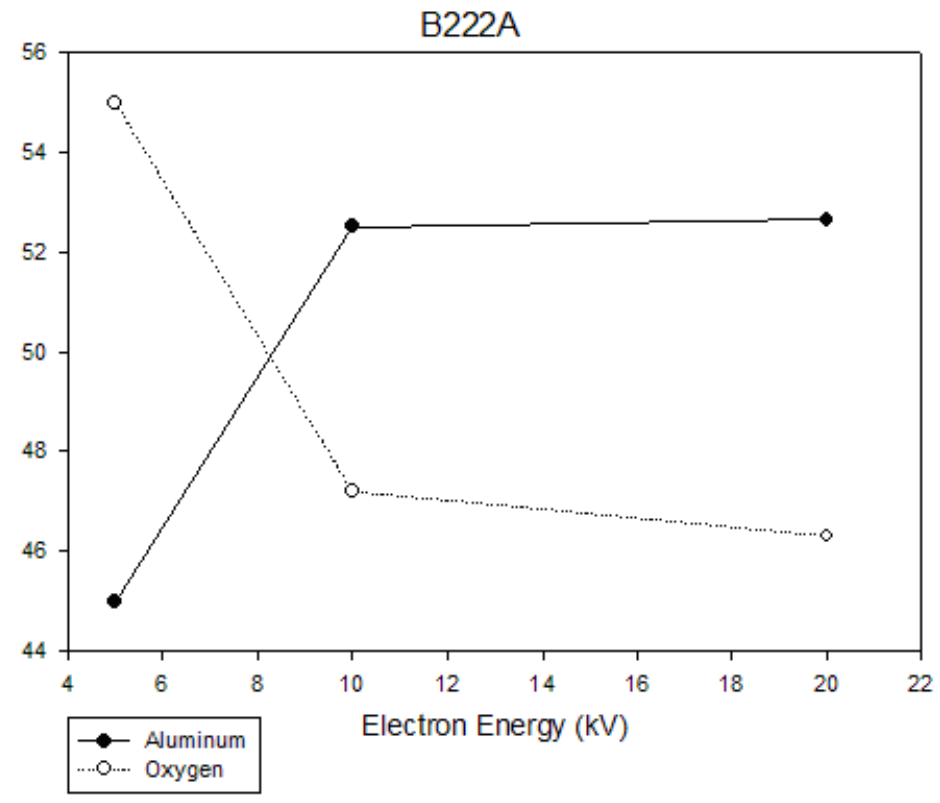

Figure 3.3.8

\section{- Section 3.3.2}

\section{Aluminum Oxide Depositions with Ion Beam Assist}

The improvements on the $\mathrm{Al}_{2} \mathrm{O}_{3}$ films with IBAD were evident with the first several samples produced. A streak was clearly seen for every $\mathrm{Al}_{2} \mathrm{O}_{3}$ film deposited which was thick enough to include a guiding mode.

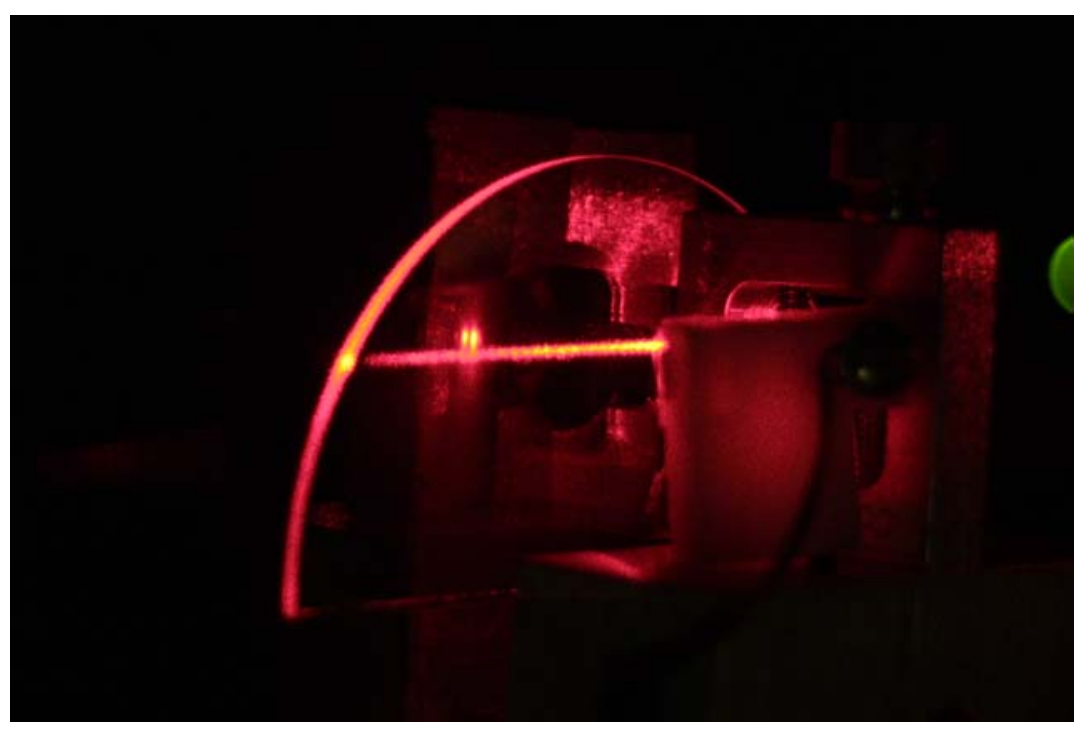

Figure 3.3.9

Sample 263 
Regardless of the operating parameters of the ion source the $\mathrm{Al}_{2} \mathrm{O}_{3}$ film would guide. It was not possible to visually detect a difference in the losses of the films when comparing one set growth parameters to another. Research now focused on characterization of the ion source to find the operating parameters that would give the most consistent index with the greatest amount of moisture stability.

These experiments were performed on borofloat wafers which were soaked and sonicated in acetone for five minutes and then soaked in methanol for five minutes. Samples were loaded into the chamber and the substrate heater was set to $290^{\circ} \mathrm{C}$ for one hour. After one hour the substrate heater was turned off and the chamber continued to pump down for an additional two hours. After this time the substrate heat was turned back on and set to $200^{\circ} \mathrm{C}$ which it reached in about seven minutes. At this point pressures were between 6.0 to $6.2 \times 10^{-6}$ torr. An oxygen flow rate of $17.5 \mathrm{sccm}$ was set and neutralizer current was set to 23.0 amps. The drive current was varied among the experiments (between 1.25 to $2.5 \mathrm{amps}$ ) to see what range of refractive indices the system was capable of achieving. After thirty seconds to one minute the chamber pressure would reach equilibrium around $8.2-8.6 \times 10^{-5}$ torr and the depositions would begin. Under computer control the electron-gun would soak the source material for thirty seconds at $22 \%$ power $(1.3 \mathrm{~kW})$ and then lower to $17 \%$ power $(1.0 \mathrm{~kW})$ to soak for another thirty seconds. The shutter would then open and the deposition rate would be 3.5 $\pm 1.5 \AA / \mathrm{s}$. The system would automatically adjust the emission current from the electron gun to maintain a $3.5 \pm 0.2 \AA / \mathrm{s}$ deposition rate. 


\section{Depositied Alumina Film Indices Based on Various Drive Currents}

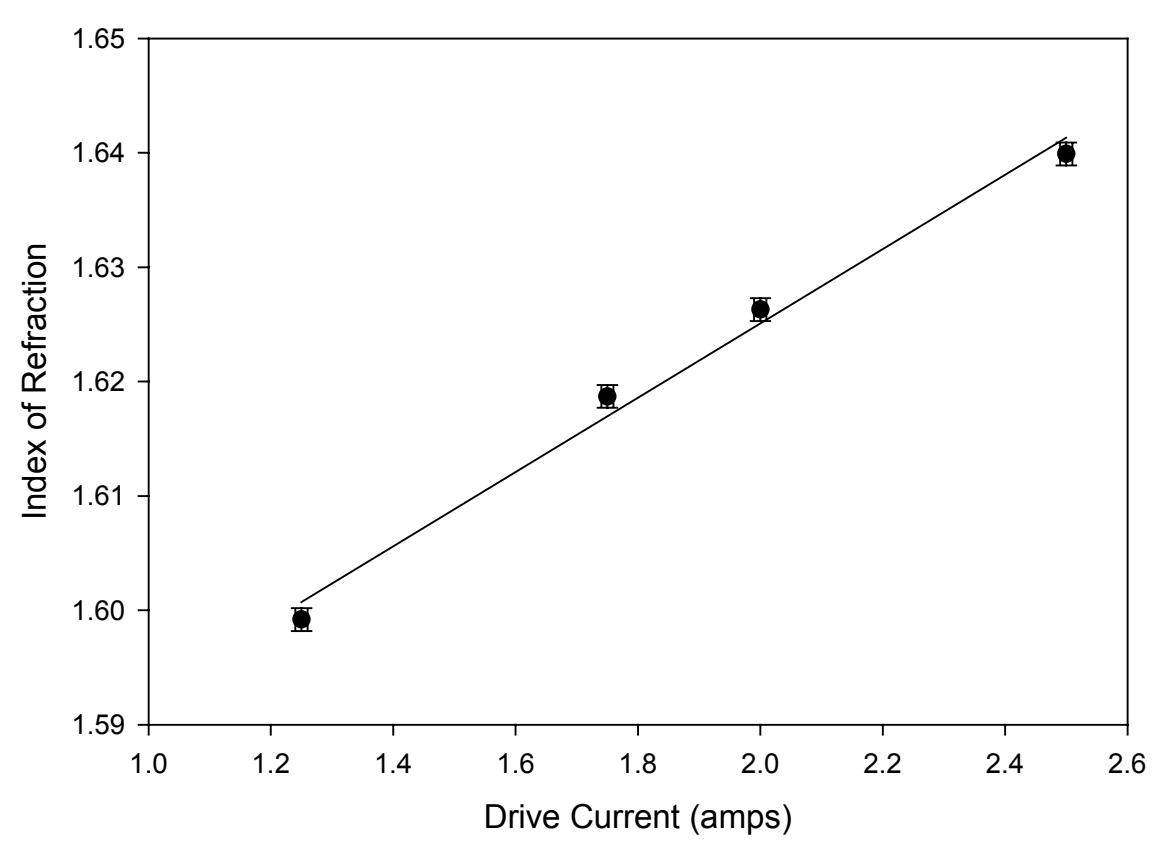

Figure 3.3.10

The typical index refraction for aluminum oxide is around 1.63 at $633 \mathrm{~nm}$, therefore a drive current of 2.25 amps was chosen as the optimal value for future depositions. Several single layer films were produced with the previous deposition parameters but now holding the drive current to 2.25 amps. Before the beginning of the experiment the e-beam chamber was cleaned and new source material was loaded. Before each new deposition small amounts of source material would be added to replenish what had been used. 


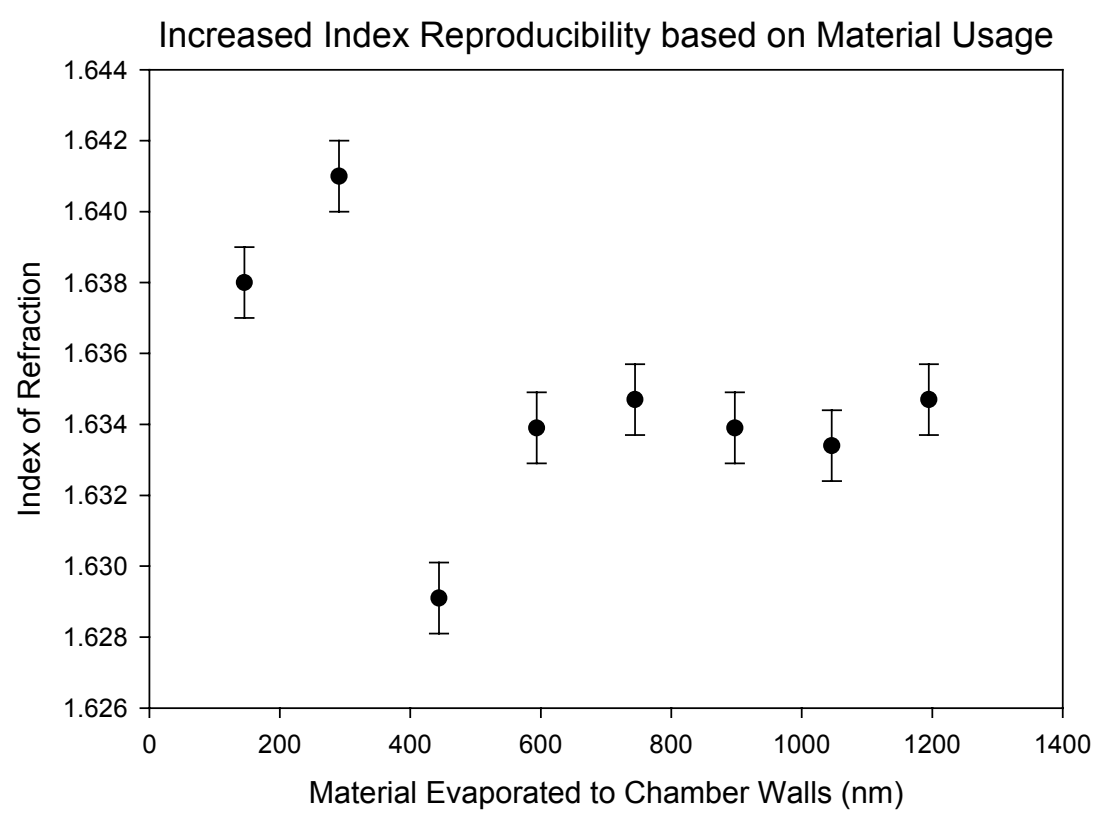

Figure 3.3.11

The first three samples produced showed large amounts of standard deviation in the refractive indices, $\sigma=0.0062$, while the next five samples showed a significant decrease in standard deviation to $\sigma=0.00057$. This result proved that chamber wall and source material conditioning were important for reproducible results and that source material should not be replaced with every deposition but only replenished as needed.

\begin{tabular}{|l|l|}
\hline Sample & Index \\
\hline 292 & 1.63390 \\
\hline 293 & 1.63470 \\
\hline 294 & 1.63390 \\
\hline 295 & 1.63340 \\
\hline 296 & 1.63470 \\
\hline Std. Dev & 0.00057 \\
\hline
\end{tabular}

Table 3.3.3 


\section{- Section 3.3.3}

\section{Aluminum Oxide Moisture Stability}

The biosensor device will be working in an aqueous solution and the moisture stability of the samples was of considerable concern. The stability of the films, specifically the top aluminum oxide layer, depends on how much water can enter into the pours of the films and the population of - $\mathrm{OH}$ groups; films with higher indices should be denser, have less film surface area, and therefore show less of a change when exposed to water $[22,23]$. To test how the films changed when exposed to water several growths were performed at several different ion drive currents. Cleaned borofloat samples were loaded into the chamber and heated to $275^{\circ} \mathrm{C}$ for five minutes. After this the heat was turned off and the chamber pumped down for three hours. After the pump down the substrate heat was turned back on to $200^{\circ} \mathrm{C}$ and the oxygen flow rate was set to 17.5 sccm; the pressure would be between $8.7-9.1 \times 10^{-6}$ torr. Substrate rotation was set to 20 RPM and depositions occurred to a thickness of $140 \mathrm{~nm}$ and at rate of $3.5 \pm 0.2 \AA / \mathrm{s}$.

After completion the chamber was allowed to cool for over two hours so the temperatures would reach below $75^{\circ} \mathrm{C}$. When removed the samples were taken to the ellipsometer and thickness and refractive index measurements were taken. The samples would be soaked in deionized water for 20 minutes and dried just enough to remove any remaining water from the surface. Ellipsometry measures were again taken. 


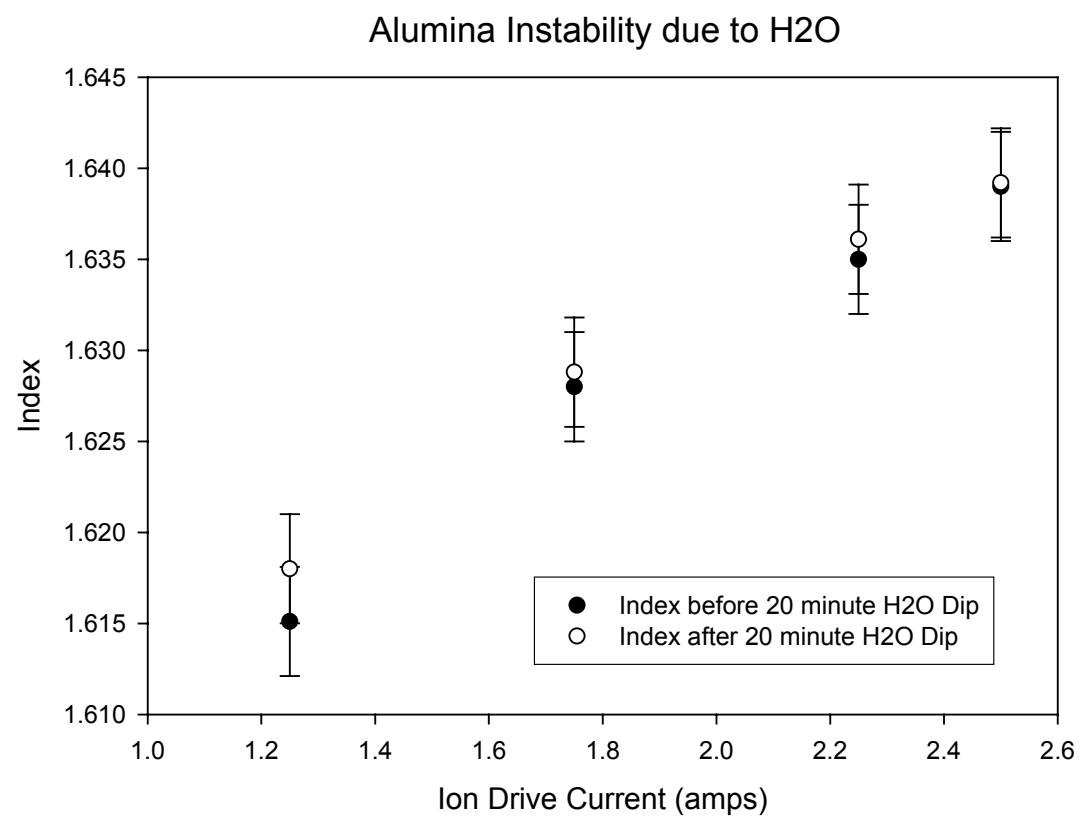

Figure 3.3.12

Index Shift after 20 minute Deionized Water Soak

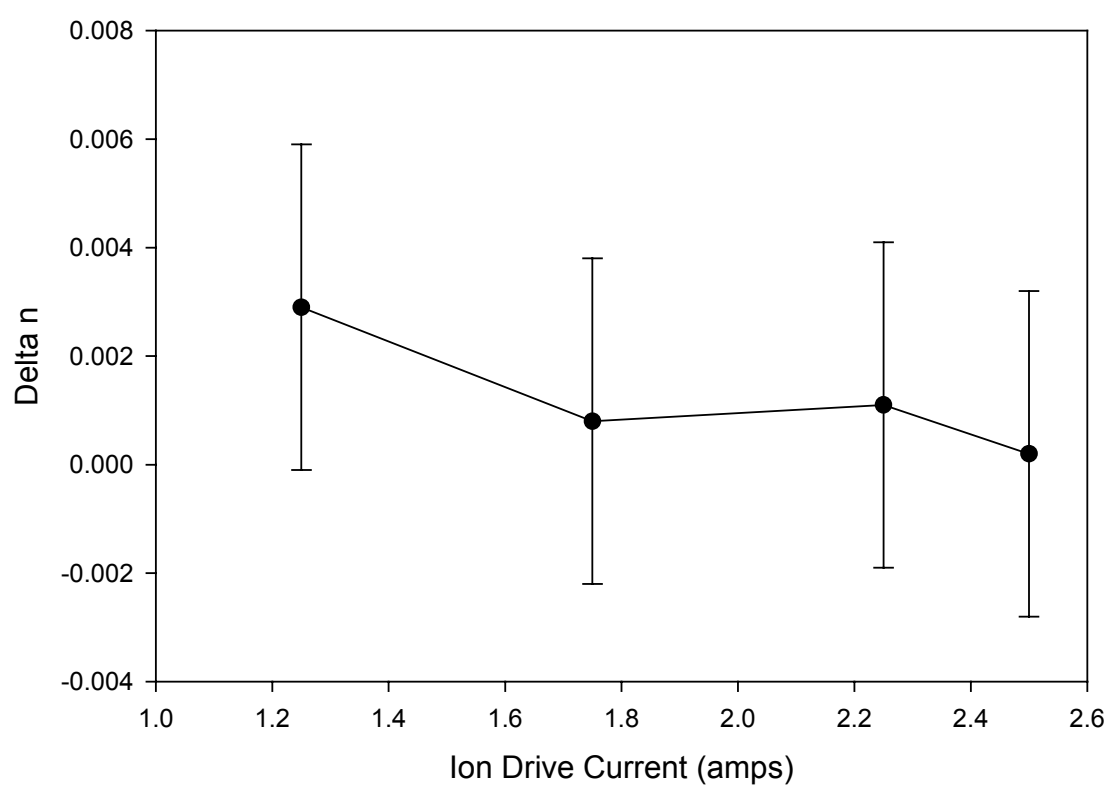

Figure 3.3.13

As expected the increased drive current produced films with higher indices due to the higher densities. Although the $\Delta \mathrm{n}$ 's were most likely within the limits of the ellipsometer, measurements consistently showed a change refractive index after the water 
soak and a decrease in $\Delta \mathrm{n}$ with higher drive currents. Although aluminum oxide is considered to be one of the most moisture stable dielectrics it was unexpected that so little of a change was seen in the samples grown at lower drive currents. It was possibly that the water was leaving the film quickly and that very little was left once the sample's surface had dried and ellipsometer measurements were taken the second time.

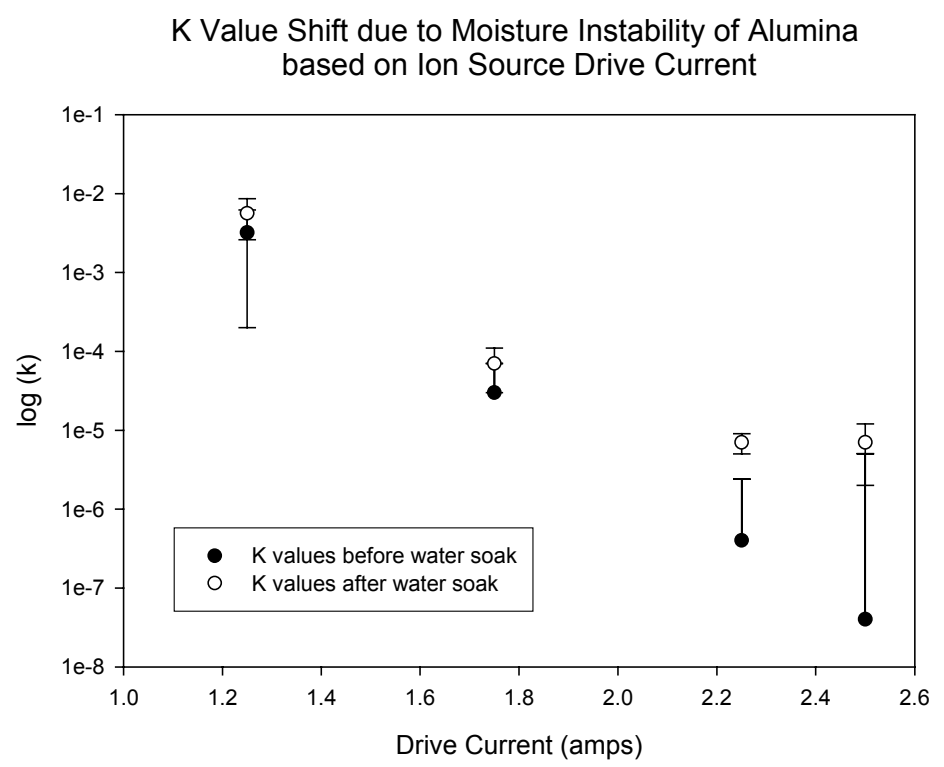

Figure 3.3.14

Delta k based on lon Source Drive Current

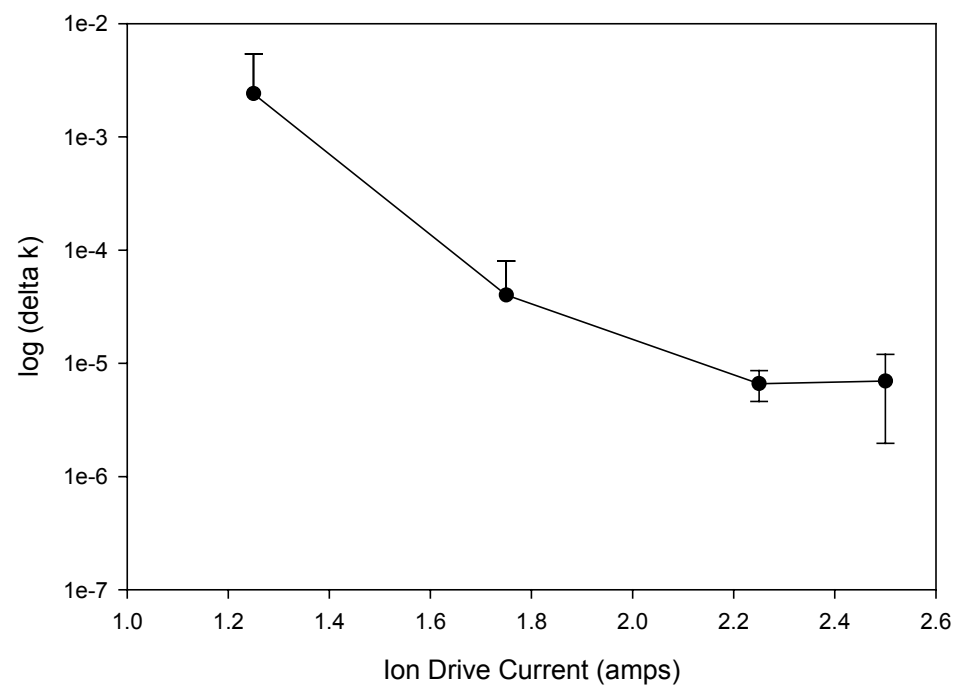

Figure 3.3.15 
Again the data shows what was expected from films with higher drive currents. The $\mathrm{k}$ values $(\widetilde{n}=n+i k)$ were decreasing with higher energies while the $\Delta \mathrm{k}$ 's were also becoming smaller.

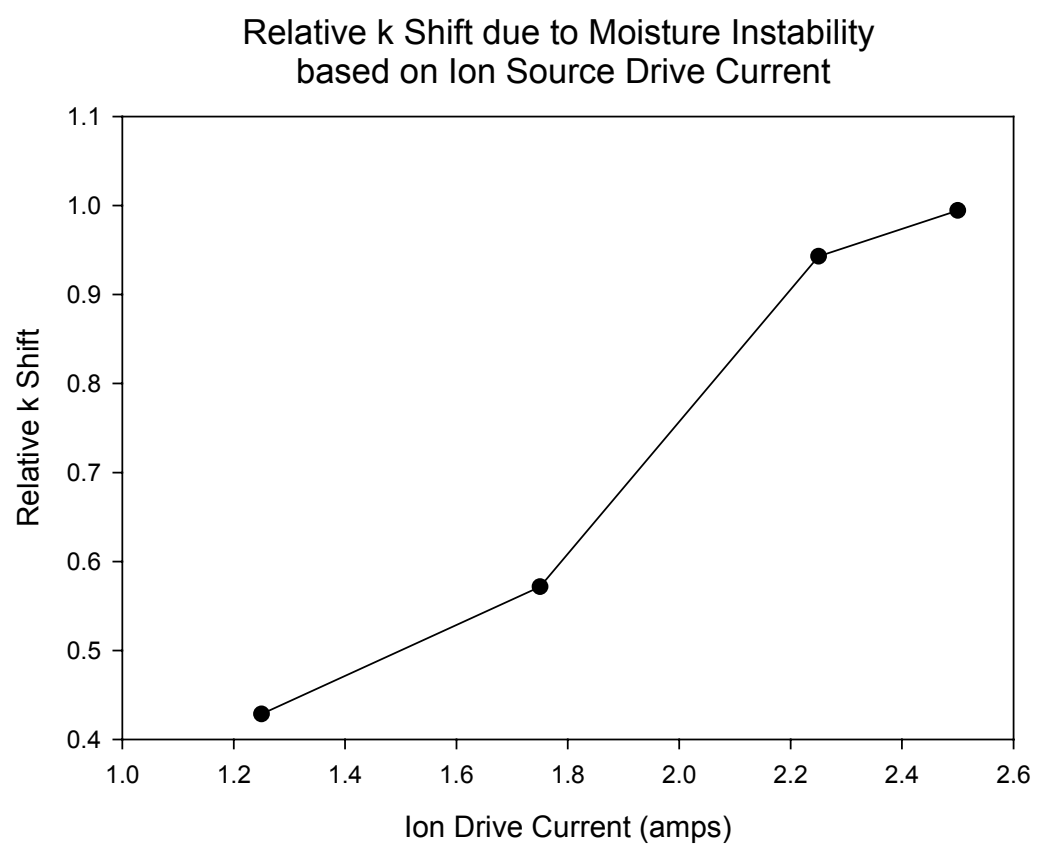

Figure 3.3.16

The relative shifts $\left(\frac{\text { initial }- \text { final }}{\text { initial }}\right)$ were seen to be increasing with drive current, $\mathrm{k}$ values were increasingly sensitive to the presence of water when losses reached minimal values in the high quality alumina films.

- Section 3.4

\section{Structure Growth}

Having resolved many of the chemical and optical issues involved with the depositions the research focused on growing the waveguide structures with the greatest amount of thickness control possible. 


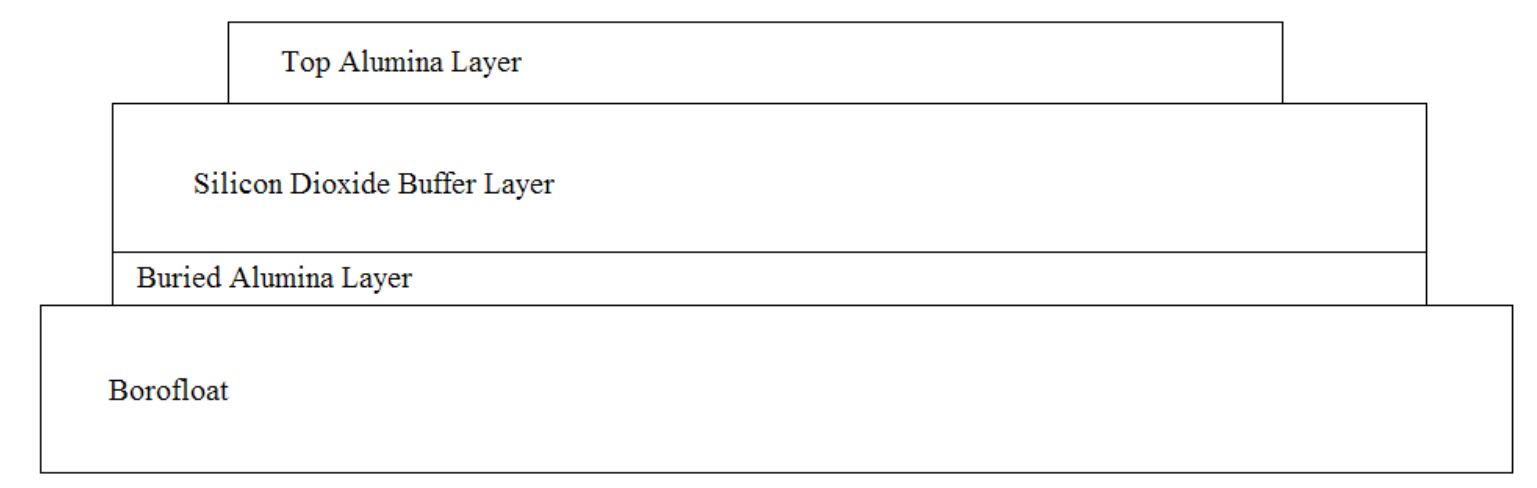

Figure 3.4.1

\section{- Section 3.4.1}

\section{Thickness Control Measurements}

To test the limits of the quartz crystal deposition monitor eight depositions were performed. These samples were on borofloat wafers that were soaked in acetone and sonicated for five minutes then soaked in methanol for five minutes. Samples were loaded in the chamber and after the cryopump was opened the substrate heat was turned on and allowed to reach $290^{\circ} \mathrm{C}$. After one hour at $290^{\circ} \mathrm{C}$ the substrate heat was lowered to $260^{\circ} \mathrm{C}$ and the ion source was started with an oxygen flow of $17.5 \mathrm{sccm}$, neutralizer current of $23.0 \mathrm{amps}$, and drive current of $2.25 \mathrm{amps}$. After allowing one to two minutes for the pressure to stabilize the deposition process started. The material was melted in with a soak power of $22 \%(1.3 \mathrm{~kW})$ for thirty seconds and then a soak power of $17 \%$ $(1.0 \mathrm{~kW})$ for thirty more seconds. After completion the shutter opened and the deposition monitor controlled the emission current to maintain a $3.5 \pm 0.2 \AA / \mathrm{s}$ deposition rate until a thickness of $144 \mathrm{~nm}$ had been reached. 
Ellipsometer Measured Thickness of 144nm Deposition Based on Crystal Usage

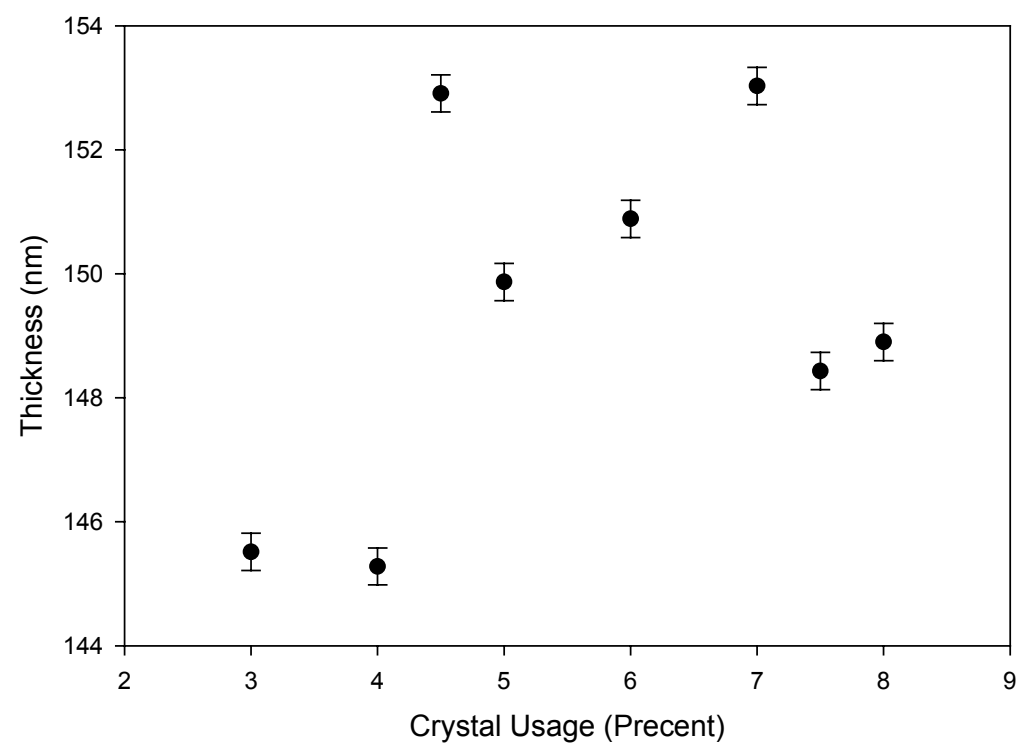

Figure 3.4.2

The first two runs performed using the new crystals were within $0.3 \mathrm{~nm}$ of each other $(145.52 \mathrm{~nm}$ and $145.28 \mathrm{~nm})$ and were within the error bars for the ellipsometer. If this accuracy continued then retooling the crystal monitor would mean future depositions would be within $0.3 \mathrm{~nm}$ of $144 \mathrm{~nm}$. The data showed that after the second deposition the accuracy of the crystal monitor degraded and the eight samples had a standard deviation of $2.95 \mathrm{~nm}$.

Although the crystal monitor closed the deposition shutter at $144 \mathrm{~nm}$ the display continued to fluctuate for several minutes after deposition. This did not result from any deposition or flaking of material onto/off of the crystal but due to stress change, absorption of vapor, or change in vibrational modes of the crystal during cool down. For this reason it was imperative that the temperature of the crystal monitor stay constant during deposition.

The thickness reported by the crystal monitor at the end of the deposition increased (after cool down) with each deposition. 


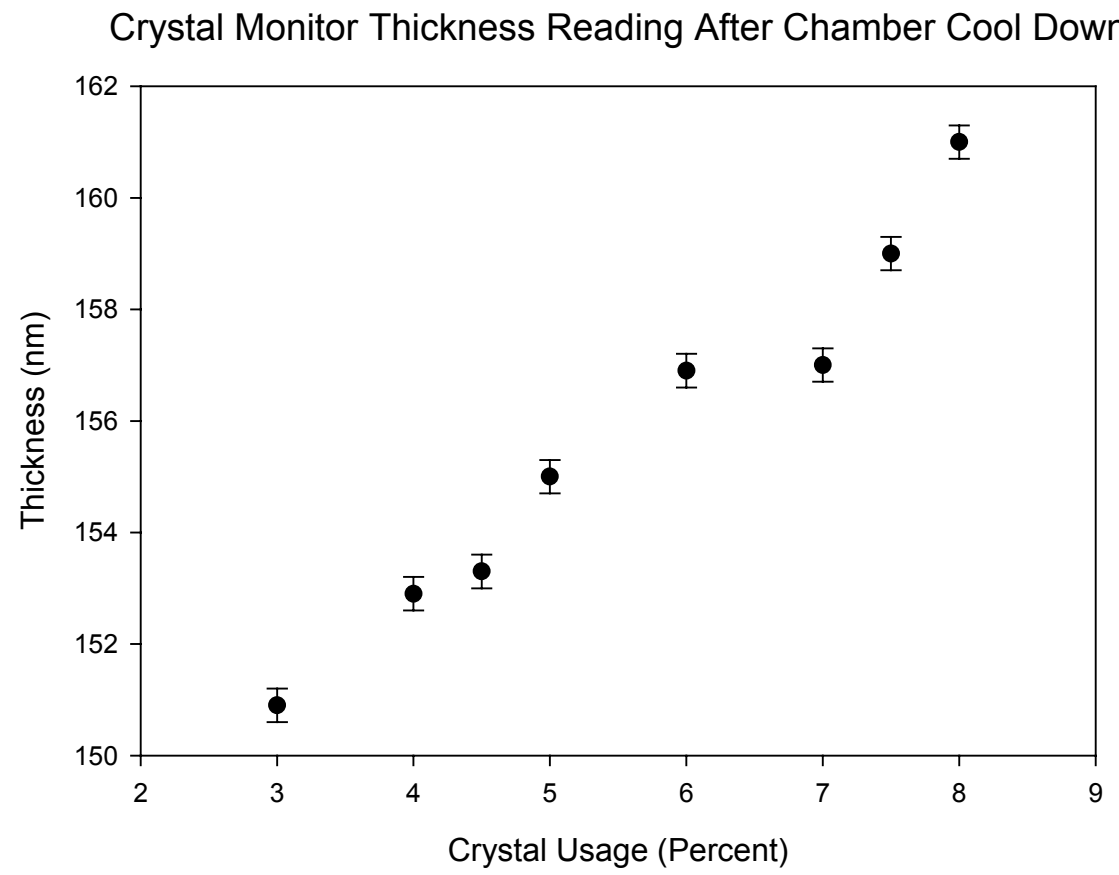

Figure 3.4.3

This was a good indicator that there was a problem with the crystal monitor's ability to adapt to the increase in heat it was experiencing during depositions. Since the ion source and substrate heaters were running for several minutes before the depositions started the most likely cause was the sudden surge of radiation the crystal experienced as soon as the deposition shutter was opened. To test this, a run was performed in which the electron gun current was high enough to turn the aluminum oxide molten but too low to vaporize the material. The crystal used had one previous deposition on it, $170 \mathrm{~nm}$ of $\mathrm{Al}_{2} \mathrm{O}_{3}$. When the shutter opened the crystal monitor should not have reported any change. 


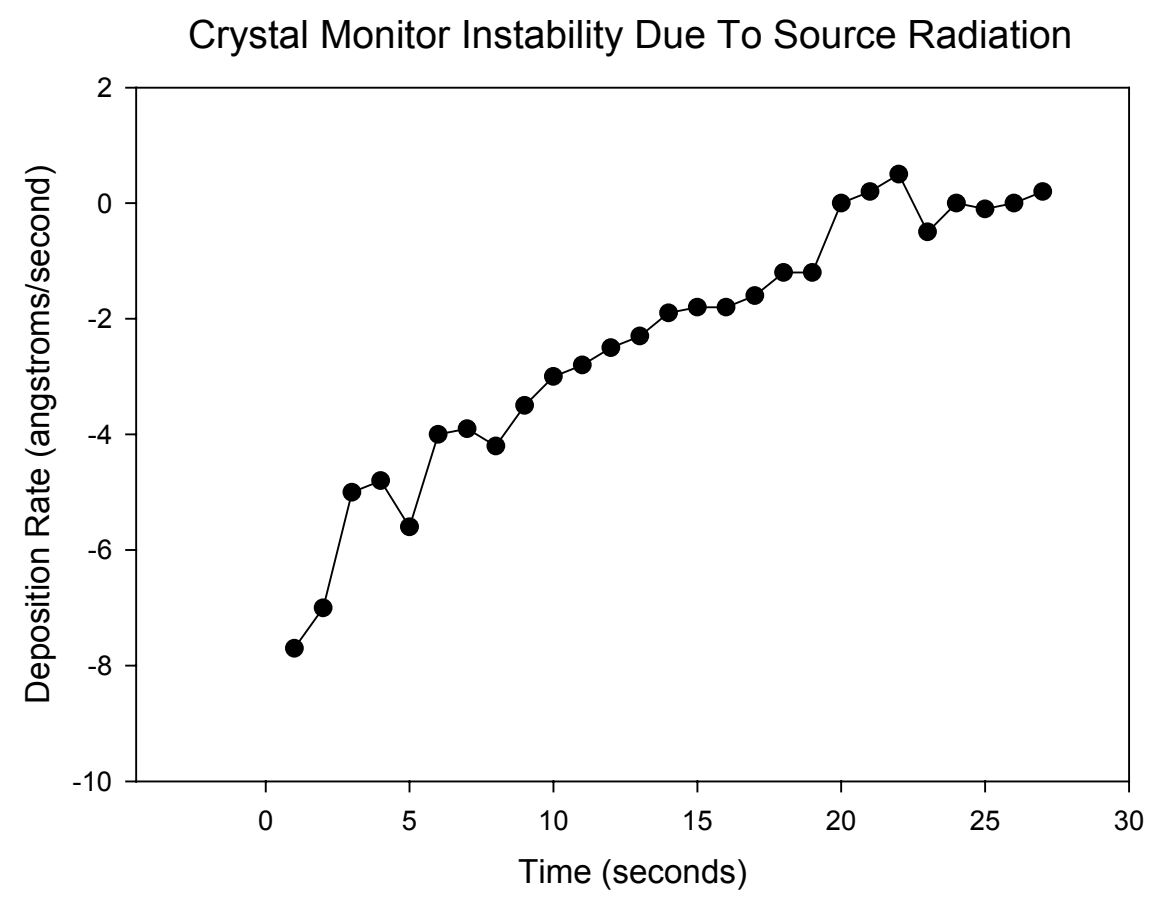

Figure 3.4.4

During this period the crystal monitor reported a deposition of $\approx-6.5 \mathrm{~nm}$. This meant that there was probably some absolute offset that occurs for all depositions due to radiation surge from the source material.

These results supported the idea that a new crystal would give better results than a used one but the effect was still evident for unused crystals. A quick drop in the deposition rate could be seen between the time the shutter opened and the source material reached the crystal indicating the same desorption of vapor was occurring from the crystal surface. In addition new crystals showed a steady increase in deposited material (around $0.2-0.8 \AA / \mathrm{s}$ ) between the time the ion source was started and the shutter was opened. This did not occur on any subsequent uses of the crystal and was almost certainly the result of the oxidation of the crystal's silver coating. It was not known how this might have affected the monitor's accuracy but the crystals do have a shelf life of one year due to the eventual oxidation of their surface. 


\section{- Section 3.4.2}

\section{Ellipsometer, Prism Coupler, and Simulation Results}

It becomes increasing difficult to accurately measuring the refractive indices and thicknesses of films when multiple films are present. In an attempt to determine the accuracy of the ellipsometers' measurements of multiple layer structures the data that was collected from several structures was modeled in CAD software to determine the locations of the two guided modes that should occur in the structures. The actual locations were then determined by the use of a prism coupler.

$\begin{array}{ccccccccc}\begin{array}{c}\text { Buried Guide } \\ (\mathbf{n m})\end{array} & \begin{array}{c}\text { Oxide } \\ (\mathbf{n m})\end{array} & \begin{array}{c}\text { Top Guide } \\ (\mathbf{n m})\end{array} & \begin{array}{c}\text { PC } \\ \text { Mode 1 }\end{array} & \begin{array}{c}\text { PC } \\ \text { Mode 2 }\end{array} & \begin{array}{c}\text { Sim. } \\ \text { Mode 1 }\end{array} & \begin{array}{c}\text { Sim. } \\ \text { Mode 2 }\end{array} & \begin{array}{c}\text { Mode 1 } \\ \text { Error }\end{array} & \begin{array}{c}\text { Mode 2 } \\ \text { Error }\end{array} \\ 154.6 & 1082 & 193.8 & 1.4923 & 1.5159 & 1.4889 & 1.5111 & 0.0034 & 0.0048 \\ 154.8 & 1129.5 & 194.4 & 1.4905 & 1.5148 & 1.489 & 1.5112 & 0.0015 & 0.0036 \\ 177.3 & 1138.6 & 232.2 & 1.5122 & 1.5253 & 1.5059 & 1.519 & 0.0063 & 0.0063 \\ 170.7 & 1114.7 & 240.2 & 1.5179 & 1.5246 & 1.5059 & 1.5168 & 0.012 & 0.0078 \\ 196.1 & 990.4 & 263.8 & 1.5253 & 1.5308 & 1.5187 & 1.5256 & 0.0066 & 0.0052\end{array}$

Mode error, the difference between prism coupler actual locations and where simulations say the location should be based on the data collected from the ellipsometer, was within the error tolerances of the ellipsometer. This is due to the numerous factors that come into effect when multiple films are present, such as surface roughness and intermixes. Changing model options will produce changes in the values solved for by the ellipsometer. A typical result would involve measuring the first two layers thicknesses during the mask change before the top aluminum oxide deposition; after the completion of the third layer the values obtained are used as the base for the full model. Sample B327AOA showed a film stack with the parameters,

\begin{tabular}{|rlr|}
\hline 3 & b320a alumina opt_cnst & $193.805 \mathrm{~nm}$ \\
\hline 2 & cauchy & $1082.049 \mathrm{~nm}$ \\
\hline 1 & b320a alumina opt_cnst & $154.618 \mathrm{~nm}$ \\
\hline 0 & cauchy & $1 \mathrm{~mm}$
\end{tabular}

Figure 3.4.5 
an alumina index of 1.639, and a silicon dioxide index of 1.475. By changing the model so that the thicknesses of the two underlying layers could be solved for and was not set to values from a previous measurement the model now solves for the values

\begin{tabular}{|llr|}
\hline 3 & b320a alumina opt_cnst & $193.148 \mathrm{~nm}$ \\
\hline 2 & cauchy & $1083.587 \mathrm{~nm}$ \\
\hline 1 & b320a alumina opt_cnst & $153.898 \mathrm{~nm}$ \\
\hline 0 & cauchy & $1 \mathrm{~mm}$ \\
\hline
\end{tabular}

Figure 3.4.6

The multiple optical constants are not solved for at the same time. Those that were used for this model were from previous growths that were single layers on borofloat substrates. Allowing the model to adjust the optical constant of one layer still provides valid results; solving for $\mathrm{n}$ and $\mathrm{k}$ values of the top aluminum oxide layer changed solved values to

\begin{tabular}{|llr|}
\hline 3 & cauchy & $193.769 \mathrm{~nm}$ \\
\hline 2 & cauchy & $1083.395 \mathrm{~nm}$ \\
\hline 1 & b320a alumina opt_cnst & $154.237 \mathrm{~nm}$ \\
\hline 0 & cauchy & $1 \mathrm{~mm}$ \\
\hline
\end{tabular}

Figure 3.4.7

The top alumina layer now has an index of 1.635 as compared to 1.639 before.

It was also possible that some amount of intermix between any two layers was occurring. The model was adjusted to account for this.

\begin{tabular}{|llr|}
\hline 5 & cauchy & $194.280 \mathrm{~nm}$ \\
\hline 4 & Intermix & $13.891 \mathrm{~nm}$ \\
\hline 3 & cauchy & $1082.558 \mathrm{~nm}$ \\
\hline 2 & Intermix & $0.000 \mathrm{~nm}$ \\
\hline 1 & b320a alumina opt_cnst & $154.190 \mathrm{~nm}$ \\
\hline 0 & cauchy & $1 \mathrm{~mm}$
\end{tabular}

Figure 3.4.8

A $13.9 \mathrm{~nm}$ layer of material consisting of 50\% aluminum oxide and 50\% silicon dioxide was solved for between the top aluminum oxide and the silicon dioxide layer. No intermix was found between the bottom aluminum oxide and silicon dioxide layer. This result suggests some effect occurring due to the vacuum break. 
Adding surface roughness to the model allowed further adjustments, most notably an increase in the intermix to $17 \mathrm{~nm}$.

\begin{tabular}{|rlr|}
\hline 6 & srough & $0.955 \mathrm{~nm}$ \\
\hline 5 & cauchy & $194.721 \mathrm{~nm}$ \\
\hline 4 & Intermix & $17.555 \mathrm{~nm}$ \\
\hline 3 & cauchy & $1081.099 \mathrm{~nm}$ \\
\hline 2 & Intermix & $0.000 \mathrm{~nm}$ \\
\hline 1 & b320a alumina opt_cnst & $154.737 \mathrm{~nm}$ \\
\hline 0 & cauchy & $1 \mathrm{~mm}$ \\
\hline
\end{tabular}

Figure 3.4.9

None of these models were any less valid than any other. Work remains to be done to develop the correct model so that the most accurate thickness and refractive index measurements can be obtained.

\section{- Section 3.4.3}

\section{Surface Roughness}

Before the biolayer was grown on the deposited aluminum oxide films experiments were performed on crystalline aluminum oxide (sapphire) wafers. Initial results showed that the atomic smoothness of the sapphire wafers allowed the biolayer molecules to become more densely packed on the substrate surface as compared to an amorphous aluminum oxide film. Atomic Force Microscopy (AFM) images showed the root-mean-square roughness of the deposited films was around $2 \mathrm{~nm}$. Decreasing the surface roughness to the minimum value possible would provide the best conditions for the biolayer to grow. 


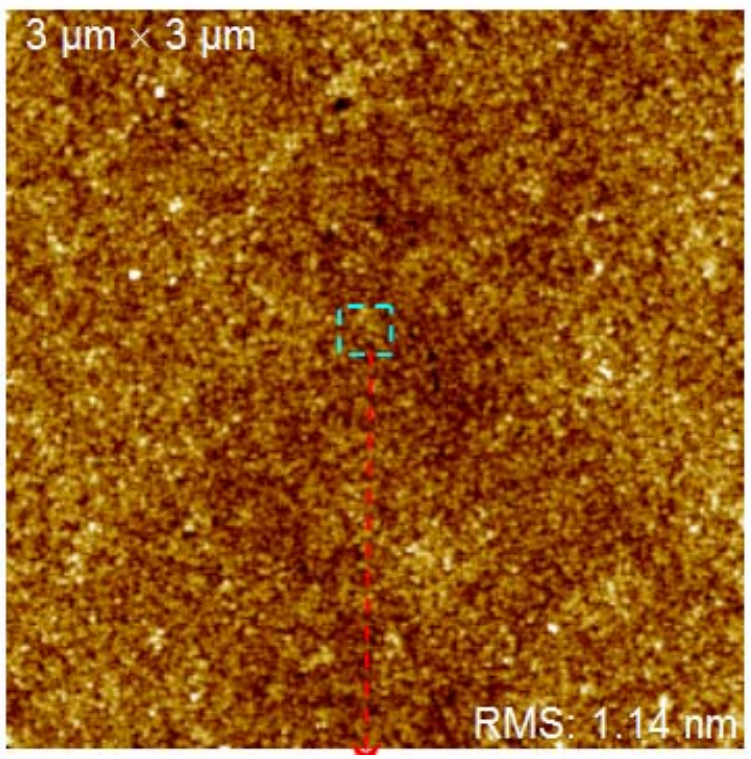

Figure 3.4.10

A change in the deposition flux to rotation velocity ratio is known to have an effect on the morphology of thin films. Glancing Angle Depositions (GLAD) is a technique where a substrate is held at greater than $70^{\circ}$ and used to grow different three dimensional structures based on substrate rotation velocity [24]. Experiments have found that high rotation velocities form more irregular shapes such as helices while low rotation rates form more densely packed columnar structures. 


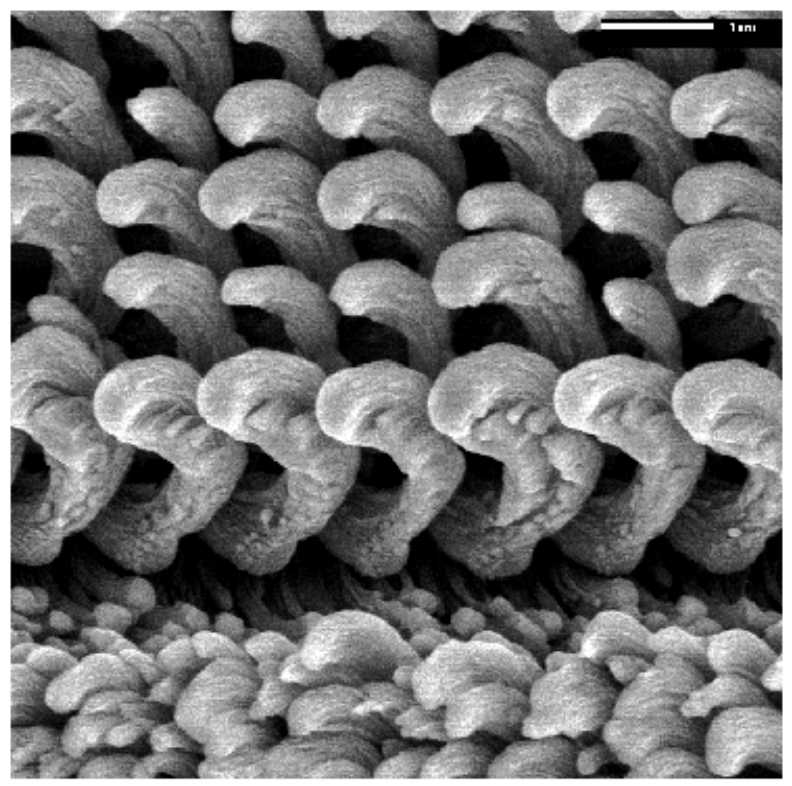

Figure 3.4.11 [25]

Previous depositions occurred at substrate rotations of 50 RPM. To see the effect of substrate rotation on the surface new depositions were performed at 20 RPM while keeping all other parameters the same. AFM images showed a decrease in film surface roughness to $0.63 \mathrm{~nm}$. 


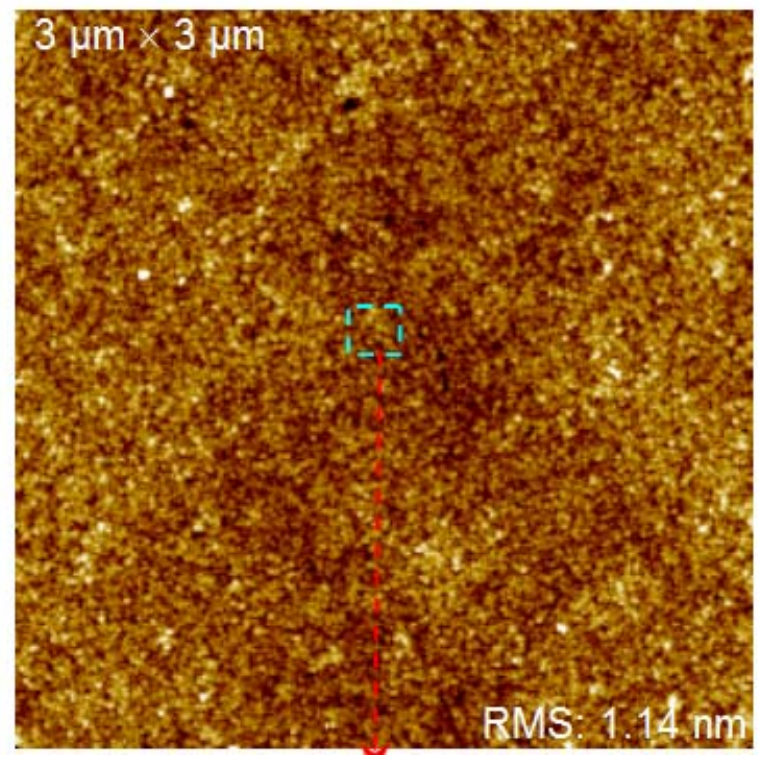

Figure 3.4.12

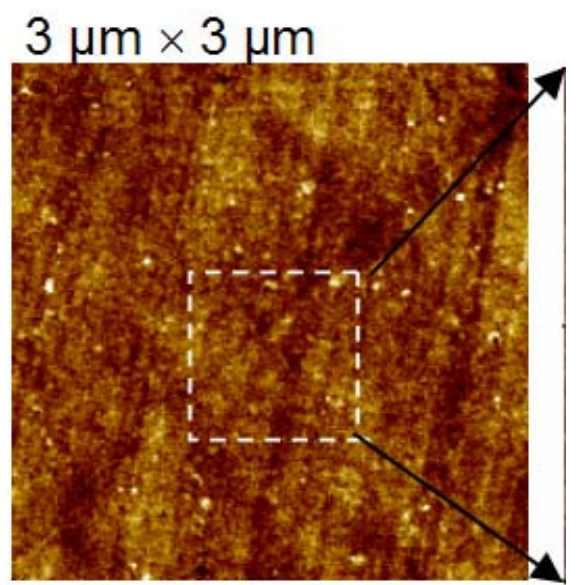

RMS: $0.63 \mathrm{~nm}$

Figure 3.4.13

\section{$\underline{\text { Chapter Four - Conclusion }}$}

\section{- Section 4.1 Improvements}

Many of the issues hindering the growth of acceptable quality films have been overcome but good thickness control over the structure growth remains to be resolved. As more work is done on the growth of the biolayer on the aluminum oxide more work may be possible to optimize the surface morphology and chemistry for biolayer growth.

\section{- Section 4.2}

\section{Improve Thickness Control}

Several options to increase the stability of the quartz crystal monitor are available. Increased cooling from a more powerful water chiller will help alleviate the thermal shock seen by the crystal head when the shutter is opened. Increased thermal transfer between the crystal oscillator and the crystal oscillator housing by using a folded piece of 
gold foil may also help with the thermal instability. If the crystal monitor continues to show instability which prevents control to less than a few nanometers then in-situ sputtering of previously grown films may be possible. This would require an intentional overshoot of the desired thickness for each layer and then removal from the deposition chamber for ellipsometry measurements. Using heavier argon ions the sputtering of a few nanometers of the underlying layer before the next layer of the structure is to be deposited might allow removal of material until the desired thickness is reached.

\section{- Section 4.3}

\section{Decrease Surface Roughness}

Thorough studies of the effect that long term bombardment of oxygen atoms to the films surface remain to be done. Ellipsometer measurements have shown that allowing the ion source to continue bombarding the surface for two to three minutes after the depositions completes does decrease the surface roughness of the films. Performing atomic force microscopy measurements on these samples would provide a more quantitative value for the effect of ion bombardment on surface roughness.

\section{- Section 4.4}

\section{Reactive Depositions of Aluminum Oxide from Aluminum Source}

Thus far studies of the quality of reactively deposited aluminum oxide films have shown only that it is possible to produce a guiding film. As seen with silicon dioxide reactive depositions greater control in thickness and refractive index may be possible through reactive depositions with aluminum source and oxygen ions.

\section{- Section 4.5}

\section{Ion Scrubbing, In-Situ Surface Cleaning}

Due to the mismatch in mass between oxygen atoms and silicon dioxide only a small amount of momentum is transferred when the oxygen ions collide with the 
borofloat glass. Also due to the oxygen ion's highly reactive nature an unknown substance may be forming from contaminates on the substrate surface. If it is found in the future that surface contamination is a problem, the use of argon ions for in-situ substrate cleaning by removing a monolayer from the borofloat wafer would be an option. 


\section{Bibliography}

[1] Turner, Anthony P. F., Biosensors: Past, Present, and Future, Cranfield University, http://www.cranfield.ac.uk/biotech/chinap.htm; (1996)

[2] Shull, J., What is a Biosensors?, University of Maryland Baltimore County, http://userpages.umbc.edu/ j jshull1/ench772/intro

[3] Pathak, S., Resonant Optical Waveguide Biosensor Characterization, Masters Thesis, West Virginia University, (2004)

[4] Lloyd, D., Hornak, L., Pathak,S., Morton, D., Stevenson, I., Application of Ion Beam Assisted Thin Film Deposition Techniques to the Fabrication of a Biosensor Chip With Fieldability Potential for Important Biohazard Detection Applications in Society of Vacuum Coaters, 2004

[5] Mahan, John E., Physical Vapor Deposition of Thin Films, Wiley-Interscience Publishing; (2000)

[6] Boisde, Gilbert and Harmer, Alan, Chemical and biochemical sensing with optical fibers and waveguides, Artech House Publishers ;(1996)

[7] Hadley, G. Ronald, Wide-angle beam propagation using Padé approximant operators, Optics Letters, Volume 17, Issue 20, 1426-1429 October 1992

[8] Mattox, Donald M., Handbook of Physical Vapor Deposition (PVD) Processing, Noyes Publications; (1998)

[9] Smith, Donald L., Thin Film Deposition Principles and Practice, McGraw Hill; (1995)

[10] Palais, Joseph C., Fiber Optic Communications, Prentice-Hall Inc.; (1998)

[11] Zabeida, J.E., Klember-Sapieha, and Martinu L., Ion Bombardment Characteristics During the Growth of Optical Films Using a Cold Cathode Ion Source

[12] Kaufman, Harold R., Robinson, Raymond S., and Seddon, Richard I., End-Hall Ion Source, Journal of Vacuum Science Technology A, Volume 5, Number 4, Pages 20812087; (1987)

[13] Why Ion Assisted Deposition (IAD)?, Denton Vacuum, Moorestown, NJ 08057, http://www.dentonvacuum.com/news/tech.html 
[14] Fourier Transform Infrared Spectroscopy, Ernest Orlando Lawrence Berkeley National Laboratory, http://spectroscopy.lbl.gov/FTIR-Martin/FTIR-

Martin_files/frame.htm

[15] Guide to Using WVASE32, Documentation for M-2000U Spectroscopic Ellipsometer, J. A. Woollam Company Inc., Lincoln, Nebraska 68508

[16] Ellipsometry Tutorial, http://www.jawoollam.com/tutorial_1.html, J. A. Woollam Company Inc., Lincoln, Nebraska 68508

[17] Sun, R., Fouere, J., Moore, R., and Lifshin, E., Principle and Application of Spectroscopic Ellipsometery - Characterization of Films and Coatings for Optical, Structural, Chemical, and Electrical Properties, http://www.sungj.com/publicationList.jsp

[18] Tiwald, Tom, Application Note: Very Thin Films, J.A. Woollam Co. Inc.

[19] M. Yoshimaru, S. Koizumi, and K. Shimokawa, Journal of Vacuum Science and Technology A, 15(6), 2915 (1997).

[20] Ila Prasad and A. N. Chandorkar, Journal of Applied Physics, 94, 2308 (2003)

[21] E. G. Parada, P. González, J. Pou, J. Serra, D. Fernández, B. León, and M. PérezAmor, Journal of Vacuum Science and Technology A, 14(2), 436 (1995)

[22] Morton, Dale E., and Fridman, V., Measurement and Correlation of Ion Beam Current Density to Moisture Stability of Oxide Films Stacks Fabricated by Cold Cathode Ion Assisted Deposition, Denton Vacuum Inc., Moorestown, NJ 08057, http://www.dentonvacuum.com

[23] Morton, Dale E., Blain, J., Hale, J., Optical Monitoring of Thin-films Using Spectroscopic Ellipsometry, Society of Vacuum Coaters, $45^{\text {th }}$ Annual Technical Conference Preceding; (2002)

[24] Robbie, K., and Brett, M.J., Sculptured Thin Films and Glancing Angle Depositions: Growth Mechanics and Applications, Journal of Vacuum Science Technology A, Volume 15, Issue 3, 1460 - 1465; (1997)

[25] Malac, M., and Egerton, R., Observations of the Microscopic Growth Mechanism of Pillar and Helices Formed by Glancing-Angle Thin-Film Deposition, http://laser.phys.ualberta.ca/ mmalac/chapter5.html 